\title{
Electronic structure of Chevrel-phase high-critical-field superconductors
}

\section{Andersen, Ole Krogh; Klose, W.; Nohl, H.}

\section{Published in:}

Physical Review B

Link to article, DOI:

10.1103/PhysRevB.17.1209

Publication date:

1978

Document Version

Publisher's PDF, also known as Version of record

Link back to DTU Orbit

Citation (APA):

Andersen, O. K., Klose, W., \& Nohl, H. (1978). Electronic structure of Chevrel-phase high-critical-field superconductors. Physical Review B, 17(3), 1209-1237. https://doi.org/10.1103/PhysRevB.17.1209

\section{General rights}

Copyright and moral rights for the publications made accessible in the public portal are retained by the authors and/or other copyright owners and it is a condition of accessing publications that users recognise and abide by the legal requirements associated with these rights.

- Users may download and print one copy of any publication from the public portal for the purpose of private study or research.

- You may not further distribute the material or use it for any profit-making activity or commercial gain

- You may freely distribute the URL identifying the publication in the public portal

If you believe that this document breaches copyright please contact us providing details, and we will remove access to the work immediately and investigate your claim 


\title{
Electronic structure of Chevrel-phase high-critical-field superconductors
}

\author{
O. K. Andersen \\ Department of Electrophysics, Technical University, Lyngby, Denmark \\ W. Klose and H. Nohl \\ Kernforschungszentrum Karlsruhe, D75 Karlsruhe, Germany \\ (Received 28 June 1976; revised manuscript received 13 May 1977)
}

\begin{abstract}
Using muffin-tin orbitals and the atomic-sphere approximation, we have studied the band structures of Chevrel-phase molybdenum chalcogenides, $M_{m} M_{6} X_{8-x}$. Generally, these compounds exist for a broad variety of elements, $M=\mathrm{Pb}, \mathrm{Sn}, \mathrm{Ag}, \mathrm{Cu}$ and $X=\mathrm{S}$, Se, Te. $m$ may be between 0 and 2 , depending on the element $M$. We present level schemes, computed for a range of Mo and $X$ potentials, for three $\mathrm{Mo}_{6} X_{14}$ clusters appropriate for the crystal structures of $\mathrm{Mo}_{6} \mathrm{~S}_{8}, \mathrm{Mo}_{6} \mathrm{Se}_{8}$, and $\mathrm{PbMo}_{6} \mathrm{~S}_{7.5}$, respectively. Selfconsistent Mo and $X$ potentials have been estimated. The cluster levels give the positions of the Mo $4 d$-like bands, while the widths and dispersions are estimated analytically in the tight-binding approximation taking the covalent mixing with the $X p$ states into account. The $30 \mathrm{Mo} d$ bands are grouped into narrow subbands derived from the levels for an isolated $\mathrm{Mo}_{6}$ octahedron. The Fermi level falls in a doubly degenerate $E_{\mathrm{g}}$ band with Mo wave functions of $x^{2}-y^{2}$ character and the $E_{\mathrm{g}}$ bandwidths vary between 65 and $35 \mathrm{mRy}$ in the compounds considered. The $E_{g}$ band is probably crossed by a five times wider, singly degenerate $A_{1 g}$ band of predominantly $3 z^{2}-r^{2}$ character. The $E_{g}$ and $A_{1 g}$ bands are the only ones crossing the Fermi level in the ternaries but, in the binaries, the octahedra are elongated and a 50-35 mRy wide $A_{u}$ band, split off from a triply degenerate $T_{2 u}$ band, furthermore overlaps the $E_{\mathrm{g}}$ band. The susceptibilities measured for $\mathrm{SnMo}_{5} \mathrm{~S}_{6}$ and $\mathrm{PbMo}_{5} \mathrm{~S}_{6}$ are in good agreement with our estimates, $N(0)=11$ states/(spin Mo-atom Ry) and $I_{\mathrm{Mo}}^{\text {Stoner }}=40 \mathrm{mRy}$, of the band density of states and the effective exchange-interaction parameter. From the measured electronic-specific-heat coefficients we deduce the value $\lambda=2.5$ for the electron-phonon enhancement. In accord with experimental phonon spectra we estimate frequencies of 10 and $15 \mathrm{meV}$ for a rocking mode of $\mathrm{Mo}_{6} \mathrm{Se}_{8}$ and $\mathrm{Mo}_{6} \mathrm{~S}_{8}$ units, respectively. For the average electron-phonon matrix element in the Gaspari-Gyorffy and atomic-sphere approximations we find $\left\langle I^{2}\right\rangle=3 \times 10^{-3}$ (Ry/bohr radius) ${ }^{2}$. The magnitude and extreme sensitivity to local environment effects of the spin-orbit coupling in the $E_{\mathrm{g}}$ band offer an explanation for the high critical magnetic fields measured in the ternaries.
\end{abstract}

\section{INTRODUCTION}

The ternary molybdenum chalcogenides with the general formula $M_{m} \mathrm{Mo}_{6} X_{8-x}$, first synthesized by Chevrel et al., ${ }^{1}$ have recently created great interest owing to their extraordinary superconducting properties. ${ }^{2}$ In particular, $\mathrm{Pb}_{0.92} \mathrm{Mo}_{6} \mathrm{~S}_{7.5}$ has a critical field of the order of $500 \mathrm{kG}$ and a transition temperature around $13 \mathrm{~K}$.

The crystal structures are trigonal or triclinic and a characteristic feature, illustrated in Fig. 1, is the arrangement of the six Mo atoms of the primitive cell in a nearly perfect octahedron, ${ }^{1,3}$ that is, near the face centers of a cube with edge length $b$. The $X$ atoms are placed slightly inside the corners of this cube. The lattices are almost ${ }^{4}$ cubic with lattice constants $a$, and the essential distortion of the crystals from cubic symmetry is a rotation $\phi$ of $24^{\circ}-26^{\circ}$ about the threefold [111] axis of the $b$ cube with respect to the lattice. The $\mathrm{Mo}_{6}$ octahedra are rather well separated, the smallest intracluster Mo-Mo distance being nearly as in Mo metal and the smallest intercluster MoMo distance being (10-30)\% larger. The smallest intra- and intercluster Mo- $X$ distances are, how- ever, quite similar. The element $M$ may be a large or a small cation. In the first case $M$ stands for $\mathrm{Pb}, \mathrm{Sn}, \mathrm{Ba}, \mathrm{Ag}$, or a rare-earth ion and $m$ is always close to unity. This large cation is placed at the center of inversion between eight $\mathrm{Mo}_{6} X_{8}$ units and hence at the center of a slightly distorted $X$ cube and far away from the Mo atoms. In the real, nonstoichiometric compounds one of the two $X$ atoms nearest to an $M$ atom, i.e., one of those on the threefold axis, may be missing. The crystal structure of $M \mathrm{Mo}_{6} X_{8}$ could in the large-cation case be viewed as a complex $\mathrm{CsCl}$-type structure with the tilted $\mathrm{Mo}_{6} X_{8}$ unit as one component and the large cation as the other. ${ }^{5}$ When $M$ is a small cation, $m$ can take values between zero and 2 , and the ion usually enters the channel between four $\mathrm{Mo}_{6} X_{8}$ units and therefore has Mo atoms among its near neighbors. Although the presence of the element $M$ may have a stabilizing effect on the structure, the nature of this element appears to be of secondary importance for superconductivity, e.g., through modifying the distance between the $\mathrm{Mo}_{6} X_{8}$ units. This view is supported by the fact that $M$ may even be a magnetic ion, provided its position is far from any Mo atom. Fischer ${ }^{2}$ therefore con- 
cluded that the superconducting properties of these materials are mainly determined by the $\mathrm{Mo}_{6}$ octahedra and their relative positions.

Crystal data for $\mathrm{Mo}_{6} \mathrm{~S}_{8}, \mathrm{Mo}_{6} \mathrm{Se}_{8}, \mathrm{Mo}_{6} \mathrm{Te}_{8}$, $\mathrm{PbMo}_{6} \mathrm{~S}_{7.5}$, and $\mathrm{PbMo}_{6} \mathrm{Se}_{8}$ may be found in Table I.

We have investigated the band structures of the above-mentioned compounds as a first step towards an understanding of their superconductivity,

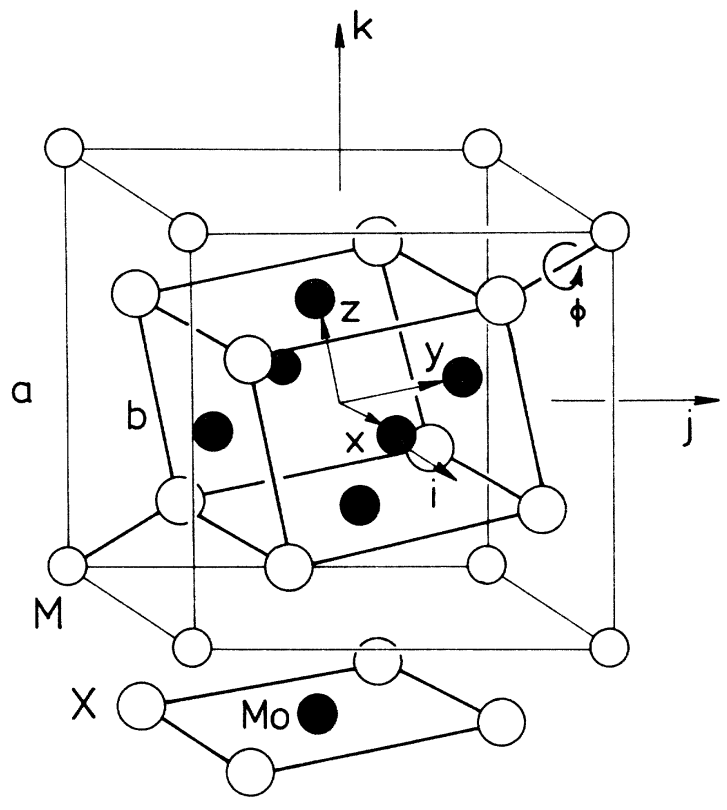

FIG. 1. Primitive cell of $M M_{6} X_{8}$ and top layer of neighboring cell. The coordinate system of the lattice (Ref. 4) (ijk) and that of the basis (xyz) have a common origin at a center of inversion and a common [111] axis along a threefold axis. The two systems are turned by the angle $\phi$ with respect to each other such that the primitive translation vector in the $k$ direction has the $x y z$ coordinates $\frac{1}{3}(1-\cos \phi-\sqrt{3} \sin \phi, 1-\cos \phi+\sqrt{3} \sin \phi$, $1+2 \cos \phi) a$. The $x y z$ system is chosen such that the six Mo atoms have the $x y z$ coordinates $\pm \frac{1}{2}(b, c, c), \pm \frac{1}{2}(c, b, c)$, and $\pm \frac{1}{2}(c, c, b)$, where $c / b \ll 1$. Of the eight $X$ atoms, two are at the threefold axis and six at general positions. Their $x y z$ coordinates are approximately $\frac{1}{2}\left( \pm b^{\prime}, \pm b^{\prime}\right.$, $\left.\pm b^{\prime}\right)$, with $b^{\prime} / b \simeq 1$. For the binary compounds, where the $M$ sites are not occupied, $b^{\prime}$ of the two $X$ atoms at the threefold axis is about $7 \%$ larger than (the average) $b^{\prime}$ of the $X$ atoms at general positions and the Mo octahedron is elongated along the threefold axis. A large cation $M$ will tend to push its nearest $X$ neighbors away and, in the $\mathrm{Pb}$ compounds, $b^{\prime}$ of the $X$ atoms at the threefold axis is $4 \%$ smaller than $b^{\prime}$ of the other six $X$ atoms; occasionally one of the $X$ atoms at the threefold axis may be missing. Values of $a, b, c, \phi$, and a mean value of $b^{\prime}$ for all eight $X$ atoms are given in Table I. The average Mo-Mo and Mo- $X$ intracluster distances are approximately $b / \sqrt{2}$ and $b^{\prime} / \sqrt{2}$. The ratio between these and the smallest Mo-Mo and Mo- $X$ intercluster distances $R$ may be found in the table together with the direction cosines $l$ and $m\left(n^{2}=1-l^{2}-m^{2}\right)$ of the intercluster vectors expressed in the $x y z$ system. and we have therefore concentrated on the Mo $4 d$ like bands near the Fermi level. We first try to answer the question of whether the 30 Mo $d$ bands are separated into a number of narrow bands, derived from the levels for the isolated $\mathrm{Mo}_{6}$ octahedron and broadened by weak intercluster $d-d$ coupling, or the intercluster coupling is strong enough to create a broad, common $d$ band. In Sec. III we compute the Mo $d$ bands, neglecting the mixing with all other bands, and find that the first situation occurs. Simple, analytical expressions for the bands are given in the tight-binding approximation. In Sec. IV we include the hybridization with the Mo $5 s$ states and the covalent mixing with the $X p$ states. The effect on the $4 d$-subband positions is obtained from $\mathrm{Mo}_{6} X_{14}$-cluster calculations, a $\mathrm{Mo}_{6} X_{14}$ cluster being a $\mathrm{Mo}_{6}$ octahedron surrounded by its nearest neighbors. The effect on the subband widths is estimated analytically in the tightbinding approximation and we find that the indirect (Mo $d-X-$ Mo $d$ ) contribution to the intercluster coupling often exceeds the direct (Mo $d$-Mo $d$ ) contribution. From these studies the positions of the Fermi levels may be deduced. Finally our cluster calculations are compared with previous molecular-orbital calculations for $\mathrm{Mo}_{6} X_{8}$ clusters. In Sec. $V$ we try to relate our band structures to physical properties such as susceptibilities, electronic-specific-heat coefficients, rocking modes, superconducting transition temperatures, and critical fields. In Sec. VI we summarize our principal conclusions.

The electronic-structure calculations in the present paper have been performed with the atomicsphere approximation ${ }^{6}$ (ASA) to the Korringa-KohnRostoker (KKR) and linear combinations of muffintin orbitals methods, and these calculations are merely of an explorative nature. For a structure with 15 atoms per cell and open channels, a selfconsistent, non-muffin-tin calculation using the linearized muffin-tin orbital (LMTO) or augmentedplane-wave (LAPW) methods ${ }^{6}$ and a local-density approximation for exchange and correlation is possible but expensive, and we judged that several such calculations for different types of $X$ and $M$ atoms and varying geometries would be needed. We therefore prefer, at this initial stage, to use the LMTO method in the ASA as an accurate model and, taking advantage of its simple, analytical structure, we are able to study entire families of electronic structures, generated from what we feel are reasonable one-electron potentials. Often we illustrate our computational results by simple, analytical estimates. In the following section we begin by summarizing the ASA formalism for clusters and for crystals containing many atoms per primitive cell. 
TABLE I. Crystal data. The experimental results are taken from Ref. 3.

\begin{tabular}{|c|c|c|c|c|c|}
\hline & $\mathrm{Mo}_{6} \mathrm{~S}_{8}$ & $\mathrm{Mo}_{6} \mathrm{Se}_{8}$ & $\mathrm{PbMo}_{6} \mathrm{~S}_{7.5}$ & $\mathrm{PbMo}_{6} \mathrm{Se}_{8}$ & $\mathrm{Mo}_{6} \mathrm{Te}_{8}{ }^{\mathrm{a}}$ \\
\hline \multicolumn{6}{|c|}{ Mo-Mo } \\
\hline$b$ (Bohr radius) & 7.43 & 7.37 & 7.24 & 7.26 & 7.29 \\
\hline$a / b$ & 1.635 & 1.704 & 1.713 & 1.773 & 1.820 \\
\hline$c / b$ & 0.041 & 0.042 & 0.004 & 0.000 & 0.037 \\
\hline$\phi(\mathrm{deg})$ & 25.3 & 24.2 & 25.8 & 25.6 & 24.4 \\
\hline$\sqrt{2} R_{\mathrm{Mo}-\mathrm{Mo}_{0}}^{\text {inter }} / b$ & 1.103 & 1.174 & 1.211 & 1.288 & 1.329 \\
\hline$l_{\text {Mo-Mo }}^{\text {inter }}$ & -0.503 & -0.477 & -0.440 & -0.420 & -0.443 \\
\hline$m_{M 0-M o}^{\text {inter }}$ & 0.532 & 0.494 & 0.564 & 0.549 & 0.482 \\
\hline \multicolumn{6}{|c|}{ Mo- $X$} \\
\hline$b^{\prime} / b$ & 0.87 & 0.92 & 0.90 & 0.95 & 1.00 \\
\hline$\sqrt{2} R_{\mathrm{Mo}-\mathrm{X}}^{\mathrm{inter}} / b^{\prime}$ & 1.01 & 1.03 & 1.05 & 1.05 & 1.05 \\
\hline$l_{\mathrm{Mo}-X}^{\text {inter }}$ & 0.109 & 0.143 & 0.142 & 0.162 & 0.144 \\
\hline$m_{\frac{M}{\text { Mo-X }}}^{\text {inter }}$ & 0.018 & -0.025 & 0.037 & 0.026 & -0.027 \\
\hline
\end{tabular}

${ }^{a}$ R. Chevrel (private communication).

\section{ATOMIC-SPHERE APPROXIMATION}

The one-electron potential in the atomic-sphere approximation (ASA) is spherically symmetric inside spheres surrounding the atoms, and it equals the one-electron energy in the interstitial region. This choice of vanishing kinetic energy outside the spheres greatly simplifies the structure constants of the KKR method for solving Schrödinger's equation, and the associated errors may be substantially reduced by allowing the spheres a slight overlap.

The ASA therefore amounts to substituting the muffin-tin spheres by atomic spheres and to using muffin-tin orbitals (MTO's) $\chi_{t l m}$ with tails decaying like $r^{-l-1} Y_{l m}(\hat{r})$. The condition that the linear combination $\sum_{\overrightarrow{\mathrm{R}} l m} U_{\overrightarrow{\mathrm{R}} l m} \chi_{t l m}(E, \overrightarrow{\mathbf{r}}-\overrightarrow{\mathrm{R}})$ is a solution of Schrödinger's equation at energy $E$ is then that in the neighborhood of any atomic site $\vec{R}^{\prime}$ the sum of the orbitals coming from all other sites $\vec{R}$ should interfere with the orbitals centered at $\vec{R}^{\prime}$ such that the result is a linear combination of the proper solutions $\phi_{t^{\prime} l^{\prime}}\left(E,\left|\overrightarrow{\mathrm{r}}-\overrightarrow{\mathrm{R}}^{\prime}\right|\right) Y_{l^{\prime} m^{\prime}}\left(\left(\overrightarrow{\mathrm{r}}-\overrightarrow{\mathrm{R}}^{\prime}\right) /\left|\overrightarrow{\mathrm{r}}-\overrightarrow{\mathrm{R}}^{\prime}\right|\right)$ of Schrödinger's differential equation for the atomicsphere potential $v_{t},\left(\left|\overrightarrow{\mathbf{r}}-\overrightarrow{\mathbf{R}}^{\prime}\right|\right)$ at that site. This condition gives rise to the set of homogeneous, linear equations $^{7}$

$$
\sum_{\overrightarrow{\mathrm{R}} l m}\left[S_{\overrightarrow{\mathrm{R}}^{\prime} l^{\prime} m^{\prime}, \overrightarrow{\mathrm{R}} l m}-P_{t^{\prime} l^{\prime}}(E) \delta_{\overrightarrow{\mathrm{R}^{\prime}} \overrightarrow{\mathrm{R}}} \delta_{l^{\prime} l} \delta_{m^{\prime} m}\right] U_{\overrightarrow{\mathrm{R}} l m}=0,
$$

where the structure constants $S$ are coefficients for expanding the orbital tails about other sites and where the potential functions $P$ are defined in terms of the radial wave functions and their radial derivatives, evaluated at the atomic-sphere radii $s_{t}$. Specifically,

$$
P_{t l}(E) \equiv 2(2 l+1) \frac{D_{t l}(E)+l+1}{D_{t l}(E)-l}\left(\frac{s}{s_{t}}\right)^{2 l+1},
$$

where $D_{t l}(E) \equiv s_{t} \phi_{t l}^{\prime}\left(E, s_{t}\right) / \phi_{t l}\left(E, s_{t}\right)$ are the logarithmic derivative functions and where $s$ is some linear dimension of the structure.

The KKR equations (1) may be solved at those energies $E_{j}$ for which the determinant of the matrix in the square bracket vanishes. These are then the one-electron energies and, in the neighborhood of any site $\vec{R}^{\prime}$, the corresponding wave functions are given by

$$
\begin{gathered}
\psi_{j}(\overrightarrow{\mathrm{r}})=\sum_{l^{\prime} m^{\prime}} U_{\overrightarrow{\mathrm{R}}^{\prime} l^{\prime} m^{\prime}, j}\left[\dot{P}_{t^{\prime} l^{\prime}}\left(E_{j}\right)\right]^{1 / 2} \phi_{t^{\prime} l^{\prime}}\left(E_{j},\left|\overrightarrow{\mathrm{r}}-\overrightarrow{\mathrm{R}}^{\prime}\right|\right) \\
\times Y_{l^{\prime} m^{\prime}}\left(\left(\overrightarrow{\mathrm{r}}-\overrightarrow{\mathrm{R}}^{\prime}\right) /\left|\overrightarrow{\mathrm{r}}-\overrightarrow{\mathrm{R}}^{\prime}\right|\right),
\end{gathered}
$$

where $\phi Y$ snould be normalized to unity in the sphere at $\vec{R}^{\prime}$. The potential functions are never decreasing functions of energy and the appearance of the energy derivatives $\dot{P}$ as normalization factors in (3) is connected with the fact that, in the neighborhood of any one-electron energy, we may write $P_{t l}(E)=P_{t l}\left(E_{j}\right)+\left(E-E_{j}\right) \dot{P}_{t l}\left(E_{j}\right)$ and then regard (1) as eigenvalue equations. The ratio between the probabilities that the electron is in a sphere of type $t$ and has angular momentum $l$ and that it is in a sphere of type $t^{\prime}$ and has angular momentum $l^{\prime}$ is 


$$
\left[\dot{P}_{t l}\left(E_{j}\right) U_{t l, j}^{2}\right] /\left[\dot{P}_{t^{\prime} l^{\prime}}\left(E_{j}\right) U_{t^{\prime} l^{\prime}, j}^{2}\right]
$$

where

$$
U_{t l, j}^{2} \equiv \sum_{\overrightarrow{\mathrm{R}}}^{h(t)} \sum_{m}^{2 l+1}\left|U_{\overrightarrow{\mathrm{R}} l m, j}\right|^{2}
$$

and the $\vec{R}$ sum runs over the $h(t)$ sites occupied by atoms of type $t$.

The structure constants in the ASA are canonical in the sense that they depend neither on the energy, on the atomic-sphere radii, nor on the scale of the structure. They are tabulated in Table II as functions of the distance $R$ and the direction cosines $(l, m, n)$ of the interatomic vector $\vec{R}-\vec{R}^{\prime}$.

\section{A. Canonical theory}

In studies like the present, where the choice of atomic-sphere radii and potentials is not obvious a priori, the ASA is a most convenient model because it completely separates the potential and structure dependences of the one-electron energies and wave functions. We may even solve (1) without specifying the potential functions but regarding them, rather than the energy, as the independent variables. The surfaces in $\vec{P}$ space (see Fig. 10) for which the determinant of the matrix in the square bracket of Eq. (1) vanishes depend only on the structure; they are just another representation of the structure constants. We shall now show that, for a given point $\overrightarrow{\mathrm{P}}_{j}$ on the $j$ th surface, the canonical probabilities $U_{t l, j}^{2}$ form a vector $\overrightarrow{\mathrm{U}}_{j}^{2}$ which is normal to the surface at that point.

We define the function

$$
T(\overrightarrow{\mathrm{U}}, \overrightarrow{\mathrm{P}}) \equiv \sum_{L} \sum_{L^{\prime}} U_{L}^{*}\left(S_{L L^{\prime}}-P_{L} \delta_{L L^{\prime}}\right) U_{L^{\prime}}
$$

of the independent vector variables $\vec{P}$ and $\vec{U}$ with the components $P_{L} \equiv P_{\overrightarrow{\mathrm{R}} \imath m}$ and $U_{L} \equiv U_{\overrightarrow{\mathrm{R}} \imath m}$. Of these variables, $\vec{P}$ is real and $\vec{U}$ is complex. The total differential of $T$ is

TABLE II. Canonical structure constants. The notation follows that of Slater and Koster (Ref. 10). The vector from the first to the second orbital has the length $R$ and the direction cosines $l, m$, and $n$. The distance $s$, which also enters the definition of the potential functions, is arbitrary. The entries not given in the table may be found by cyclically permuting the coordinates and direction cosines. Moreover, $S\left(l^{\prime} m^{\prime}, l m\right)=(-)^{l \cdot+l} S\left(l m, l^{\prime} m^{\prime}\right)$, where $l m$ refers to the angular momentum. The present real structure constants equal those defined in Ref. 6 [(4.47)] times $i^{l^{l-l}}\left(s / s_{t}\right)^{l^{\prime}+1 / 2}\left(s / s_{t}\right)^{l+1 / 2}$.

\begin{tabular}{ll}
\hline \hline$S(s, s)$ & $-2(s / R)$ \\
$S(s, x)$ & $l \times 2 \sqrt{3}(s / R)^{2}$ \\
$S(x, x)$ & $\left(3 l^{2}-1\right) \times 6(s / R)^{3}$ \\
$S(x, y)$ & $3 l m \times 6(s / R)^{3}$ \\
$S(s, x y)$ & $-\sqrt{3} l m \times 2 \sqrt{5}(s / R)^{3}$ \\
$S\left(s, x^{2}-y^{2}\right)$ & $-\sqrt{3}\left(l^{2}-m^{2}\right) / 2 \times 2 \sqrt{5}(s / R)^{3}$ \\
$S\left(s, 3 z^{2}-r^{2}\right)$ & $\left(1-3 n^{2}\right) / 2 \times 2 \sqrt{5}(s / R)^{3}$ \\
$S(x, x y)$ & $\left(1-5 l^{2}\right) m \times 6 \sqrt{5}(s / R)^{4}$ \\
$S\left(x, x^{2}-y^{2}\right)$ & {$\left[1-\frac{5}{2}\left(l^{2}-m^{2}\right)\right] l \times 6 \sqrt{5}(s / R)^{4}$} \\
$S(x, y z)$ & $-5 l m n \times 6 \sqrt{5}(s / R)^{4}$ \\
$S\left(z, x^{2}-y^{2}\right)$ & $-5 n\left(l^{2}-m^{2}\right) / 2 \times 6 \sqrt{5}(s / R)^{4}$ \\
$S\left(x, 3 z^{2}-r^{2}\right)$ & $\left(\frac{1}{2} \sqrt{3}\right)\left(1-5 n^{2}\right) l \times 6 \sqrt{5}(s / R)^{4}$ \\
$S\left(z, 3 z^{2}-r^{2}\right)$ & $\left(\frac{1}{2} \sqrt{3}\right)\left(3-5 n^{2}\right) n \times 6 \sqrt{5}(s / R)^{4}$ \\
$S(x y, x y)$ & $\left(-35 l^{2} m^{2}-5 n^{2}+4\right) \times 10(s / R)^{5}$ \\
$S\left(x^{2}-y^{2}, x^{2}-y^{2}\right)$ & {$\left[-\frac{35}{4}\left(l^{2}-m^{2}\right)^{2}-5 n^{2}+4\right] \times 10(s / R)^{5}$} \\
$S\left(3 z^{2}-r^{2}, 3 z^{2}-r^{2}\right)$ & $\left(-\frac{3}{4}\right)\left(35 n^{4}-30 n^{2}+3\right) \times 10(s / R)^{5}$ \\
$S\left(x y, x^{2}-y^{2}\right)$ & $-35 l m\left(l^{2}-m^{2}\right) / 2 \times 10(s / R)^{5}$ \\
$S\left(z x, x^{2}-y^{2}\right)$ & $-5\left[\frac{7}{2}\left(l^{2}-m^{2}\right)-1\right] l n \times 10(s / R)^{5}$ \\
$S\left(y z, x^{2}-y^{2}\right)$ & $5\left[\frac{7}{2}\left(m^{2}-l^{2}\right)-1\right] m n \times 10(s / R)^{5}$ \\
$S(y z, z x)$ & $-5\left(7 n^{2}-1\right) l m \times 10(s / R)^{5}$ \\
$S\left(x^{2}-y^{2}, 3 z^{2}-r^{2}\right)$ & $\left(-\frac{1}{2} \sqrt{3}\right) 5\left(7 n^{2}-1\right)\left(l^{2}-m^{2}\right) / 2 \times 10(s / R)^{5}$ \\
$S\left(x y, 3 z^{2}-r^{2}\right)$ & $\left(-\frac{1}{2} \sqrt{3}\right) 5\left(7 n^{2}-1\right) l m \times 10(s / R)^{5}$ \\
\hline \hline
\end{tabular}




$$
\begin{aligned}
d T & =\sum_{L} \sum_{L^{\prime}} d U_{L}^{*}\left(S_{L L^{\prime}}-P_{L} \delta_{L L^{\prime}}\right) U_{L^{\prime}}+\text { c.c. } \\
& -\sum_{L} d P_{L}\left|U_{L}\right|^{2}
\end{aligned}
$$

because the structure matrix is Hermitian. For any point $\overrightarrow{\mathbf{P}}_{j}$ on the $j$ th canonical eigenvalue surface and for the corresponding wave function coefficient $\vec{U}_{j}$, it is true that

$$
\sum_{L^{\prime}}\left(S_{L L^{\prime}}-P_{L, j} \delta_{L L^{\prime}}\right) U_{L^{\prime}, j}=0
$$

for all values of $L$. Consequently, $T\left(\vec{U}_{j}, \overrightarrow{\mathrm{P}}_{j}\right) \equiv 0$ and $d T\left(\overrightarrow{\mathrm{U}}_{j}, \overrightarrow{\mathbf{P}}_{j}\right)=0$ when $\overrightarrow{\mathbf{P}}_{j}$ is displaced arbitrarily along the surface by $d \overrightarrow{\mathbf{P}}_{j}$. From the expression for the total differential of $T$, therefore, $\sum_{L} d P_{L, j}\left|U_{L, j}\right|^{2}$ $=0$. If we then restrict the components of $\overrightarrow{\mathrm{P}}_{j}$ corresponding to the same type of sphere $t$ and magnitude of angular momentum $l$ to be equal, we may sum over $\overrightarrow{\mathrm{R}}_{t}$ and $m$ and thus arrive at $d \overrightarrow{\mathrm{P}}_{j} \cdot \overrightarrow{\mathrm{U}}_{j}^{2}=0$; i.e., $\overrightarrow{\mathrm{U}}_{j}^{2}$ is normal to the canonical eigenvalue surface.

A given potential specifies a path $\overrightarrow{\mathrm{P}}(E)$, and a corresponding one-electron energy $E_{j}$ is the value of the parameter $E$ along the path at its intersection with the $j$ th canonical eigenvalue surface, which we shall now assume is given by

$$
f_{j}(\overrightarrow{\mathbf{P}})=0 \text {. }
$$

The probability that the $j$ electron is in a sphere of type $t$ and has angular momentum $l$ about its center may then be expressed as

$$
\dot{P}_{t l}\left(E_{j}\right) U_{t l, j}^{2}=\frac{\dot{P}_{t l}\left(E_{j}\right) f_{t l_{, j},}^{\prime}\left(\overrightarrow{\mathrm{P}}\left(E_{j}\right)\right)}{\sum_{t^{\prime} l^{\prime}} \dot{P}_{t^{\prime} l^{\prime}}\left(E_{j}\right) f_{t^{\prime} l^{\prime}, j}^{\prime}\left(\overrightarrow{\mathrm{P}}\left(E_{j}\right)\right)},
$$

where $f_{t l, j}^{\prime} \equiv \partial f_{j} / \partial P_{t l}$ and where, in performing the normalization, we have neglected the probability that the electron is in the interstitial region, or, in other words, we have "filled space with spheres." If the division of space into (slightly overlapping) spheres is made judiciously, this approximation is a good one. In any case, the choice of sphere radii does not influence the canonical eigenvalue surfaces but only the potential functions, and the ratio between the $t l$ and $t^{\prime} l^{\prime}$ probabilities is independent of the probability for the interstitial region. The canonical eigenvalue surfaces thus contain all the structural information needed to construct the electron density and hence to perform a self-consistent calculation within the atomic-sphere and local-density approximations. Once these surfaces have been determined there are no further eigenvalue problems to be solved for that structure.

For crystals, ${ }^{7}$ also the Brillouin-zone sums can be done once and for all and, in that case, the eigenvalue surfaces are substituted by a continuous and differentiable number-of-states function $n(\overrightarrow{\mathrm{P}})$, in terms of which the projected densities of states are

$$
N_{t \imath}(E)=\dot{P}_{t \imath}(E) n_{t \imath}^{\prime}(\overrightarrow{\mathrm{P}}(E)),
$$

where $n_{t l}^{\prime} \equiv \partial n / \partial P_{t l}$. Equation (7a) may be proved in the following way: For a crystal, the one-electron energy for band $j$ and Bloch vector $\overrightarrow{\mathrm{k}}$ is $E_{j}(\overrightarrow{\mathrm{k}})$, the $t l$ probability is $\dot{P}_{t l}\left(E_{j}(\overrightarrow{\mathrm{k}})\right) U_{t l, j}^{2}(\overrightarrow{\mathrm{k}})$, and the corresponding projected density of states is defined as

$$
N_{t l}(E) \equiv \sum_{j} V_{\mathrm{Bz}}^{-1} \int d^{3} k \delta\left(E-E_{j}(\overrightarrow{\mathrm{k}})\right) \dot{P}_{t l}(E) U_{t l_{\mathrm{l}} j}^{2}(\overrightarrow{\mathrm{k}}),
$$

where $V_{\mathrm{BZ}}=(2 \pi)^{3} / \Omega$ is the Brillouin-zone volume. If the canonical eigenvalue surface for band $j$ and Bloch vector $\vec{k}$ is given by $f_{j}(\vec{k}, \overrightarrow{\mathbf{p}})=0$, the oneelectron energy is the solution of $f_{j}\left(\overrightarrow{\mathrm{k}}, \overrightarrow{\mathrm{p}}\left(E_{j}(\overrightarrow{\mathrm{k}})\right)\right)=0$, and consequently

$$
\begin{aligned}
\delta\left(E-E_{j}(\overrightarrow{\mathrm{k}})\right)= & \delta\left(f_{j}(\overrightarrow{\mathrm{k}}, \overrightarrow{\mathrm{P}}(E))\right) d f_{j} / d E \\
= & \delta\left(f_{j}(\overrightarrow{\mathrm{k}}, \overrightarrow{\mathrm{P}}(E))\right) \\
& \times \sum_{t^{\prime} l^{\prime}} \dot{P}_{t^{\prime} l^{\prime}}(E) f_{t^{\prime} l^{\prime}, j}^{\prime}(\overrightarrow{\mathrm{k}}, \overrightarrow{\mathrm{P}}(E)) .
\end{aligned}
$$

The $t l$ probability may be expressed as in (6) and, as a result, we may write $(7 \mathrm{~b})$ as

$$
\begin{aligned}
N_{t l}(E)= & \dot{P}_{t l}(E) \sum_{j} V_{\mathrm{BZ}}^{-1} \\
& \times \int d^{3} k \delta\left(f_{j}(\overrightarrow{\mathrm{k}}, \overrightarrow{\mathrm{P}}(E))\right) f_{t l, j}^{\prime}(\overrightarrow{\mathrm{k}}, \overrightarrow{\mathrm{P}}(E)) .
\end{aligned}
$$

This shows that

$$
d n(\overrightarrow{\mathbf{P}})=\sum_{j} V_{\mathrm{BZ}}^{-1} \int d^{3} k \delta\left(f_{j}(\overrightarrow{\mathrm{k}}, \overrightarrow{\mathrm{P}})\right) d f_{j}(\overrightarrow{\mathrm{k}}, \overrightarrow{\mathrm{P}})
$$

is a total differential with respect to $\overrightarrow{\mathrm{P}}$; Q.E.D. The total density of states is, of course,

$$
N(E)=\sum_{t l} N_{t l}(E)=\sum_{j} V_{\mathrm{BZ}}^{-1} \int d^{3} k \delta\left(E-E_{j}(\overrightarrow{\mathrm{k}})\right) .
$$

One might summarize the canonical theory by saying that the atomic positions define a numberof-states mountain (actually only one face of a mountain) in $\overrightarrow{\mathrm{P}}$ space. The potentials and sizes of the atomic spheres merely specify a path $\overrightarrow{\mathrm{P}}(E)$ up this mountain, with $E$ playing the role of the time along the path. At a given point of a path, the power $(\partial n / \partial \overrightarrow{\mathbf{P}}) \cdot(d \overrightarrow{\mathbf{P}} / d E)$ of the force $\partial n / \partial \overrightarrow{\mathbf{P}}$ is the total density of states, while the power of the force component $\partial n / \partial P_{t l}$ is the $t l$-projected density of states. For a finite system the mountain is terraced and for crystals it is smooth. 


\section{B. Potential functions}

Useful parametrizations of the potential functions are

$$
\begin{aligned}
P_{t l}(E) & =\frac{\left(E-C_{t l}\right) \mu_{t l} s_{t}^{2}}{1+\gamma_{t l}\left(E-C_{t l}\right) \mu_{t l} s_{t}^{2}}\left(\frac{s}{s_{t}}\right)^{2 l+1} \\
& =\left(\frac{2(2 l+1)^{2}(2 l+3)}{\left(V_{t l}-E\right) \tau_{t l} s_{t}^{2}}+\frac{1}{\gamma_{t l}}\right)\left(\frac{s}{s_{t}}\right)^{2 l+1},
\end{aligned}
$$

valid for an energy range of order $0.5 \mathrm{Ry}$. Here, $C_{t l}$ is called the center of the $t l$ band, $V_{t l}$ is the square-well pseudopotential, $\mu_{t l}$ and $\tau_{t l}$ are intrinsic band masses relative to that of free electrons, and $\gamma_{t l}$ is a distortion parameter. ${ }^{8}$ The potential parameters for some 40 elemental metals have been listed in Ref. 6 .

For the molybdenum chalcogenides we choose the dimension $s$ entering the definition of the structure constants and the potential functions to be (3/ $16 \pi)^{1 / 3} b$, which is the atomic-sphere radius in the corresponding fcc structure.

In Table III we give values of the potential parameters for Mo and $X$, appropriate for energies in the occupied part of the Mo $d$ band. These approximate values were estimated from atomicsphere potentials constructed from renormalized atomic charge densities and using the HedinLundqvist approximation ${ }^{9}$ for exchange and correlation. The amount of renormalized charge, and hence $C_{\mathrm{Mo} l}-C_{X l^{\prime}}$, was determined self-consistently using the canonical eigenvalue surfaces shown in Figs. 9 and 10, and the results are explained in Sec. IV B. The effect of charge transfer on the other potential parameters was neglected. For $\mathrm{PbMo}_{6} \mathrm{~S}_{7.5}$ the sphere radii $s_{t}$ were chosen to be
$14 \%$ larger than those of touching spheres, whereby the Mo radius takes the same value as the Wigner-Seitz radius in bcc Mo metal. This same Mo radius was then used for all other compounds and the chalcogen radii were determined by the requirement of $14 \%$ overlap; i.e., $s_{X}=(1.14 / \sqrt{2}) b^{\prime}$ $-s_{\text {Mo }}$ (See Fig. 1.) The radii thus determined agree within $10 \%$ with those $l$-dependent atomic radii for which the logarithmic derivative of the isolated-atom valence orbital (i.e., Mo $s$, Mo $d$, and $X p)$ takes the value $-(l+1)$.

The Mo and $\mathrm{S}$ potential functions for $\mathrm{PbMo}_{6} \mathrm{~S}_{7.5}$ are shown in Fig. 2.

\section{Equivalence with the two-center approximation}

In an energy range so narrow that the potential path is a straight line, i.e., that

$$
P_{t l}(E)=\left(E-\hat{C}_{t l}\right) / \Delta_{t l},
$$

the ASA is mathematically equivalent with the twocenter approximation ${ }^{10}$ to the linear combination of atomic orbitals (LCAO) method. The effective overlap matrix is the unit matrix, while the effective-Hamiltonian matrix is

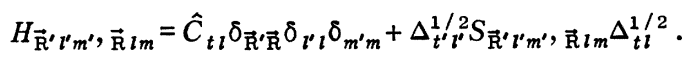

Consequently, $\hat{C}_{t l}$ is the center of gravity of the unhybridized $t l$ energies and $\Delta^{1 / 2} S \Delta^{1 / 2}$ are the effective transfer integrals. As seen from Table II, these integrals are far simpler than those encountered in the LCAO method. The effective twocenter integrals are in fact

TABLE III. Approximate self-consistent potential parameters. $s_{X} \equiv(1.14 / \sqrt{2}) b^{\prime}-s_{\text {Mo }}$. Radii $s$ are in Bohr radii. Energies $C, \hat{C}$, and $\Delta$ are in Ry. Central energies $C$ and $\hat{C}$ are relative to $C_{\text {Mo } d} \cdot E_{\nu} \approx C_{\text {Mo } d}$ and $C_{X p}$ for Mo and $X$ potentials, respectively.

\begin{tabular}{lcccccc}
\hline \hline & $\begin{array}{c}\text { Mo potential } \\
\text { (all compounds) }\end{array}$ & $\mathrm{Mo}_{6} \mathrm{~S}_{8}$ & $\mathrm{Mo}_{6} \mathrm{Se}_{8}$ & $\mathrm{PbMo}_{6} \mathrm{~S}_{7.5}$ & $\mathrm{PbMo}_{6} \mathrm{Se}_{8}$ & $\mathrm{Mo}_{6} \mathrm{Te}_{8}$ \\
\hline$C_{s}$ & -0.11 & -1.7 & -1.9 & -1.8 & -2.1 & -1.7 \\
$C_{p}$ & 0.89 & -0.35 & -0.31 & -0.32 & -0.29 & -0.25 \\
$C_{d}$ & 0.00 & 1.8 & 1.7 & 1.6 & 1.4 & 1.3 \\
$\mu_{s}$ & 0.66 & 0.9 & 0.6 & 0.7 & 0.3 & 0.4 \\
$\mu_{p}$ & 0.76 & 1.4 & 1.3 & 1.5 & 1.4 & 1.2 \\
$\mu_{d}$ & 4.0 & 1.2 & 0.9 & 1.3 & 1.0 & 0.8 \\
$\gamma_{s}$ & 0.42 & 0.34 & 0.29 & 0.30 & 0.24 & 0.30 \\
$\gamma_{p}$ & 0.12 & 0.08 & 0.08 & 0.08 & 0.08 & 0.08 \\
$\gamma_{d}$ & 0.00 & 0.05 & 0.06 & 0.05 & 0.05 & 0.06 \\
$\hat{C}_{p}\left(C_{\text {Mo d }}\right)$ & & -0.42 & -0.38 & -0.39 & -0.36 & -0.30 \\
$\Delta_{p}\left(C_{\text {Mo d }}\right)$ & & 0.09 & 0.12 & 0.10 & 0.12 & 0.15 \\
$\Delta_{p}\left(C_{X p}\right)$ & & 0.065 & 0.081 & 0.069 & 0.082 & 0.11 \\
$\Delta_{d}\left(C_{\text {Mo d }}\right)$ & $\approx 0.035$ & & & & & \\
$s_{t}$ & 2.92 & 2.29 & 2.55 & 2.33 & 2.64 & 2.96 \\
$s=0.3908 b$ & & 2.90 & 2.88 & 2.83 & 2.84 & 2.85 \\
\hline \hline
\end{tabular}




$$
\left(l^{\prime} l M\right)_{t^{\prime} t}=(-)^{l+M+1}\left(l^{\prime}+l\right) ! 2\left(\frac{\left(2 l^{\prime}+1\right)(2 l+1) \Delta_{t^{\prime} l^{\prime} \Delta_{t l}}}{\left(l^{\prime}+M\right) !\left(l^{\prime}-M\right) !(l+M) !(l-M) !}\right)^{1 / 2}\left(\frac{s}{R}\right)^{l^{\prime}+l+1}
$$

For example,

$$
\begin{aligned}
& (d d \sigma)=-6) \\
& (d d \pi)=4 \quad \times 10(s / R)^{5} \Delta_{d}, \\
& (d d \delta)=-1) \\
& (p d \sigma)=-\sqrt{3} \\
& (p d \pi)=1\} \times 6 \sqrt{5}(s / R)^{4} \sqrt{\Delta_{p} \Delta_{d}},
\end{aligned}
$$

where $p, d$ stand for $l, l^{\prime}=1,2$ and $\sigma, \pi, \delta$ for $M$ $=0,1,2$.

It should be noted that the interaction (15) has long range for $l^{\prime}+l \lesssim 3$; the $s-s$ interaction, in particular, falls off as $1 / R$. For accurate calculations on finite systems the interstitial region must therefore be bound by embedding the cluster in a Watson sphere, outside which the tails of the muffin-tin orbitals are then augmented by energy-

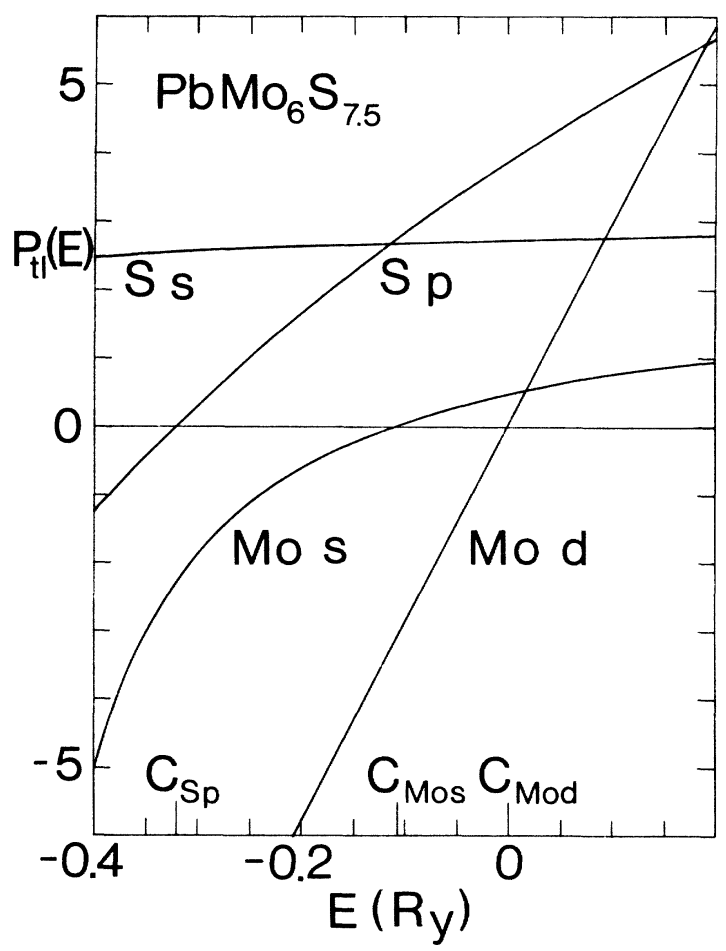

FIG. 2. Potential functions for molybdenum and sulphur spheres in $\mathrm{PbMo}_{6} \mathrm{~S}_{7.5}$ as given by Eq. (11) with the potential parameters in Table III. The zero of energy is at $C_{\text {Mod }}$. The energy where $P_{\text {Mos } s}$ diverges, and which is at the bottom of the $s$ band in Mo metal, is $V_{\text {Mos }}=-0.53$ Ry. derivative functions in the usual way. ${ }^{6}$ For crystals the lattice summations must be performed by the Ewald method. In monatomic crystals $S_{s, s}(\overrightarrow{\mathrm{k}})--6(k s)^{-2}$ for $k \rightarrow 0$ and, using (12) with $s=s_{t}$ $=s_{\text {ws }}$, we realize that $V_{\mathrm{s}}$ is the bottom of the $s$ band.

\section{Pure levels and weak hybridization}

The average of the $(2 l+1) h(t) \equiv h(t l)$ eigenvalues $S_{t l, j}$ of the $t l$ diagonal block of the structure constants is zero, because the structure constants referring to the same site $\left(\vec{R}=\vec{R}^{\prime}\right)$ vanish, and a measure of the range covered by the $t l$ eigenvalues is

$$
\begin{aligned}
& \left(12 h(t l)^{-1} \sum_{j}^{h(t l)} S_{t l, j}^{2}\right)^{1 / 2} \\
& =\left(12 h(t l)^{-1} \sum_{\overrightarrow{\mathrm{R}}^{\prime} m^{\prime}}^{h(t l)} \sum_{\overrightarrow{\mathrm{R}} m}^{h(t l)}\left|S_{\overrightarrow{\mathrm{R}}^{\prime} l m^{\prime}, \overrightarrow{\mathrm{R}} l m}\right|^{2}\right)^{1 / 2} \equiv W_{t l},
\end{aligned}
$$

where the trace of the $t l$ diagonal block squared is given by (22) below with $t=t^{\prime}$ and $l=l^{\prime}$. If (13) holds over this range, the extent of the unhybridized $t l$ energies is $W_{t l} \Delta_{t l}$.

If we include hybridization, under the assumption that it is weak, i.e., that $\left|P_{t^{\prime} l^{\prime}}\right| \gg W_{t^{\prime} l^{\prime}}$ for all $t^{\prime} l^{\prime} \neq t l$, the $t l$-like canonical eigenvalue surfaces are given by

$$
f_{j}(\overrightarrow{\mathrm{P}}) \equiv P_{t l}-S_{t l, j}-\sum_{t^{\prime} l^{\prime}}^{\prime}\left|S_{\left.t^{\prime} l^{\prime}\right|_{j}}^{t}\right|_{j}^{2} P_{t^{\prime} l^{\prime}}^{-1}=0
$$

as obtained by folding down the linear equations (1), and with

$$
\left|S_{t^{\prime} l^{\prime}}^{t l}\right|_{j}^{2} \equiv \sum_{\vec{R}^{\prime} m^{\prime}}^{h\left(t^{\prime} l^{\prime}\right)} \sum_{\overrightarrow{\mathrm{R}} m}^{h(t l)}\left|S_{\overrightarrow{\mathrm{R}}^{\prime} l^{\prime} m^{\prime}, \overrightarrow{\mathrm{R}} l m} \tilde{U}_{\overrightarrow{\mathrm{R}} l m, j}\right|^{2} .
$$

Here, the eigenvector of the $t l$ diagonal block of the structure constants is assumed to be normalized according to $\tilde{U}_{t l, j}^{2}=1$, and the terms $\sum_{t^{\prime} l^{\prime}}^{\prime}\left|S_{t^{\prime} l^{\prime}}^{t l}\right|_{j}^{2} P_{t^{\prime} l^{\prime}}^{-1}$ in (17a) have the form of crystalfield terms.

Using (6) together with (17a), the probability that the $t l$-like $j$ electron is in a sphere of type $t^{\prime}$ and has angular momentum $l^{\prime}$ about its center is

$$
\dot{P}_{t^{\prime} l^{\prime}}\left(E_{j}\right) U_{t^{\prime} l^{\prime}, j}^{2}=p_{t^{\prime} l^{\prime}, j}^{t l}\left(1+\sum_{t^{\prime \prime} l^{\prime \prime}}^{\prime} p_{t^{\prime \prime} l^{\prime \prime}, j}^{t l}\right)^{-1},
$$

where the prime indicates that the term with $t^{\prime \prime} l^{\prime \prime}$ $=t l$ is absent and where

$$
p_{t^{\prime} l^{\prime}, j}^{t l}=\left.\frac{d\left(-P_{t^{\prime} l^{\prime}}^{-1}\right) / d E}{d P_{t l} / d E}\right|_{E_{j}}\left|S_{t^{\prime} l^{\prime}}^{t l}\right|_{j}^{2}
$$


is the ratio between the $t^{\prime} l^{\prime}$ and $t l$ probabilities (i.e., $p_{t l, j}^{t l} \equiv 1$ ). In terms of the potential parameters (11)

$$
\begin{aligned}
p_{t^{\prime} l^{\prime}, j}^{t l} & =\frac{\Delta_{t^{\prime} l^{\prime}}\left(C_{t^{\prime} l^{\prime}}\right) \Delta_{t l}\left(E_{j}\right)}{\left(C_{t^{\prime} l^{\prime}}-E_{j}\right)^{2}}\left|S_{t^{\prime} l^{\prime}}^{t l}\right|_{j}^{2} \\
& \approx \frac{\Delta_{t^{\prime} l^{\prime}} \Delta_{t l}}{\left(C_{t^{\prime} l^{\prime}}-C_{t l}\right)^{2}}\left|S_{t^{\prime} l^{\prime}}^{t l}\right|_{j}^{2},
\end{aligned}
$$

where, in the last approximation, we have used $E_{j} \approx C_{t l}$ and both $\Delta$ 's therefore refer to their respective band centers, i.e., $\Delta=\left(\mu s_{t}^{2}\right)^{-1}\left(s_{t} / s\right)^{2 l+1}$. For a crystal ${ }^{7}$

$$
f_{j}(\overrightarrow{\mathrm{k}}, \overrightarrow{\mathrm{P}})=P_{t l}-S_{t l, j}(\overrightarrow{\mathrm{k}})-\sum_{t^{\prime} l^{\prime}}^{\prime}\left|S_{t^{\prime} l^{\prime}}^{t l}(\overrightarrow{\mathrm{k}})\right|_{j}^{2} P_{t^{\prime} l^{\prime}}^{-1},
$$

with

$$
\left|S_{t^{\prime} l^{\prime}}^{t l}(\overrightarrow{\mathrm{k}})\right|_{j}^{2} \equiv \sum_{\overrightarrow{\mathrm{R}}^{\prime} m^{\prime}}^{h\left(t^{\prime} l^{\prime}\right)} \sum_{\overrightarrow{\mathrm{R}} m}^{h(t l)}\left|S_{\overrightarrow{\mathrm{R}}^{\prime} l^{\prime} m^{\prime}, R l m}(\overrightarrow{\mathrm{k}}) \tilde{U}_{\overrightarrow{\mathrm{R}} l m, j}(\overrightarrow{\mathrm{k}})\right|^{2}
$$

and, according to (7c), the projected densities of states are given by

$$
\begin{aligned}
N_{t^{\prime} l^{\prime}}(E)= & \dot{P}_{t^{\prime} l^{\prime}}(E) n_{t^{\prime} l^{\prime}}^{\prime}(\overrightarrow{\mathrm{P}}(E)) \\
= & \frac{d\left(-P_{t^{\prime} l^{\prime}}^{-1}\right)}{d E} \sum_{j} V_{\mathrm{BZ}}^{-1} \\
& \times \int d^{3} k \delta\left(f_{j}(\overrightarrow{\mathrm{k}}, \overrightarrow{\mathrm{P}}(E))\right)\left|S_{t^{\prime} l^{\prime}}^{t l}(\overrightarrow{\mathrm{k}})\right|_{j}^{2}
\end{aligned}
$$

when $t^{\prime} l^{\prime} \neq t l$. Moreover,

$$
\begin{aligned}
N_{t l}(E) & =\dot{P}_{t l}(E) n_{t l}^{\prime}(\overrightarrow{\mathrm{P}}(E)) \\
& =\dot{P}_{t l}(E) \sum_{j} V_{\mathrm{BZ}}^{-1} \int d^{3} k \delta\left(f_{j}(\overrightarrow{\mathrm{k}}, \overrightarrow{\mathbf{P}}(E))\right),
\end{aligned}
$$

where, according to (8),

$$
\begin{aligned}
& \dot{P}_{t l}(E) \delta\left(f_{j}(\overrightarrow{\mathrm{k}}, \overrightarrow{\mathrm{P}}(E))\right) \\
& =\delta\left(E-E_{j}(\overrightarrow{\mathrm{k}})\right)\left(1+\sum_{t^{\prime} l^{\prime}} \frac{d\left(-P_{t^{\prime} l^{\prime}}^{-1}\right) / d E}{d P_{t l} / d E}\left|S_{t^{\prime} l^{\prime}}^{t l}(\overrightarrow{\mathrm{k}})\right|_{j}^{2}\right)^{-1}
\end{aligned}
$$

and the total density of states is given by (10).

The total shift in the $P_{t l}$ direction of the $t l$-like canonical eigenvalue surfaces caused by weak hybridization with the $t^{\prime} l^{\prime}$ partial waves is

$$
\sum_{j}^{n(t l)}\left|S_{t^{\prime} l^{\prime}}^{t l}\right|_{j}^{2} P_{t^{\prime} l^{\prime}}^{-1} \equiv\left|S_{t^{\prime} l^{\prime}}^{t l}\right|^{2} P_{t^{\prime} l^{\prime}}^{-1},
$$

where the $j$ sum is given by the simple formula

$$
\begin{aligned}
\left|S_{t^{\prime} l^{\prime}}^{t l}\right|^{2}= & \sum_{\overrightarrow{\mathrm{R}}^{\prime} m^{\prime}}^{h\left(t^{\prime} l^{\prime}\right)} \sum_{\overrightarrow{\mathrm{R}} m}^{h(t l)}\left|S_{\overrightarrow{\mathrm{R}}^{\prime} l^{\prime} m^{\prime}, \overrightarrow{\mathrm{R}} l m}\right|^{2} \\
= & \frac{4\left(2 l^{\prime}+1\right)(2 l+1)\left(2 l^{\prime}+2 l\right) !}{\left(2 l^{\prime}\right) !(2 l) !} \\
& \times \sum_{\overrightarrow{\mathrm{R}}}^{h(t)} \sum_{\overrightarrow{\mathrm{R}}^{\prime}}^{h\left(t^{\prime}\right)}\left(\frac{s}{\left|\overrightarrow{\mathrm{R}}-\overrightarrow{\mathrm{R}}^{\prime}\right|}\right)^{2\left(l^{\prime}+l+1\right)} .
\end{aligned}
$$

For $t=t^{\prime}$, the double sum over $\overrightarrow{\mathrm{R}}$ and $\overrightarrow{\mathrm{R}}^{\prime}$ excludes $\overrightarrow{\mathrm{R}}=\overrightarrow{\mathrm{R}}^{\prime}$. For a crystal, ${ }^{7} h(t)$ may be taken as the number of type- $t$ atoms per primitive cell and, in the $j$ sums on the left-hand sides of (16) and (21), we must then include the average over the Brillouin zone. While $\vec{R}$ runs over all type- $t$ atoms in the cell, $\overrightarrow{\mathrm{R}}^{\prime}$ must run over all type- $t^{\prime}$ atoms in the entire crystal.

If the hybridization is so weak that the denominators in (19a) and (19d) essentially equal unity and if, over the range of the $t l$ band, $\Delta_{t l}(E) /\left(C_{t^{\prime} l^{\prime}}\right.$ $-E)^{2}$ is essentially independent of $E$, then the entire $t l$ band contributes

$$
\Delta_{t^{\prime} l^{\prime}} \Delta_{t l}\left|S_{t^{\prime} l^{\prime}}^{t l}\right|^{2} /\left(C_{t^{\prime} l^{\prime}}-C_{t l}\right)^{2}
$$

electrons with a given spin and with angular momentum $l^{\prime}$ to the spheres of type $t^{\prime}$.

\section{PURE Mo $d$ STATES}

In Fig. 3 we show the pure Mo $d$ levels, $S_{\text {Mo } d, j}$, for an isolated, perfect $\mathrm{Mo}_{6}$ octahedron and for an isolated, real $\mathrm{Mo}_{6}$ octahedron, together with the pure Mo $d$ bands, $S_{\text {Mo } d, j}(\overrightarrow{\mathrm{k}})$, for a crystal of real octahedra. These results were obtained by diagonalizing the $30 \times 30$ submatrix of the structure constants, thereby neglecting the hybridization with all other than the Mo $d$ states, and they confirm that the Mo $d$ bands consist of narrow, weakly interacting subbands, each one being related to a level for the isolated octahedron. ${ }^{11}$

There are 20, 22, and 23 Mo electrons in $\mathrm{Mo}_{6} X_{8}$, $M \mathrm{Mo}_{6} X_{8}$, and $M \mathrm{Mo}_{6} X_{7.5}$ respectively, provided that the $M p$ bands lie entirely above, and the $X p$ and the $M s$ bands lie entirely below, the Fermi level. That is, $M$ acts as a divalent cation, and $X$ acts as a divalent anion. The oxydation state of Mo is therefore $q=\frac{16}{6}, \frac{14}{6}$, and $\frac{13}{6}$ for the abovementioned compounds. In Fig. 3 the Fermi level is placed between the bonding ${ }^{12} E_{g}$ and $T_{2 u}$ bands for $\mathrm{Mo}_{6} X_{8}$ and in the $T_{2 u}$ band for $M \mathrm{Mo}_{6} X_{8}$ and $M \mathrm{Mo}_{6} X_{7.5}$. But, as we shall see in Sec. IV, the covalent mixing with the $X p$ states will change the order and width of the Mo $d$ subbands such that the Fermi level for a wide range of potential parameters will fall in the $E_{g}$ and $A_{1 g}$ bands and, for the distorted $\mathrm{Mo}_{6} X_{8}$ compounds $(c \neq 0)$, possibly also in the $A_{u}$-band splitoff from the $T_{2 u}$ band. We now give a detailed discussion of the results 
shown in Fig. 3.

The extent of the Mo $d$ energies is most easily estimated from (16) and (22). For an isolated, perfect octahedron the extent in units of $\Delta_{\text {Mo } d}$ is $W=\left[12 \times 1400 \times 4(s \sqrt{2} / b)^{10}\right]^{1 / 2}=13.4$ because each of the six equivalent Mo atoms has four nearest neighbors at the distance $b / \sqrt{2} \equiv R_{\text {Mo-Mo }}^{\text {intra }}$, and the interaction with the next nearest neighbor at the distance $b$ can be neglected. As expected, this canonical width is approximately $\sqrt{3}$ times smaller than the canonical $d$-band width ${ }^{6}$ of 23.5 in an fcc crystal where the number of nearest neighbors is three times larger. In a bcc crystal like Mo metal with the same atomic volume, i.e., same $s$, the canonical $d$-band width is 23.8. Using the value of $\Delta_{\text {Mo } d}$ given in Table III, we estimate that

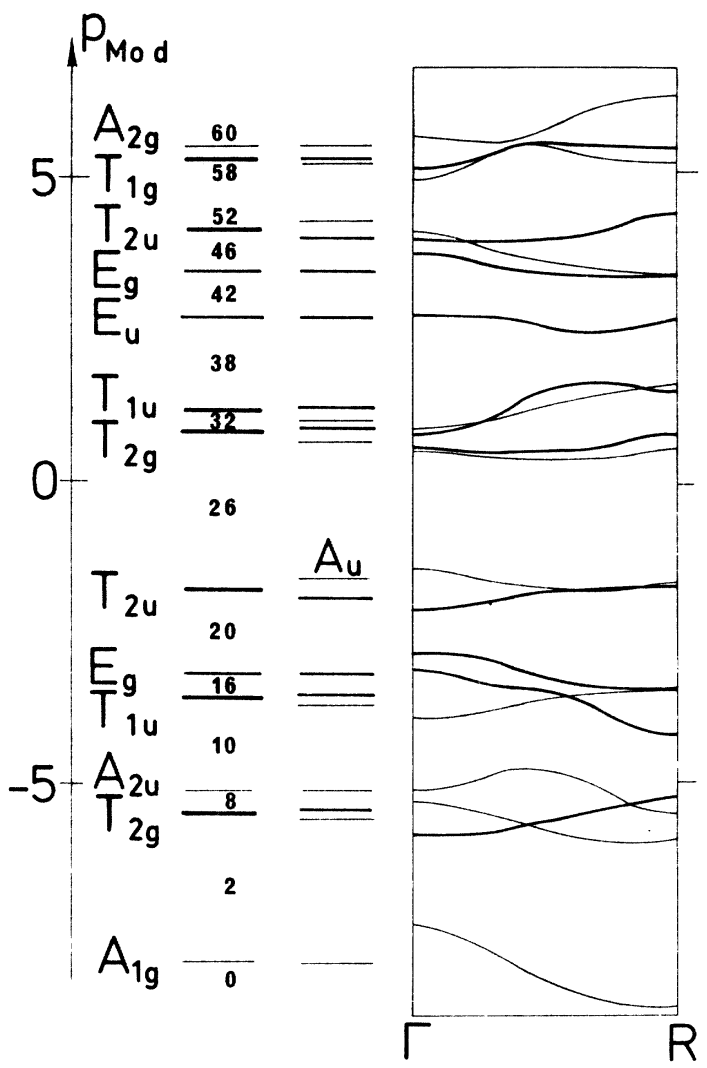

FIG. 3. Mo $d$ levels and bands obtained neglecting hybridization with all other states. On the $P_{\text {Mod }}$ scale, one unit is approximately $35 \mathrm{mRy}$. First column: levels for an isolated, perfect $(c=0)$ octahedron labeled according to the irreducible representations of $o_{h}$. In Bouckaert-Smoluchowski-Wigner notation the labeling is $\Gamma_{1}, \Gamma_{25^{\prime}}, \Gamma_{2^{\prime}}, \Gamma_{15}, \Gamma_{12}, \Gamma_{25}, \Gamma_{25^{\prime}}, \Gamma_{15}, \Gamma_{12^{\prime}}, \Gamma_{12}$, $\Gamma_{25}, \Gamma_{15^{\prime}}$, and $\Gamma_{2}$. Second column: levels for an isolated octahedron, distorted as in $\mathrm{PbMo}_{6} \mathrm{~S}_{7.5}(c / b=0.004)$. Third column: bands along the [111] direction for the octahedra in $\mathrm{PbMo}_{6} \mathrm{~S}_{7 \circ 5}(a / b=1.713, c / b=0.004, \phi$ $=25.8^{\circ}$; see text in caption of Fig. 1). The numbers between the levels in the first column indicate the electron count. the extent of the $d$ levels for an isolated $\mathrm{Mo}_{6}$ octahedron is $0.5 \mathrm{Ry}$.

Considering now a crystai of $\mathrm{Mo}_{6}$ octahedra, there will be an extra contribution to (22) from the intercluster terms and, retaining only the shortest intercluster bonds, of which there is one per Mo atom, we obtain $\partial W^{2} / W^{2}=\frac{1}{4}\left(R_{\mathrm{Mo}-\mathrm{Mo}}^{\text {intra }} / R_{\mathrm{Mo}-\mathrm{Mo}_{0}}^{\text {inter }}\right)^{10}$. This extra contribution goes into band broadening of the levels for the isolated octahedron and to repulsion between these subbands such that $\partial W^{2} / W^{2}=(w / W)^{2}$ $+2 \partial E /(E-C)$. Here, $w$ is an average subband width and $\partial E /(E-C)$ is an average repulsion constant. An upper bound on the subband width is therefore given by

$$
w \leqslant \frac{1}{2}\left(R_{\mathrm{Mo}-\mathrm{Mo}}^{\mathrm{intra}} / R_{\mathrm{Mo}-\mathrm{Mo}}^{\text {inter }}\right)^{5} W,
$$

which, for the compounds listed in Table I in order of increasing $a / b$, namely, $\mathrm{Mo}_{6} \mathrm{~S}_{8}, \mathrm{Mo}_{6} \mathrm{Se}_{8}$, $\mathrm{PbMo}_{6} \mathrm{~S}_{7.5}, \mathrm{PbMo}_{6} \mathrm{Se}_{8}$, and $\mathrm{Mo}_{6} \mathrm{Te}_{8}$, takes the respective values $4.1,2.9,2.5,1.9$, and 1.6. For $\mathrm{PbMO}_{6} \mathrm{~S}_{7.5}$ the full calculation shown in Fig. 3 yields $w \approx 0.7 \ll 2.5$. The intercluster contribution to $(22)$ must therefore be dominated by the subband repulsion $\partial E /(E-C)$, although its value is less than $2 \%$ and the corresponding shifts can barely be detected in the figure. Having discussed the gross features of the Mo $d$ states, we now consider certain states of particular interest.

\section{A. $d$ states for the isolated octahedron}

For the isolated, perfect octahedron the $d$ state of lowest energy belongs to the irreducible representation $A_{1 g}$ of $O_{h}$. It has the symmetry

$$
\begin{aligned}
\left(3 z^{2}-r^{2}\right)_{z} & +\left(3 z^{2}-r^{2}\right)_{-z}+\left(3 x^{2}-r^{2}\right)_{x} \\
& +\left(3 x^{2}-r^{2}\right)_{-x}+\left(3 y^{2}-r^{2}\right)_{y}+\left(3 y^{2}-r^{2}\right)_{-y},
\end{aligned}
$$

where a subscript indicates the position of the orbital. Retaining the interactions with the nearest neighbors only and using Table II, its energy is

$$
\begin{aligned}
4 S\left(3 z^{2}-r^{2}, 3 x^{2}-r^{2} ; \frac{1}{2} b, 0, \frac{1}{2} b\right) & =-570 \sqrt{2}(s / b)^{5} \\
& =-7.3
\end{aligned}
$$

in units of $\Delta_{\text {Mo } d}$ and relative to $C_{\text {Mo } d}$, i.e., on $P_{\text {Mo } d}$ scale.

The $A_{2 u}$ state is

$$
x y_{z}-x y_{-z}+y z_{x}-y z_{-x}+z x_{y}-z x_{-y}
$$

and has the energy

$4 S\left(-x y, y z ; \frac{1}{2} b, 0, \frac{1}{2} b\right)=-400 \sqrt{2}(s / b)^{5}=-5.2$.

Two sets of doubly degenerate $E_{g}$ states are

$$
\begin{aligned}
\left(x^{2}-y^{2}\right)_{z}+\left(x^{2}-y^{2}\right)_{-z} & \\
& +\exp \left(\mp \frac{2}{3} \pi i\right)\left[\left(y^{2}-z^{2}\right)_{x}+\left(y^{2}-z^{2}\right)_{-x}\right] \\
& +\exp \left( \pm \frac{2}{3} \pi i\right)\left[\left(z^{2}-x^{2}\right)_{y}+\left(z^{2}-x^{2}\right)_{-y}\right]
\end{aligned}
$$


and

$$
\begin{aligned}
\left(3 z^{2}-r^{2}\right)_{z} & +\left(3 z^{2}-r^{2}\right)_{-z} \\
& +\exp \left(\mp \frac{2}{3} \pi i\right)\left[\left(3 x^{2}-r^{2}\right)_{x}+\left(3 x^{2}-r^{2}\right)_{-x}\right] \\
& +\exp \left( \pm \frac{2}{3} \pi i\right)\left[\left(3 y^{2}-r^{2}\right)_{y}+\left(3 y^{2}-r^{2}\right)_{-y}\right] .
\end{aligned}
$$

Neglecting the hybridization between them, their energies are, respectively,

$$
\begin{aligned}
-2 S\left(x^{2}-y^{2}, y^{2}-z^{2} ; \frac{1}{2} b, 0, \frac{1}{2} b\right) & =-215 \sqrt{2}(s / b)^{5} \\
& =-2.8
\end{aligned}
$$

and

$$
\begin{aligned}
-2 S\left(3 z^{2}-r^{2}, 3 x^{2}-r^{2} ; \frac{1}{2} b, 0, \frac{1}{2} b\right) & =285 \sqrt{2}(s / b)^{5} \\
& =3.7 .
\end{aligned}
$$

Including the hybridization, we find that the two states mix in the ratio $21 i / 100$ such that the energy of the bonding $E_{g}$ state, having primarily $x^{2}-y^{2}$ character, is

$$
-(215+441 / 20) \sqrt{2}(s / b)^{5}=-3.1,
$$

while the energy of the antibonding $E_{g}$ state, with dominating $3 z^{2}-r^{2}$ character, is

$(285+441 / 20) \sqrt{2}(s / b)^{5}=4.0$.

One of the $E_{g}\left(x^{2}-y^{2}\right)$ states is shown schematically in Fig. 4.

Two sets of triply degenerate $T_{2 u}$ states are

$$
\begin{aligned}
& \left\{\left(x^{2}-y^{2}\right)_{z}-\left(x^{2}-y^{2}\right)_{-z},\left(y^{2}-z^{2}\right)_{x}-\left(y^{2}-z^{2}\right)_{-x},\right. \\
& \left.\left(z^{2}-x^{2}\right)_{y}-\left(z^{2}-x^{2}\right)_{-y}\right\}
\end{aligned}
$$

and

$$
\begin{aligned}
& \left\{z y_{y}-z y_{-y}-z x_{x}+z x_{-x}, x z_{z}-x z_{-z}-x y_{y}+x y_{-y},\right. \\
& \left.y x_{x}-y x_{-x}-y z_{z}+y z_{-z}\right\}
\end{aligned}
$$

with the respective energies 0 and

$$
2 S\left(-x z,-x y ; 0, \frac{1}{2} b, \frac{1}{2} b\right)=200 \sqrt{2}(s / b)^{5}=2.6 \text {. }
$$

These states, however, $\operatorname{mix}$ in the ratio $\frac{1}{3}(\sqrt{11}-\sqrt{2})$ $=0.63$, whereby the energy of the bonding $T_{2 u}$ state, having primarily $x^{2}-y^{2}$ character, becomes $-100(\sqrt{11}-\sqrt{2})(s / b)^{5}=-1.7$, while that of the antibonding state, with dominating $(x z, y z)$ character, becomes $100(\sqrt{11}+\sqrt{2})(s / b)^{5}=4.3$. By transferring the bonding $T_{2 u}$ level, which is occupied, from the upper to the lower edge of the gap in the middle of the Mo $d$ spectrum, this mixing is partly responsible for the stability of the octahedron. One of the bonding $T_{2 u}$ states is shown in Fig. 5 .

The $d$ state of highest energy is the $A_{2 g}$ state with symmetry

$$
\left(x^{2}-y^{2}\right)_{z}+\left(x^{2}-y^{2}\right)_{-z}+\left(y^{2}-z^{2}\right)_{x}
$$$$
\text { and energy }
$$
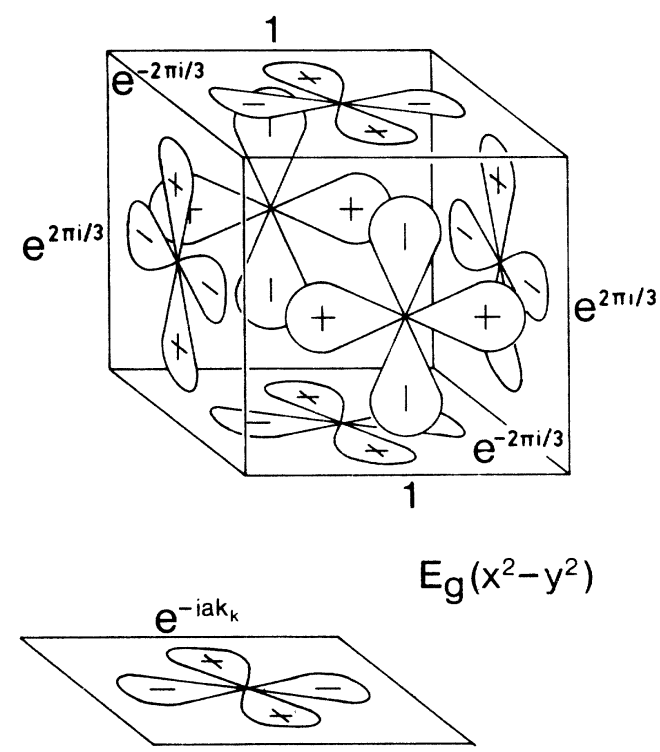

FIG. 4. Symmetry of one of the $E_{g}\left(x^{2}-y^{2}\right)$ states.

$4 S\left(x^{2}-y^{2}, y^{2}-z^{2} ; \frac{1}{2} b, 0, \frac{1}{2} b\right)=430 \sqrt{2}(s / b)^{5}=5.5$.

The difference between the highest and lowest $d$ energies for an isolated, perfect octahedron is therefore $1000 \sqrt{2}(s / b)^{5}=12.9$, and this is in accord with the estimate $W=13.4$ given above.

In the molybdenum chalcogenides, the binaries

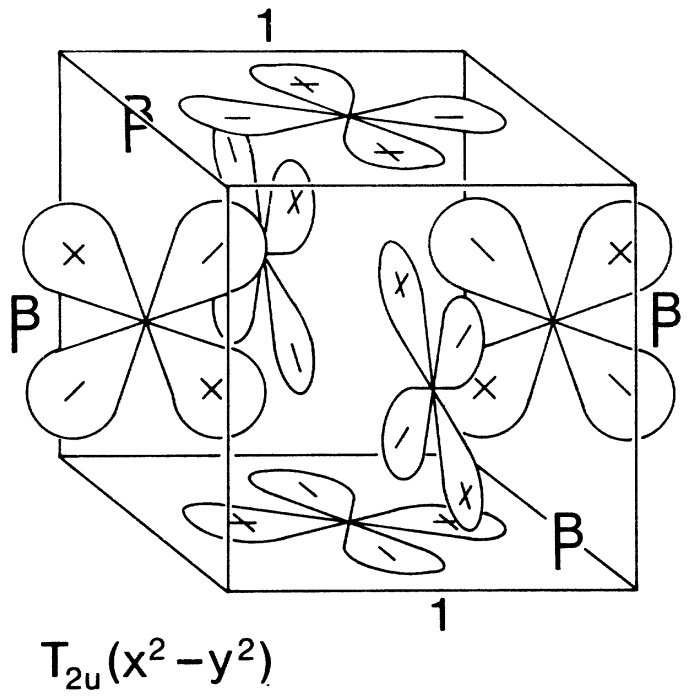

FIG. 5. Symmetry of one of the $T_{2 u}$ states. The coefficient of an $x z$ or $y z$ orbital is $\beta / \sqrt{2}$ times that of an $x^{2}-y^{2}$ orbital, with $\beta=\frac{1}{3}(\sqrt{11}-\sqrt{2})=0.63$. That $T_{2 u}$ state which transforms according to $A_{u}$ of $C_{3 i}$ is the state shown plus the two obtained from it by cyclically permuting $x, y$, and $z$. At the $\left(0,0, \frac{1}{2} b\right)$ site and for the $A_{u}$ state, the coefficient of the $(x-y) z / \sqrt{2}$ orbital is $\beta$ times that of the $x^{2}-y^{2}$ orbital. 
in particular, the octahedra are slightly elongated along the [111] axis such that the Mo atoms are displaced from the face centers by the amount $c / \sqrt{2}$ along the face diagonals (see Fig. 1 and Table I). The elongation lowers the symmetry of an isolated octahedron from $O_{h}$ to $C_{3 i}$ and thereby splits all threefold, but no other, degeneracies, i.e., $T_{u} \rightarrow E_{u}+A_{u}$ and $T_{g} \rightarrow E_{g}+A_{g}$. For the $T_{2 u}$ levels the sum of the three partner functions, defined as in Fig. 5, will belong to the irreducible representation $A_{u}$ of $C_{3 i}$ and the remaining two functions will belong to $E_{u}$. Whereas the interactions of the $\left(x^{2}-y^{2}\right)_{z}$ orbital with the $\left(y^{2}-z^{2}\right)_{x}$, $-\left(y^{2}-z^{2}\right)_{-x},\left(z^{2}-x^{2}\right)_{y}$, and $-\left(z^{2}-x^{2}\right)_{-y}$ orbitals cancel for a perfect octahedron, this is not true for an elongated octahedron, where we find that the energy of the bonding $A_{u}$ level is raised by ${ }^{13}$ $3460 c / b(s / b)^{5}$ such that the splitting between the bonding $A_{u}$ and $E_{u}$ levels is $5190 c / b(s / b)^{5}$. With the values of $c / b$ given in Table I this splitting is 0 and 0.2 in $\mathrm{PbMo}_{6} \mathrm{Se}_{8}$ and $\mathrm{PbMo}_{6} \mathrm{~S}_{7.5}$, respectively, but it is about 2 in the binaries, and this will exceed the crystalline bandwidth.

\section{B. Band states}

The Mo $d$ bands for $\mathrm{PbMo}_{6} \mathrm{~S}_{7.5}$ shown in the righthand panel of Fig. 3 and in the bottom panel of Fig. 6 were obtained by diagonalizing numerically the $30 \times 30$ submatrix $^{7} S(\vec{k})$ at points in the simple cubic Brillouin zone. Some details of these bands are due to hybridization between Bloch sums of nondegenerate $d$ states for the isolated octahedron, and those features are sensitive to the relative positions of the octahedron $d$ levels and would consequently change if the covalent mixing with the $X p$ states were included. There is, however, for each band a characteristic shape and width which, we believe, would survive in a complete calculation, and those characteristics derive from the nature of the $d$ states for the isolated octahedron. As an example we show in Fig. 6, from the bottom and up, the Mo $d$ bands in the vicinity of the bonding $E_{g}$ and $T_{1 u}$ levels, the band derived from the Bloch sums of the two bonding $E_{g}$ states only, and the band derived from the Bloch functions shown in Fig. 4 of the two $E_{g}\left(x^{2}-y^{2}\right)$ states only, i.e.,

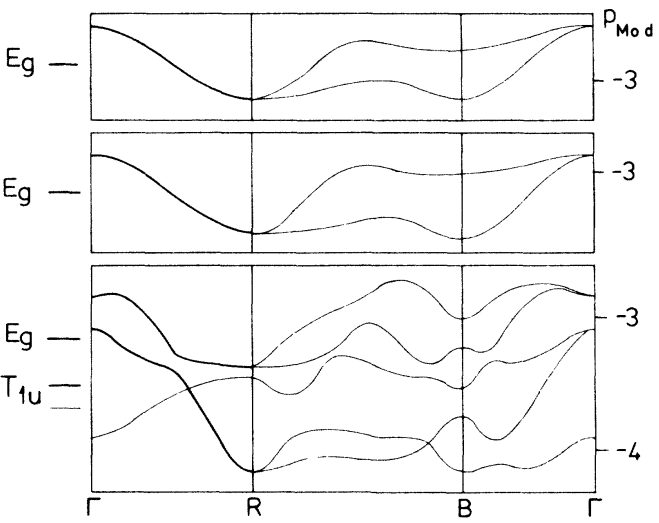

FIG. 6. Pure $d$ subbands of $\mathrm{PbMo}_{6} \mathrm{~S}_{7.5}$ octahedra. From the bottom and up, these bands were obtained by (1) retaining the interactions between all 30 states of the octehedron and between all octahedra in the crystal, (2) retaining the interaction between the two degenerate states of the bonding $E_{g}$ level only and retaining the interactions between all octahedra in the crystal, (3) retaining the interaction between the two $E_{8}$ $\left(x^{2}-y^{2}\right)$ states only and retaining the nearest-neighbor inter- and intracluster interactions only, i.e., using (26a). The simple cubic Brillouin zone is shown in Fig. 12 .

neglecting the mixing with the $3 z^{2}-r^{2}$ states. The latter band is of particular relevance because the $3 z^{2}-r^{2}$ admixture will be strongly suppressed by the $X p$ covalent mixing.

This doubly degenerate $E_{g}\left(x^{2}-y^{2}\right)$ band, which we believe is the essential conduction band in the Chevrel-phase molybdenum chalcogenides, has the analytical form

$$
P_{\text {Mo } d}(E)=\left(E-C_{\text {Mo } d}\right) / \Delta_{\text {Mo } d}=S^{\text {intra }}+S^{\text {inter }} G(\overrightarrow{\mathrm{k}}),
$$

where $S^{\text {intra }}=-215 \sqrt{2}(s / b)^{5}$, as previously mentioned, and we have retained the smallest intraand intercluster Mo-Mo bonds only. The intercluster coupling constant $S^{\text {inter }}=S\left(x^{2}-y^{2}, x^{2}-y^{2}\right.$; $\overrightarrow{\mathrm{R}}^{\text {inter }}$ ) may be found in Table II as a function of the Mo-Mo intercluster vector expressed in the $x y z$ coordinate system (see Fig. 1), i.e.,

$$
R^{\text {inter }}(l, m, n)=\left(\frac{1}{3}(1-\cos \phi-\sqrt{3} \sin \phi) a-\mathrm{c}, \frac{1}{3}(1-\cos \phi+\sqrt{3} \sin \phi) a-c, \frac{1}{3}(1+2 \cos \phi) a-b\right)
$$

in terms of the lattice constant $a$ the dimensions $b$ and $c$ of the octahedron, and the turn angle $\phi$. The dispersion of the band is independent of $b, c$, and $\phi$ and is given by

$$
G(\overrightarrow{\mathrm{k}})=\frac{1}{3}\left\{i+j+k \pm[i(i-j)+j(j-k)+k(k-i)]^{1 / 2}\right\},
$$

and the two eigenvectors combining the two $E_{g}$ 
states of the octahedron (Fig. 4) to the Bloch states for the two $E_{g}$ bands are

$$
\tilde{U}(\overrightarrow{\mathrm{k}})=(1 / \sqrt{2})\{\exp (i u),+1\},(1 / \sqrt{2})\{\exp (i u),-1\},
$$

where

$\cos u(\overrightarrow{\mathrm{k}})=\left[k-\frac{1}{2}(i+j)\right][i(i-j)+j(j-k)+k(k-i)]^{-1 / 2}$.

In (26), $i \equiv \cos \left(a k_{i}\right)$, etc., and $k_{i}, k_{j}$, and $k_{k}$ are the components of $\overrightarrow{\mathrm{k}}$ in the $i j k$ coordinate system. Although the point group is $C_{3 i}$, the $E_{g}\left(x^{2}-y^{2}\right)$ band has full cubic symmetry. In (26a) the function $i+j+k$ of $\overrightarrow{\mathrm{k}}$ changes sign under the translation $(1,1,1) \pi / a$ such that $G(\vec{k})$ has a density of states symmetric about zero (Fig. 7). Moreover, $G(\overrightarrow{\mathrm{k}})$ extends from -1 to +1 and the $E_{g}\left(x^{2}-y^{2}\right) \mid$ bandwidth is therefore $2\left|S^{\text {inter }}\right|$, where $S^{\text {inter }}=0.53,0.31,0.28$, 0.18 , and 0.14 for $\mathrm{Mo}_{6} \mathrm{~S}_{8}, \mathrm{Mo}_{6} \mathrm{Se}_{8}, \mathrm{PbMo}_{8} \mathrm{~S}_{7.5}$, $\mathrm{PbMo}_{6} \mathrm{Se}_{8}$, and $\mathrm{Mo}_{6} \mathrm{Te}_{8}$, respectively. This trend clearly follows the decrease of $\left(R_{\mathrm{Mo}-\mathrm{MO}_{0}}^{\ln \mathrm{ra}} / R_{\mathrm{Mo}-\mathrm{M}_{0}}^{\text {inter }}\right)^{5}$ associated with the increase of $a / b$. The bandwidths in rydbergs may be found in Table $\mathrm{V}$.

The $E_{g}\left(x^{2}-y^{2}\right)$ band depends in a rather interesting way on the rotation $\phi$ of the octahedron with respect to the lattice (see Figs. 1 and 4). For $\phi$ $=0$, the intercluster distance is $a-b$, which is as small as the intracluster distance, and $S^{\text {inter }}$ $=-10(a / b-1)^{-5}(s / b)^{5}=-0.88,-0.53,-0.49,-0.33$, and -0.25 for the five above-mentioned compounds. The band is therefore rather broad and $\vec{k}=0$ has the lowest energy. As $\phi$ is increased, the $x^{2}-y^{2}$ orbitals are shifted away from each other, with the result that the interaction changes sign at about $\phi=15^{\circ}$, whereby the band goes through a collapse

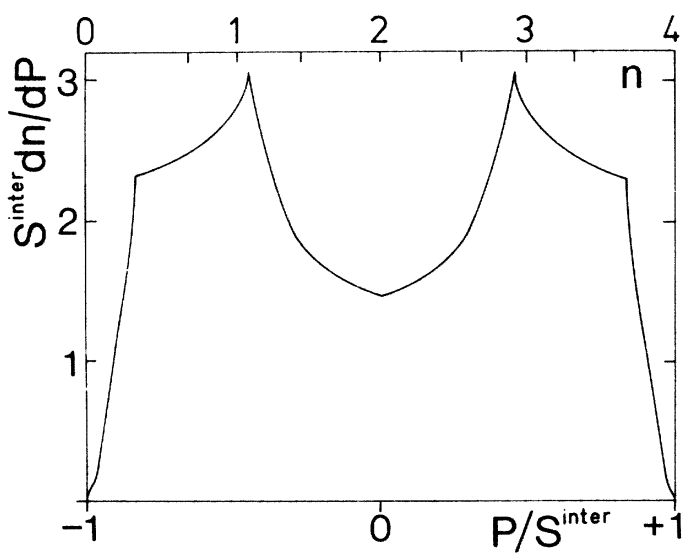

FIG. 7. Density of states in the $E_{g}\left(x^{2}-y^{2}\right)$ subband (26). Bandwidths estimated for various compounds are given in Table $\mathrm{V}$. The number of $E_{g}$ electrons per cell is $n$. We estimate that $n \approx 3.1$ and 2.1 for $\mathrm{PbMo}_{6} \mathrm{~S}_{7.5}$ and $\mathrm{PbMo}_{6} \mathrm{Se}_{8}$, respectively; the corresponding densities of states at the Fermi level may be found in Table V. and inverts. The bandwidth hereafter rises to a maximum near the observed value of $\phi$ and it finally decreases with the strongly increasing intercluster distance. This is shown in Fig. 8.

In Sec. IV we shall demonstrate that the covalent mixing with the $X p$ states will lift the $E_{g}$ band above the $T_{2 u}$ band and the possibility therefore exists that, in the binaries, the $E_{g}$ band will be close to, or overlap, the $A_{u}$ band split off from the $T_{2 u}$ band. We shall therefore estimate the width of this $A_{u}$ band. Neglecting the hybridization with any other $d$ state for the isolated octahedron, the $A_{u}$ band may be expressed as (24), where $S^{\text {intra }}$ is now the $A_{u}$ level and where $G(\overrightarrow{\mathrm{k}})=\frac{1}{3}(i+j+k)$ such that the bandwidth is $2\left|S^{\text {inter }}\right|$. The intercluster coupling constant is

$$
\begin{aligned}
S^{\text {inter }}=\left(1+\beta^{2}\right)^{-1} & {\left[S\left(y^{2}-x^{2}, x^{2}-y^{2}\right)\right.} \\
& +2 \beta S\left(y^{2}-x^{2},(x-y) z / \sqrt{2}\right) \\
& \left.+\beta^{2} S((y-x) z / \sqrt{2},(x-y) z / \sqrt{2})\right],
\end{aligned}
$$

which may be evaluated using (25) and Table II. The admixture $\beta$ of the $(x-y) z$ orbitals turns out to be crucial: For $\beta=0$, the intercluster coupling constant would just be the negative of that for the $E_{g}$ band, and the width of the singly degenerate $A_{u}$ band would consequently equal that of the doubly degenerate $E_{g}$ band, which is $2 \times 0.31$ for $\mathrm{Mo}_{6} \mathrm{Se}_{8}$. The intercluster $(x-y) z$ interaction is, however, several times larger than the $x^{2}-y^{2}$ interaction and of opposite sign. In $\mathrm{Mo}_{6} \mathrm{Se}_{8}$ the intercluster coupling constant in fact vanishes for $\beta=0.53$ and, with the previously found value of $\beta=0.63$, we obtain $S^{\text {inter }}=0.1$. This very narrow band will be broadened by covalent mixing with the $X p$ states.

Also the $A_{1 g}$ band may be expressed by (24) with $G(\overrightarrow{\mathrm{k}})=\frac{1}{3}(i+j+k)$, and we find that $S^{\text {inter }}=0.77$, $0.53,0.49,0.36$, and 0.30 for the respective compounds listed in Table $\mathrm{V}$. In the following section we shall see that this band is considerably broadened by covalent mixing.

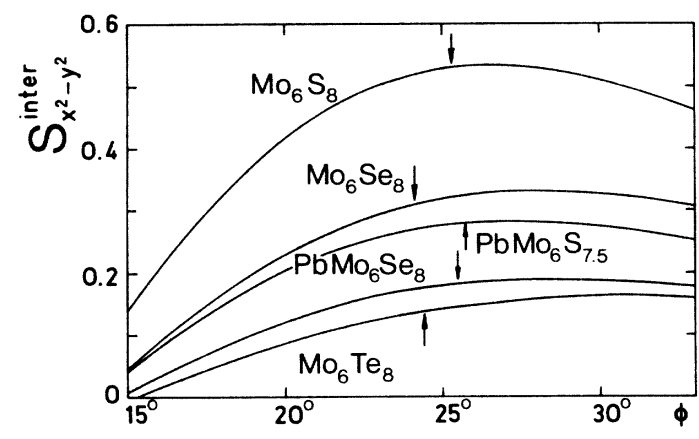

FIG. 8. Dependence of the $x^{2}-y^{2}$ direct intercluster coupling constant on the turn angle $\phi$ defined in Fig. 1 . The observed turn angles are indicated by arrows. 


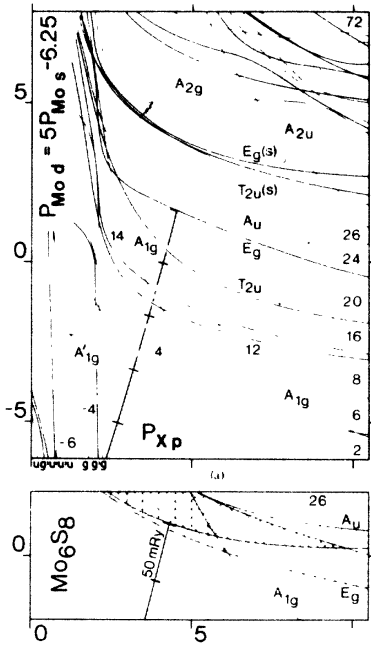

(a)

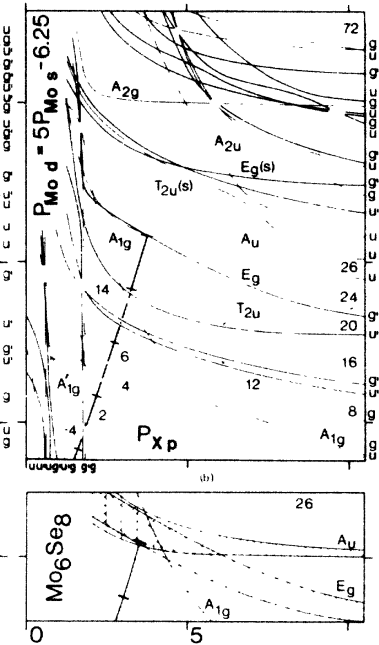

(b)

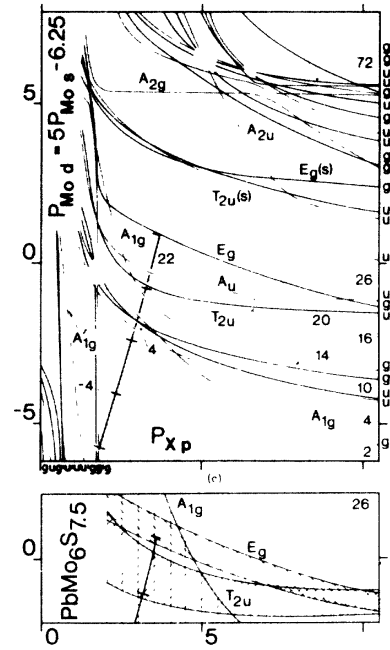

(c)

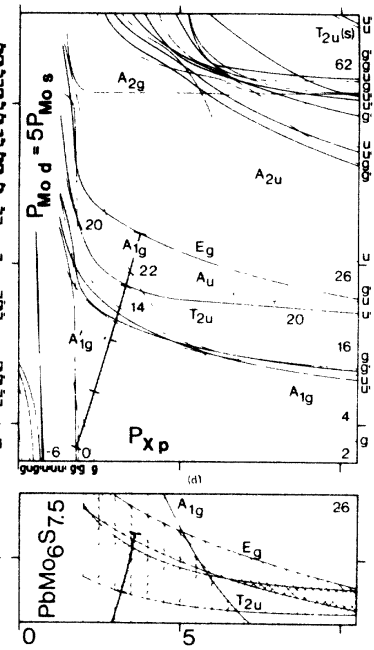

(d)

FIG. 9. Cross sections of the canonical eigenvalue surfaces in $\left\{P_{M o s}, P_{M o d}, P_{X p}\right\}$ space for the $\mathrm{Mo}_{6} X_{14}$ clusters appropriate for (a) $\mathrm{Mo}_{6} \mathrm{~S}_{8}$, (b) $\mathrm{Mo}_{6} \mathrm{Se}_{8}$, and (c), (d) $\mathrm{PbMo}_{6} \mathrm{~S}_{7.5}$. The planes of cross section are parallel to the $P_{X p}$ axis and have been chosen in such a way that they lie close to the self-consistently estimated potential paths (e.g., the full path in Fig. 10). The equations of these planes are $P_{\text {Mod }}-5 P_{\text {Mos }}=-6.25$ for (a) - (c) and $P_{\text {Mod }}-5 P_{\text {Mos }}=0$ for (d). The eigenvalue surfaces are labeled according to their parity and degeneracy at the right-hand side and bottom of each figure. Hence, $g$ and $u$ denote (singly degenerate) $A_{g}$ and $A_{u}$ states, while $g^{\prime}$ and $u^{\prime}$ denote (doubly degenerate) $E_{g}$ and $E_{u}$ states. The numbers between the surfaces indicate the Mo-electron count (i.e., the electron count normalized to zero at $P_{\text {Mos }} \rightarrow-\infty, P_{M o d} \rightarrow-\infty$, and $\left.P_{X p} \rightarrow+\infty\right)$. Neglecting the trigonal distortion and labeling the levels according to the irreducible representations of the full cubic group, the levels, from the bottom and up at the right-hand side of (d), are $A_{1 g}\left(\Gamma_{1}\right), T_{1 u}\left(\Gamma_{15}\right), T_{2 g}\left(\Gamma_{25^{\circ}}\right), T_{2 u}\left(\Gamma_{25}\right), E_{g}\left(\Gamma_{12}\right), A_{2 u}\left(\Gamma_{2^{\prime}}\right), T_{2 g}\left(\Gamma_{25^{\prime}}\right), T_{1 u}\left(\Gamma_{15}\right), E_{g}\left(\Gamma_{12}\right), T_{2 u}\left(E_{u}\right)\left(\Gamma_{25}\right), E_{u}\left(\Gamma_{12^{\prime}}\right)$, $A_{2 g}\left(\Gamma_{2}\right), T_{2 u}\left(A_{u}\right)\left(\Gamma_{25}\right), T_{1 g}\left(\Gamma_{15^{\circ}}\right)$, and $T_{2 u}\left(\Gamma_{25}\right)$. The heavy curve in each figure is the projection onto the plane of cross section of the potential path defined in (2) and given by (11) with the parameters in Table III. The energy scale is marked for each $50 \mathrm{mRy}$ terminating at the Fermi level, and the potential functions for $\mathrm{PbMo}_{6} \mathrm{~S}_{7.5}$ are shown in Fig. 2. In the state defined by the intersection of the potential path, $\overrightarrow{\mathrm{P}}(E)$, with a canonical eigenvalue surface, $f_{j}(\overrightarrow{\mathrm{P}})=0$, the (un-normalized) partial probabilities are $\left(\partial f_{j} / \partial P_{t l}\right)\left(d P_{t l} / d E\right)$ (see Sec. II A). The inset at the bottom of each figure shows the band broadening of the $A_{1 g}, E_{g}$, and $A_{u}$ (or $T_{2 u}$ ) levels as estimated in Secs. III B and IV C and given in Table V.

\section{COVALENT MIXING AND HYBRIDIZATION}

In order to study the effect on the Mo $d$ levels of covalent mixing with the $X p$ waves and hybridization with the Mo $s$ waves we computed the canonical eigenvalue surfaces in $\left\{P_{\text {Mo } s}, P_{\text {Mo } d}, P_{X p}\right\}$ space for three $\mathrm{Mo}_{6} X_{14}$ clusters using the atomic positions ${ }^{3}$ in, respectively, $\mathrm{Mo}_{6} \mathrm{~S}_{8}, \mathrm{Mo}_{6} \mathrm{Se}_{8}$, and $\mathrm{PbMo}_{6} \mathrm{~S}_{7.5}$. The $\mathrm{Mo}_{6} X_{14}$ cluster is the $\mathrm{Mo}_{6}$ octahedron surrounded by its $8+6$ nearest neighbors and the presence of the divalent cation $M$ only influences our eigenvalues to the extent that it modifies the positions of the Mo and $X$ atoms and contributes two electrons to the Fermi sea.

The eigenvalue surfaces shown in Figs. 9 and 10 were traced along straight lines in $\overrightarrow{\mathbf{P}}$ space by diagonalizing the appropriate $78 \times 78$ Hamiltonian matrix (14) for fixed values of $\Delta_{M o s}, \Delta_{M o d}$, and $\Delta_{X p}$, and varying the values of $\hat{C}_{M o d}-\hat{C}_{X p}$ and $\hat{C}_{\text {Mo } d}-\hat{C}_{\text {Mo } s}$. Once constructed, these canonical eigenvalue surfaces apply to any potential path, i.e., to any set of potential parameters, as explained in Sec. II A.

For each compound the potential path shown in the figure was estimated self-consistently with the Mo- $X$ electron transfer, keeping the $\mathrm{Mo}_{6} X_{8}$ unit charged by -2 or 0 , as it would be in a solid containing one or no divalent cation per primitive cell. In all cases, each Mo sphere turned out to be neutral within a few tenths of an electron. The potential parameters are listed in Table III.

We find that the Fermi level falls in the doubly degenerate $E_{g}$ band just below the Mo $d$ gap and, for the binaries, also in the $A_{u}$ band.

We shall now discuss the covalent mixing and the hybridization in some detail and, after having considered the effects on the ordering of the Mo $d$ subbands (Sec. IV A) and on the relative band position $C_{\text {Mo } d}-C_{X p}$ via the requirement of selfconsistency (Sec. IV B), we shall estimate the effects on the widths of the Mo $d$ subbands (Sec. IV C). We conclude that, whereas the $E_{g}$ band is broadened a few times, the $A_{u}$ and the $A_{1 g}$ band- 
widths are almost entirely due to intercluster coupling via the $X$ atoms. The $A_{1 g}$ band is, in fact, so wide that it overlaps the $E_{g}$ band. These results are summarized in the bottom panels of Fig. 9, in Fig. 11, and in Table V. Finally, in Sec. IVD, we shall compare our results with those of other investigators.

\section{A. $\mathrm{Mo}_{6} \boldsymbol{X}_{14}$-cluster states}

Neglecting for the moment the Mo $s$ waves, the levels in Fig. 9 will tend towards the pure Mo $d$ levels in Fig. 3 when $P_{X_{p}} \rightarrow \pm \infty$, and they will tend towards the pure $X p$ levels when $P_{\text {Mo } d} \rightarrow \pm \infty$. The Mo $d$ asymptotes are thus centered at $P_{\text {Mo } d}=0$ and

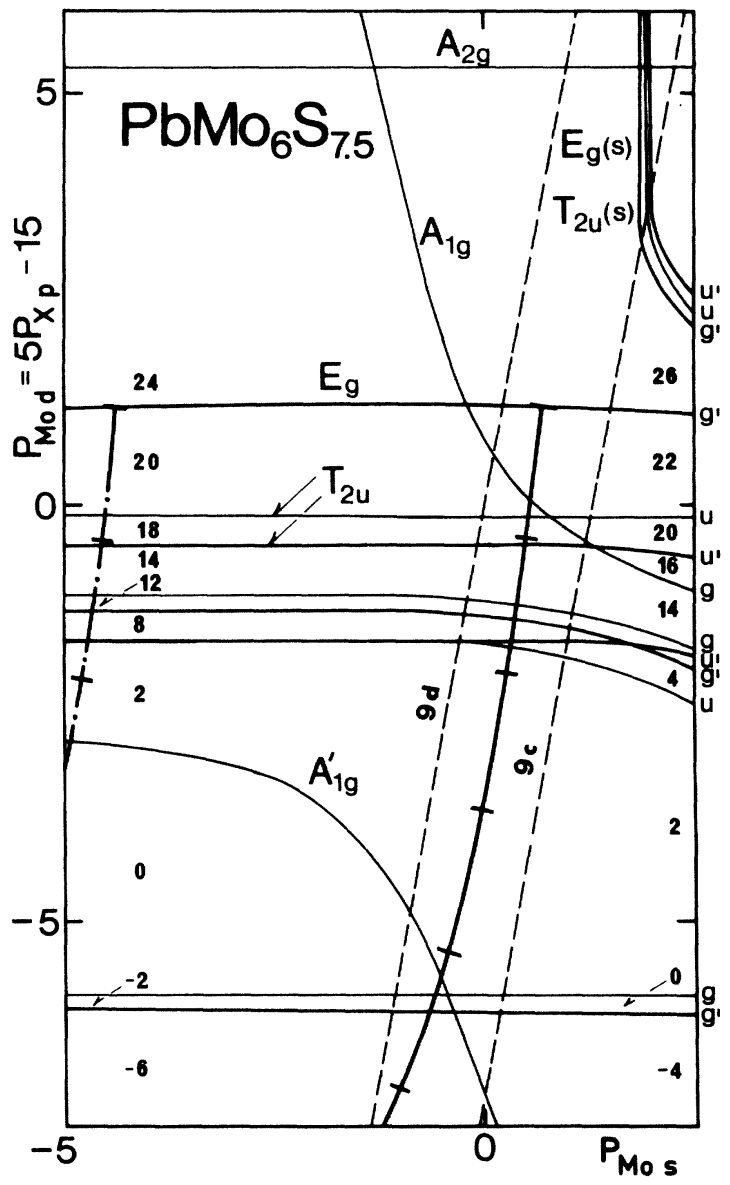

FIG. 10. Same as in Figs. 9(c) and 9(d) except that the plane of cross section is here parallel with the $P_{M o s}$ axis and has the equation $P_{M o d}-5 P_{X P}=-15$. The stippled lines indicate the traces with the planes of Figs. 9(c) and 9(d). The dot-dashed potential path includes the effect of weak hybridization with the $X s$ waves as explained in Sec. IV A. We estimate that the uncertainty in the positions of the Mo $s$-like levels, caused by the finite size of our cluster model, corresponds to an uncertainty in the position of the potential path bound by the solid and dot-dashed paths. have the approximate width $W_{\text {Mo } d}=13.4$. The $X p$ asymptotes are centered at $P_{X p}=0$ and their width, estimated from (16) and (22), is $W_{X p} \approx[12 \times 72$ $\left.\times 4\left(s / b^{\prime}\right)^{6}\right]^{1 / 2}=3.5\left(b / b^{\prime}\right)^{3}$, because each of the $14 X$ atoms has four $X$ neighbors at approximately the same distance $b^{\prime}$. Of the compounds listed in Table I, $\mathrm{Mo}_{6} \mathrm{~S}_{8}$ therefore has the largest $X p$ bandwidth, namely, 5.3. In the real crystals each $X$ atom has more than four nearest $X$ neighbors and the $X p$ bandwidths are then somewhat larger than in the $\mathrm{Mo}_{6} X_{14}$ cluster.

When $\left|P_{X p}\right| \gg W_{X p}$ the Mo $d$-like levels behave

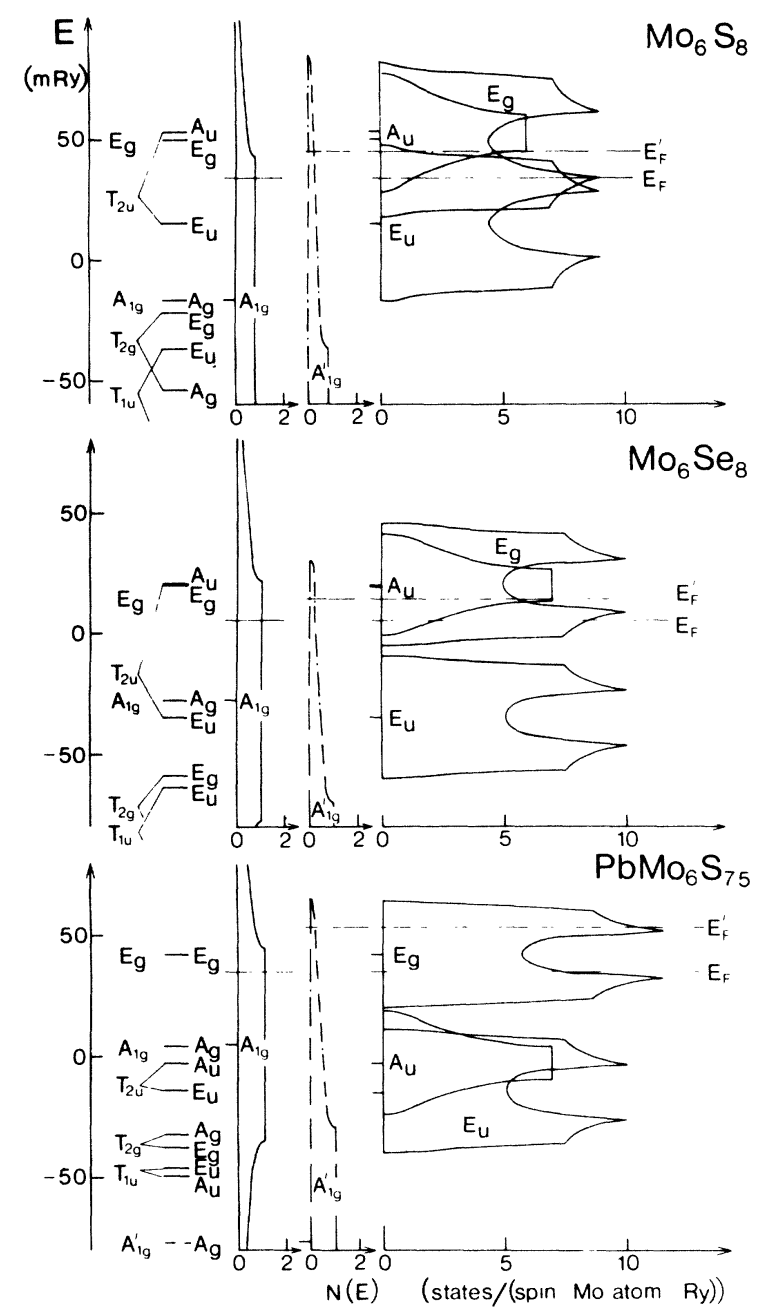

FIG. 11. Energy levels in the vicinity of the Fermi level and superposed densities of states for the $E_{g}, A_{u}$, $E_{u}$, and $A_{1 \mathrm{~g}}$ subbands in $\mathrm{Mo}_{6} \mathrm{~S}_{8}, \mathrm{Mo}_{6} \mathrm{Se}_{8}$, and $\mathrm{PbMO}_{6} \mathrm{~S}_{7.5}$. The $A_{1}$ density-of-states curves and the Fermi levels drawn in solid line correspond to the potential paths drawn in solid line in Figs. 9 and 10. The dot-dashed $A_{1 \mathrm{~g}}^{\prime}$ density-of-states curves and Fermi levels $\left(E_{F}^{\prime}\right)$ correspond to the dot-dashed potential path in Fig. 10; they include the effect of weak hybridization with the $X s$ waves. The zero of energy is at $C_{\text {Mod }}$. 
TABLE IV. Average hybridization in the Mo $d$ band.

\begin{tabular}{|c|c|c|c|c|c|}
\hline & $\mathrm{Mo}_{6} \mathrm{~S}_{8}$ & $\mathrm{Mo}_{6} \mathrm{Se}_{8}$ & $\mathrm{PbMo}_{6} \mathrm{~S}_{7.5}$ & $\mathrm{PbMo}_{6} \mathrm{Se}_{8}$ & $\mathrm{Mo}_{6} \mathrm{Te}_{8}$ \\
\hline$\frac{1}{30}\left|S_{\mathbf{M o} p}^{\mathrm{Mo} d}\right|^{2}$ & 6.3 & 6.3 & 6.3 & 6.3 & 6.3 \\
\hline$\frac{1}{30}\left|S_{X s}^{\mathrm{Mod} d}\right|^{2}$ & 1.3 & 0.9 & 1.1 & 0.8 & 0.6 \\
\hline$\frac{1}{30}\left|s_{X p}^{\mathrm{Mo} d}\right|^{2}$ & 24 & 15 & 17 & 11 & 7 \\
\hline$\frac{1}{30}\left|S_{X d}^{\mathrm{Mo} d}\right|^{2}$ & 75 & 43 & 53 & 31 & 19 \\
\hline$P_{\text {Mo } p}\left(C_{\text {Mo } d}\right)$ & -18 & -18 & -18 & -18 & -18 \\
\hline$P_{X s}\left(C_{\text {Mo } d}\right)$ & 2.7 & 2.7 & 2.7 & 2.3 & 2.1 \\
\hline$P_{X p}\left(C_{\mathrm{Mo} d}\right)$ & 4.5 & 3.2 & 3.9 & 2.9 & 2.0 \\
\hline$P_{X d}\left(C_{\mathrm{Mo} d}\right)$ & -85 & -42 & -64 & -30 & -17 \\
\hline$\left.\frac{1}{30}\left|S_{\text {Mo }}^{\text {Mo }}\right|^{d}\right|^{2} / P_{\text {Mo } p}\left(C_{\text {Mo } d}\right)$ & -0.4 & -0.4 & -0.4 & -0.4 & -0.4 \\
\hline$\frac{1}{30}\left|S_{X s}^{\mathrm{Mo} d}\right|^{2} / P_{X s}\left(C_{\mathrm{Mo} d}\right)$ & 0.5 & 0.4 & 0.4 & 0.3 & 0.3 \\
\hline$\frac{1}{30}\left|S_{X p}^{M o d}\right|^{2} / P_{X p}\left(C_{M o d}\right)$ & 5.3 & 4.7 & 4.4 & 3.8 & 3.5 \\
\hline$\frac{1}{30}\left|S_{X d}^{\mathrm{Mo} d}\right|^{2} / P_{X d}\left(C_{\mathrm{Mod} d}\right)$ & -0.9 & -1.0 & -0.8 & -1.0 & -1.2 \\
\hline$\frac{1}{30}\left|S_{\text {Mo } p}^{\text {Mo } d}\right|^{2} \Delta_{\text {Mo } d} \Delta_{\text {Mo } p}\left(C_{\text {Mo } d}-C_{\text {Mo }} d^{-2}\right.$ & 0.04 & 0.04 & 0.04 & 0.04 & 0.04 \\
\hline$\frac{1}{30}\left|S_{X s}^{\mathrm{Mod} d}\right|^{2} \Delta_{\text {Mo } d} \Delta_{X s}\left(C_{\text {Mio } d}-C_{X s}\right)^{-2}$ & 0.003 & 0.003 & 0.002 & 0.003 & 0.002 \\
\hline$\frac{1}{30}\left|S_{X p}^{\mathrm{Mo} d}\right|^{2} \Delta_{\mathrm{Mo} d} \Delta_{X p}\left(C_{\mathrm{Mo} d}-C_{X p}\right)^{-2}$ & 0.43 & 0.43 & 0.40 & 0.38 & 0.42 \\
\hline$\frac{1}{30}\left|S_{X d}^{\mathrm{Mo} d}\right|^{2} \Delta_{\mathrm{Mo} d} \Delta_{X d}\left(C_{\mathrm{Mo} d}-C_{X d}\right)^{-2}$ & 0.04 & 0.04 & 0.04 & 0.04 & 0.07 \\
\hline
\end{tabular}

according to (17a); that is, $P_{\mathrm{Mo} d}=S_{\mathrm{Mo} d, j}$ $+\left|S_{X p}^{\text {Mo } d}\right|_{j}^{2} P_{X p}^{-1}$. When $\left|P_{X p}\right| \lesssim W_{X p}$ and $\left|P_{\text {Mo } d}\right| \lesssim W_{\text {Mo } d}$ the hybridization is strong and (17a) does not apply. For the average hybridization constant defined in (21) we obtain

$$
\begin{aligned}
\frac{1}{30}\left|S_{X p}^{\mathrm{Mod} d}\right|^{2} & =180\left[4\left(s / R_{\mathrm{Mo}-X}^{\mathrm{intra}}\right)^{8}+\left(s / R_{\mathrm{Mo}-X}^{\mathrm{inter}}\right)^{8}\right] \\
& =1.57\left[4+\left(\sqrt{2} R_{\mathrm{Mo}-X}^{\mathrm{inter}} / b^{\prime}\right)^{-8}\right]\left(b^{\prime} / b\right)^{-8},
\end{aligned}
$$

since each of the six Mo atoms has four nearest $X$ neighbors at the approximate distance $b^{\prime} / \sqrt{2}$ plus one neighbor at the distance $R_{\mathrm{Mo}-X}^{\mathrm{inter}}$. For the compounds in Table I, $\frac{1}{30}\left|S_{X p}^{\text {Mo } d}\right|^{2}=24,15,17,11$, and 7 , which reflect the trend in $b^{\prime} / b$. These values are quoted in Table IV.

The differences between the canonical eigenvalue surfaces shown in Figs. 9(a)-9(c) therefore have two major causes: The Mo $d-X p$ mixing is stronger in $\mathrm{Mo}_{6} \mathrm{~S}_{8}$ than in $\mathrm{Mo}_{6} \mathrm{Se}_{8}$ and $\mathrm{PbMo}_{6} \mathrm{~S}_{7.5}$, because the Mo- $X$ distances are relatively smaller, and the splittings of the levels of the perfect octahedron are larger in the binaries than in $\mathrm{PbMo}_{6} \mathrm{~S}_{7.5}$ because the distortions $c / b$ are larger.

Although (17a) only holds at the extreme righthand sides of Fig. 9 and we neglect the influence of the Mo $s$ waves, we may understand the changes in ordering of the Mo $d$-like levels found from the full calculations and shown in the figure by considering how the hybridization constant $\left|S_{X p}^{\mathrm{Mo} d}\right|_{j}^{2}$ differs for various Mo $d$ states $j$. For simplicity we shall assume that the six Mo atoms and the eight $X$ atoms of the same cell are placed at, respectively, the face centers and the corners of the $b^{\prime}$ cube, and that the six $X$ atoms in the neighboring cells are placed at the positions $\left(0,0, R_{\mathrm{Mo}-X}^{\mathrm{inter}}+\frac{1}{2} b^{\prime}\right)$, etc., i.e., that $n_{\mathrm{Mo}-X}^{\text {inter }}=1$. (See text to Fig. 1 and Table I.) Moreover, we shall use the values of $b^{\prime}$ and $R_{\text {Mo-X }}^{\text {inter }}$ appropriate for $\mathrm{PbMO}_{6} \mathrm{~S}_{7.5}$ and consider Fig. 9(d).

For the $A_{1 g}$ state we find from (18a) that

$$
\begin{aligned}
\left|S_{X p}^{\mathrm{Mo} d}\right|_{j}^{2}= & \frac{8}{6}\left[\mid S\left(z, 3 z^{2}-r^{2} ;-\frac{1}{2} b^{\prime}, \frac{1}{2} b^{\prime}, 0\right)\right. \\
& \left.+S\left(z, 3 x^{2}-r^{2} ; 0, \frac{1}{2} b^{\prime}, \frac{1}{2} b^{\prime}\right)+\left.S\left(z, 3 y^{2}-r^{2} ;-\frac{1}{2} b^{\prime}, 0, \frac{1}{2} b^{\prime}\right)\right|^{2}+|S(y, \ldots) \ldots|^{2}+|S(x, \ldots) \ldots|^{2}\right] \\
& +\frac{6}{6}\left[\left|S\left(z, 3 z^{2}-r^{2} ; 0,0, R_{\mathrm{Mo}-X}^{\text {inter }}\right)\right|^{2}+|S(y, \ldots) \ldots|^{2}+|S(x, \ldots) \ldots|^{2}\right] \\
& =\frac{8}{6}\left(3\left|0+\sqrt{\frac{3}{8}}+\sqrt{\frac{3}{8}}\right|^{2}\right) 180\left(s / R_{\mathrm{Mo}-X}^{\text {intra }}\right)^{8}+\frac{6}{6}\left(|\sqrt{3}|^{2}+0+0\right) 180\left(s / R_{\mathrm{Mo}-X}^{\text {inter }}\right)^{8}=22+7=29 .
\end{aligned}
$$


The factors 8 and 6 are the respective numbers of $X$ atoms and the factor $\frac{1}{6}$ derives from the normalization of the $A_{1 g}$ wave function. The value of this hybridization constant is larger than the average value of 17 listed in Table IV.

The mixing of the $x^{2}-y^{2}$ and $x y$ Mo orbitals with the $p$ orbitals at the $X$ site in the neighbor cell is very small, and it vanishes for $n_{\mathrm{Mo}-X}^{\text {inter }}=1$ because the $d$ orbitals considered have $|m|=2$ about the $R_{\mathrm{Mo}-X}^{\mathrm{inter}}$ axis. For the $A_{2 u}$ state,

$$
\begin{aligned}
\left|S_{X p}^{M o} d\right|_{j}^{2}=\frac{8}{6} & {\left[3 \mid S\left(z,-x y ;-\frac{1}{2} b^{\prime}, \frac{1}{2} b^{\prime}, 0\right)\right.} \\
& +S\left(z, z y ; 0, \frac{1}{2} b^{\prime}, \frac{1}{2} b^{\prime}\right) \\
& \left.+\left.S\left(z,-z x ; \frac{1}{2} b^{\prime}, 0, \frac{1}{2} b^{\prime}\right)\right|^{2}\right] \\
& =\frac{8}{6}\left(3|0-3 / \sqrt{8}-3 / \sqrt{8}|^{2}\right) 180\left(s / R_{\text {Mo-X }}^{\text {intra }}\right)^{8}=65 .
\end{aligned}
$$

This pure intracluster mixing, which is three times that of the $A_{1 g}$ state, shifts the $A_{2 u}$ level far above the Fermi level and thereby contributes to the binding of the $\mathrm{Mo}_{6} X_{8}$ cluster.

Most crucial is the inversion of the lower $E_{g}$ and $T_{2 u}$ levels. From the full calculations for $\mathrm{PbMo}_{6} \mathrm{~S}_{7_{05}}$ it appeared that the probabilities in an $E_{g}$ state of the Mo $x^{2}-y^{2}$, Mo $3 z^{2}-r^{2}$, Mo $s$, and intracell $X p$ waves were $0.85,0.005,0.005$, and 0.14 respectively. This confirms that, for the $E_{g}$ level, the intercell $X p$ mixing is vanishingly small and that the Mo $3 z^{2}-r^{2}$ content is even smaller than the value $0.21^{2}=0.04$ found in Sec. III for the pure Mo $d$ state. Neglecting therefore the $3 z^{2}-r^{2}$ content, we find for the hybridization constant of the $E_{g}$ state, shown in Fig. 4,

$$
\begin{aligned}
\left|S_{X p}^{\mathrm{Mod} d}\right|_{j}^{2} & =\frac{8}{6}\left[3 \mid S\left(x, x^{2}-y^{2} ;-\frac{1}{2} b^{\prime},-\frac{1}{2} b^{\prime}, 0\right)\right. \\
& +\exp \left(\frac{2}{3} \pi i\right) S\left(x, y^{2}-z^{2} ; 0,-\frac{1}{2} b^{\prime},-\frac{1}{2} b^{\prime}\right) \\
& \left.+\left.\exp \left(-\frac{2}{3} \pi i\right) S\left(x, z^{2}-x^{2} ;-\frac{1}{2} b^{\prime}, 0,-\frac{1}{2} b^{\prime}\right)\right|^{2}\right] \\
& =\frac{8}{6}\left[3\left|(-1 / \sqrt{2})+0+\exp \left(-\frac{2}{3} \pi i\right)(1 / \sqrt{2})\right|^{2}\right] \\
& \times 180\left(s / R_{\text {Mo-X }}^{\text {intra }}\right)^{8}=22 .
\end{aligned}
$$

For the $T_{2 u}$ state shown in Fig. 5 we find, neglecting for the moment the $(x z, y z)$ content,

$$
\begin{aligned}
\left|S_{X p}^{\text {Mo } d}\right|_{j}^{2}=\frac{8}{2} & {\left[\left|S\left(x, x^{2}-y^{2} ;-\frac{1}{2} b^{\prime},-\frac{1}{2} b^{\prime}, 0\right)\right|^{2}\right.} \\
& +\left|S\left(y, x^{2}-y^{2} ;-\frac{1}{2} b^{\prime},-\frac{1}{2} b^{\prime}, 0\right)\right|^{2} \\
& \left.+\left|S\left(z, x^{2}-y^{2} ;-\frac{1}{2} b^{\prime},-\frac{1}{2} b^{\prime}, 0\right)\right|^{2}\right] \\
& =\frac{8}{2}\left[(-1 / \sqrt{2})^{2}+(1 / \sqrt{2})^{2}+0\right] 180\left(s / R_{\text {Mo-X }}^{\text {in tra }}\right)^{8}=15,
\end{aligned}
$$

which is two-thirds the hybridization of the $E_{g}$ state. Including the mixing with the $x z$ and $y z$ orbitals, we find

$$
\begin{aligned}
\left|S_{X p}^{\text {Mo d d }}\right|_{j}^{2}= & \frac{8}{2}\left(1+\beta^{2}\right)^{-1} 2 \mid S\left(x, x^{2}-y^{2} ;-\frac{1}{2} b^{\prime},-\frac{1}{2} b^{\prime}, 0\right) \\
& +(\beta / \sqrt{2}) S\left(x,-z x ; 0,-\frac{1}{2} b^{\prime},-\frac{1}{2} b^{\prime}\right) \\
& +\left.(\beta / \sqrt{2}) S\left(x, z y ;-\frac{1}{2} b^{\prime}, 0,-\frac{1}{2} b^{\prime}\right)\right|^{2} \\
& +\beta^{2}\left(1+\beta^{2}\right)^{-1}\left|S\left(z, z y ; 0,-R_{\mathrm{Mo}-X}^{\text {inter }}, 0\right)\right|^{2} \\
& =\frac{8}{2}(1-\beta / \sqrt{2})^{2}\left(1+\beta^{2}\right)^{-1} 180\left(s / R_{\mathrm{Mo}-X}^{\text {intra }}\right)^{8} \\
& +\beta^{2}\left(1+\beta^{2}\right)^{-1} 180\left(s / R_{\text {Mo-X }}^{\text {inter }}\right)^{8}=3.2+0.7=4,
\end{aligned}
$$

using the value $\beta=0.63$ which applies to the pure Mo $d$ state and corresponds to the occupancy $\beta^{2}\left(1+\beta^{2}\right)^{-1}=0.29$. This strong mixing with the $x z$ and $y z$ orbitals therefore reduces the $X p$ covalent mixing of the $T_{2 u}$ state to a value which is only about one-fifth of the value in the $E_{g}$ state.

In $\mathrm{PbMo}_{6} \mathrm{~S}_{7.5}$ the $E_{g}$ level will therefore lie above the $T_{2 u}$ level when $P_{X p}$ is smaller than the value determined by $-3.1+22 P_{X p}^{-1}=-1.7+4 P_{X p}^{-1}$, that is, when $P_{X p}<13$. This is in accord with the calculations illustrated in Figs. 9(c) and (d). For the series $\mathrm{Mo}_{8} \mathrm{~S}_{8}, \mathrm{Mo}_{6} \mathrm{Se}_{8}, \mathrm{PbMo}_{6} \mathrm{~S}_{7.5}, \mathrm{PbMo}_{6} \mathrm{Se}_{8}$, and $\mathrm{Mo}_{6} \mathrm{Te}_{8}$, we estimate that the $E_{g}$ level will lie above the $T_{2 u}$ level, i.e., the center of gravity of the $E_{u}$ and $A_{u}$ levels, when $P_{X p}$ is less than, respectively, $17,11,13,8$, and 6 , because $\left|S_{X p}^{\mathrm{Mo} d}\right|^{2}$ is proportional to $\left(b^{\prime} / b\right)^{-8}$. At the estimated potential paths and including now the $E_{u}-A_{u}$ splitting, we conclude that: In $\mathrm{Mo}_{6} \mathrm{~S}_{8}$ and $\mathrm{Mo}_{6} \mathrm{Se}_{8}$ the $A_{u}$ and $E_{g}$ levels are nearly degenerate, in $\mathrm{Mo}_{6} \mathrm{Te}_{8}$ the $E_{g}$ level lies between the $E_{u}$ and $A_{u}$ levels, and in $\mathrm{PbMo}_{6} \mathrm{~S}_{7.5}$ and $\mathrm{PbMo}_{6} \mathrm{Se}_{8}$, where the $E_{u}-A_{u}$ splitting is small, the $E_{g}$ level lies above both levels.

For the $A_{2 g}$ state,

$$
\begin{aligned}
\left|S_{X p}^{M_{0} d}\right|_{j}^{2}=\frac{8}{6} & {\left[3 \mid S\left(x, x^{2}-y^{2} ;-\frac{1}{2} b^{\prime},-\frac{1}{2} b^{\prime}, 0\right)\right.} \\
& +S\left(x, y^{2}-z^{2} ; 0,-\frac{1}{2} b^{\prime},-\frac{1}{2} b^{\prime}\right) \\
& \left.+\left.S\left(x, z^{2}-x^{2} ;-\frac{1}{2} \cdot b^{\prime}, 0,-\frac{1}{2} b^{\prime}\right)\right|^{2}\right]=0 .
\end{aligned}
$$

This is clearly seen in Fig. 9.

The Mo $s$-like levels which transform according to $A_{1 g}, T_{2 u}\left(\rightarrow A_{u}+E_{u}\right)$, and $E_{g}$ may be identified by comparison of Fig, 9 (c) for $\mathrm{PbMo}_{6} \mathrm{~S}_{7.5}$ with Fig. $9(\mathrm{~d})$, in which the plane of cross section with the eigenvalue surfaces has been shifted from $P_{\text {Mo } d}$ $-5 P_{\text {Mo } s}=-6.25$ to $P_{\text {Mo } d}-5 P_{\text {Mo } s}=0$. The fact that the levels labeled $T_{2 u}(s)$ and $E_{g}(s)$ shift upwards by about $6.25(\approx 220 \mathrm{mRy})$ indicates that these eigenvalue surfaces are almost perpendicular to the $P_{\text {Mo } s}$ axis and, according to (6), they are therefore Mo $s$-like. In Fig. 10 we show for $\mathrm{PbMo}_{6} \mathrm{~S}_{7.5}$ the cross sections with the plane $P_{\text {Mo } d}$ $-5 P_{X p}=-15$, which is parallel with the $P_{M o s}$ axis and cuts the planes of Figs. 9(c) and 9(d) along lines rather close to our estimated potential path. The path is seen to be almost parallel to the $T_{2 u}(s)$ and $E_{g}(s)$ levels and the corresponding ener- 
gies will therefore presumably lie well above the Mo $d$ energies in ali the molybdenum chalcogenides. When $P_{M o d}$ and $P_{X p} \rightarrow \infty$ the $T_{2 u}(s)$ and $E_{g}(s)$ states will tend towards the pure Mo $s$ states for the isolated octahedron. These are, respectively,

$\left\{s_{z}-s_{-z}, s_{x}-s_{-x}, s_{y}-s_{-y}\right\}$

and

$\left(s_{z}+s_{-z}\right)+\exp \left(\mp \frac{2}{3} \pi i\right)\left(s_{x}+s_{-x}\right)+\exp \left( \pm \frac{2}{3} \pi i\right)\left(s_{y}+s_{-y}\right)$

and have the respective energies

$-S(s, s ; 0,0, b)=2(s / b)=0.782$

and

$$
\begin{aligned}
-2 S\left(s, s ; \frac{1}{2} b, \frac{1}{2} b, 0\right)+S(s, s ; 0,0, b) & =2(2 \sqrt{2}-1)(s / b) \\
& =1.428
\end{aligned}
$$

for a perfect octahedron.

Figure 10 shows that, whereas the states labeled $T_{2 u}(s)$ and $E_{g}(s)$ do not hybridize significantly with the $d$-like $E_{g}$ and $T_{2 u}$ states in the range of interest (but rather with some $p$-like levels lying below the range shown in the figure), the previously considered $A_{1 g}$ state hybridizes strongly with a lower-lying $A_{1 g}$ state, labeled $A_{1 g}^{\prime}$. At the potential path, the Mo $d$, Mo $s$, and $X p$ probabilities of the $A_{1 g}$ state are, respectively, $0.46,0.09$, and 0.45 , and the corresponding values for the lower-lying $A_{1 g}^{\prime}$ states are $0.14,0.33$, and 0.53 . When $P_{\text {Mo } d}$ and $P_{X p} \rightarrow \infty$ the $A_{1 g}$ state tends towards the pure Mo $s$ state $s_{x}+s_{-x}+s_{y}+s_{-y}+s_{z}+s_{-z}$ with the energy

$$
\begin{aligned}
4 S\left(s, s ; \frac{1}{2} b, \frac{1}{2} b, 0\right)+S(s, s ; 0,0, b) & =-2(4 \sqrt{2}+1)(s / b) \\
& =-5.20,
\end{aligned}
$$

and when $P_{\text {Mo } s} \rightarrow \infty$ this $A_{1 g}$ level tends towards a $d p$-like level. The $A_{1 g}^{\prime}$ level tends towards the same $d p$-like level when $P_{\text {Mo } s} \rightarrow-\infty$ and towards a $p d$-like level when $P_{\text {Mo } s} \rightarrow \infty$. Finally, below the range of the figure, there are two further $A_{1 g}$ levels of which the lowest tends towards the pure $s$ level at -5.20 when $P_{\text {Mo } d}$ and $P_{X p} \rightarrow-\infty$.

Since only five of the six Mo $s$-like levels are above the Fermi level, the latter will be placed between the $E_{u}$ and $E_{g}$ levels in the binaries, which have 20 Mo electrons per cell, between the $A_{u}$ and $E_{g}$ levels in $\mathrm{PbMo}_{6} \mathrm{Se}_{8}$, having 22 Mo electrons per cell, and in the $E_{g}$ level in $\mathrm{PbMo}_{6} \mathrm{~S}_{7.5}$, which has 23 Mo electrons per cell. It may, however, well be beyond the accuracy of a cluster model to decide whether the position of the uppermost $A_{1 g}$ band in the crystal is above or below that of the $E_{g}$ band. If the $A_{1 g}$ band is empty, the Fermi level will be placed in the $E_{g}$ band for all the compounds considered.

This uncertainty, which stems from the long range of the Mo $s$ orbitals (see Sec. IIC), only applies to levels with some Mo $s$ character and, as seen from Fig. 10, these include the $A_{1 g}$ levels [and the empty $E_{g}(s)$ and $T_{2 u}(s)$ levels] only. Had we increased the size of our model by including the $s$ waves in the $14 X$ spheres, this would, in the region of interest well above the $X s$ levels, have had the effect of moving the constant energy surfaces in the positive $P_{\text {Mo } s}$ direction or, equivalently, of moving the potential path to the left in Fig. 10. An upper bound on this shift is

$$
\begin{aligned}
\frac{1}{30}\left|S_{X s}^{\mathrm{Mo}} s\right|^{2} /\left[P_{X s}\left(C_{\text {Mo } d}\right)-S_{X s}\right] & =2.1 /(2.72-2.22) \\
& =5,
\end{aligned}
$$

where we have used the average Mo $s-X s$ hybridization constant (22) and the structure constant 2.22, valid for the uppermost $X s$ level. [On energy scale and for $\mathrm{PbMo}_{6} \mathrm{~S}_{7.5}$, the top of the $X s$ band is 0.73 Ry below $C_{\text {Mo } d}$; i.e., $P_{X s}(-0.73)$ $=2.22$.] With the maximum shift of the potential path, as indicated in Fig. 10, all six Mo s-like levels would be well above the Mo $d$ band. If we then increased the size of the cluster by adding more Mo atoms, the position of the Mo $s$ bands would again drop a bit, and it is obvious that a satisfactory description of the Mo $s$-like bands can only be obtained in a full band calculation, employing the Ewald summation for the structure constants of low $l+l^{\prime}$.

In the neighborhood of the estimated potential paths, and near the Fermi levels, the Mo $d$ states may hybridize strongly only with the $X p$ and the Mo $s$ states because all other bands lie far outside the range of the Mo $d$ band. This can be seen from the values of the potential parameters listed in Table III. The influence on the Mo $d$ levels of the so far neglected Mo $p, X s$, and $X d$ partial waves has been estimated in Table IV, where we list the average hybridization constants (22), the values of the potential functions at the center of the Mo $d$ band, (11), the average hybridization shifts (21), and the corresponding partial probabilities (20). The hybridization shifts neglected in Figs. 9 and 10 are thus seen to be less than $\left|\delta P_{\text {Mod }}\right|=1$ on the average, and we expect the shifts between the individual Mo $d$-like levels, which are the only shifts of significance, to be somewhat smaller. The neglected partial probabilities are seen to be less than 0.1 . In view of the approximate character of the present calculations we conclude that the effect of these partial waves on the Mo $d$-subband positions is negligible. In Sec. IV C we shall see that the effect of the $X d$ waves on the Mo $d$-subband widths may nevertheless be significant. 


\section{B. Self-consistency}

Average quantities referring to the covalent mixing with the $X p$ band have been listed in Table IV too, and although the weak-hybridization expressions (20) and (21) are not strictly applicable in this case, they yield reasonable estimates because the pure $X p$ and Mo $d$ bands do not overlap. We shall now use the partial probabilities (20) to explain the self-consistent values of the Mo $d-X p$ band separation $C \equiv C_{\mathrm{Mo} d}-C_{X p}$ found from the full cluster calculations and given in $\mathrm{Ta}-$ ble III.

For simplicity we shall assume that the conduction-electron density in a Mo sphere arises solely from the Mo $d$ waves and that the valence-electron density in an $X$ sphere arises solely from the $X p$ waves. Since all $X p$ bands are full, these contribute

$$
2 \Delta_{\text {Mo } d}\left|S_{X p}^{\mathrm{Mo} d}\right|^{2} \Delta_{X p}\left(C_{\text {Mo } d}-C_{X p}\right)^{-2} \equiv 2 H^{2} / C^{2}
$$

electrons to the $h=6$ Mo spheres. A neutral Mo atom has six conduction electrons, and if $q$ is the oxydation state of Mo in the compound, there are $\frac{1}{2}(6-q) h=10,11$, and 11.5 occupied Mo $d$ bands in $\mathrm{Mo}_{6} X_{8}, M \mathrm{Mo}_{6} X_{8}$, and $M \mathrm{MO}_{6} X_{7.5}$, respectively. These subbands contribute $(6-q) h\left[1-h(\text { Mo } d)^{-1} H^{2} /\right.$ $\left.C^{2}\right]$ electrons to the Mo spheres, in the approximation that we substitute $\left|S_{X p}^{\mathrm{Mo} d}\right|_{j}^{2}$ in (20) by its average, $h(\text { Mo } d)^{-1}\left|S_{X p}^{\text {Mod } d}\right|^{2}$. The charge per Mo sphere is therefore $Q=q-2 h^{-1}(1-f) H^{2} / C^{2}$, where $f \equiv \frac{1}{10}(6-q)$ is the fractional occupancy of the Mo $d$ band. If there were no charge transfer, the band separation would be

$$
\begin{aligned}
C^{b} & \equiv C_{\text {Mo } d}^{b}-C_{X p}^{b} \\
& =\left(\frac{2(1-f)}{q h} \Delta_{\text {Mo } d}\left|S_{X p}^{\text {Mo } d}\right|^{2} \Delta_{X p}\right)^{1 / 2} \\
& \equiv\left(\frac{2(1-f)}{q h}\right)^{1 / 2} H,
\end{aligned}
$$

and the relation between $Q$ and $C$ provided by the band structure may conveniently be written as

$$
C / C^{b}=(1-Q / q)^{-1 / 2} \approx 1+\frac{1}{2} Q / q,
$$

where the approximation is valid if $|Q / q| \ll 1$.

From Tables III and IV, and for the usual sequence of compounds, we find $H=1.38,1.13,1.07$, 0.97 , and $0.90 \mathrm{Ry}$ and hence $C^{b}=0.37,0.33,0.32$, 0.29 , and $0.26 \mathrm{Ry}$. (For the nonstoichiometric compound $\mathrm{PbMo}_{6} \mathrm{~S}_{7.5}$ we have multiplied $\left|S_{X p}^{\mathrm{Mo} d}\right|^{2}$ by 7.5/8.)

The band positions $C_{\text {Mo } d}^{a}$ and $C_{X p}^{a}$ obtained from the neutral, renormalized Mo and $X$ atom potentials yield $C_{\text {Mo } d}^{a}-C_{X p}^{a} \approx 0.15,0.20,0.25,0.30$, and $0.25 \mathrm{Ry}$. If we bring the extra electronic charge $-Q_{\text {Mo }}\left(-Q_{X}\right)$ into the Mo $(X)$ sphere, $C_{\text {Mo } d}\left(C_{X p}\right)$ is raised by $-Q_{\mathrm{Mo}} u_{\mathrm{Mo} d}\left(-Q_{X} u_{X p}\right)$, where it turns out that $u_{\mathrm{Mo} d} \approx u_{\mathrm{Xp}} \approx 0.8 \mathrm{Ry}$. Charge neutrality requires $6 Q_{\mathrm{MO}}+8 Q_{X}+Q_{M}=0$, where $Q_{\mathrm{MO}} \equiv Q$ and $Q_{M}=0$ or 2 , and the Coulomb repulsion, reduced by exchangecorrelation and including the Madelung shifts, therefore gives the relation

$$
C=C^{a}-Q u,
$$

where $u \approx 1$ Ry and $C^{a} \approx C_{M 0 d}^{a}-C_{X p}^{a}-\frac{1}{8} Q_{M} u_{X p} \approx 0.15$, $0.20,0.05,0.10$, and $0.25 \mathrm{Ry}$.

The band separation $C$ and the charge transfer $Q$ may now be obtained from (28) and (29). When $\left|\left(C^{a}-C^{b}\right) / 2 u q\right| \ll 1$, we find $Q \approx\left(C^{a}-C^{b}\right) / u$ and hence $|Q / q| \ll 1$. In this case,

$$
C \approx C^{b}\left(1+\frac{C^{a}-C^{b}}{2 u q}\right)
$$

so that the band separation is determined by the hybridization rather than by the Coulomb interaction or, in other words, covalency dominates over ionicity. For the compounds considered, $\left(C^{a}-C^{b}\right) /$ $2 u q \approx-0.04,-0.02,-0.06,-0.04$, and 0 . The charge transfer is therefore small, $-0.3 \lesssim Q \lesssim 0$, and the band separation is only a few percent smaller than that, $C^{b}$, determined from the band structure alone. Specifically, $C=0.35,0.32,0.30$, 0.28 , and $0.26 \mathrm{Ry}$, and our estimates of $C^{a}$ and $u$ need only be approximate.

The values of $C$ thus determined, neglecting the Mo $s$ waves and assuming the Mo $d-X p$ mixing to be weak, agree fortuitously well with those obtained from the full cluster calculations and given in Table III. The reason is that the weak-hybridization expression (20) overestimates the Mo $d-X p$ covalent mixing and thereby effectively accounts for the strong Mo $s-X p$ mixing.

\section{Band states}

The cluster levels in Fig. 9 give the positions of the Mo $d$-like bands in $M_{m} \mathrm{Mo}_{6} X_{8-x}$ crystals because all the nearest neighbors of a given octahedron are included in the $\mathrm{Mo}_{6} \mathrm{X}_{14}$ cluster and, for $d$-like bands, only the near-neighbor interactions are important. The band broadening was studied in Sec. III B, where we estimated the direct term $S_{\mathrm{Mo} d}^{\text {inter }}$ of the intercluster coupling. For a given translation vector $\overrightarrow{\mathrm{T}}$, e.g., the one in the negative $k$ direction (see Fig. 1), this is the $d d$ interaction between the Mo atoms at the sites $\left(0,0, \frac{1}{2} b\right)+\vec{T}$ and $\left(0,0,-\frac{1}{2} b\right)$. Using the approximation of weak hybridization, (17b) and (18b), we now calculate the indirect interactions $\left(S_{X l}^{\mathrm{Mo} d}, \overrightarrow{\mathrm{T}} S_{\mathrm{Mo} d}^{X l}\right)_{j} P_{X l}^{-1}$ and $\left(S_{X i, \vec{T}}^{\mathrm{Mo}} S_{\mathrm{Mo}}^{X}{ }_{d, \overrightarrow{\mathrm{T}}}\right)_{j} P_{X l}^{-1}$. Of these equivalent interactions the first corresponds to a bond from the Mo atom at $\left(0,0, \frac{1}{2} b\right)+\overrightarrow{\mathrm{T}}$, via the $X$ atom at $\frac{1}{2}(1,-1,-1) b^{\prime}$, and to the three Mo atoms at, respectively, 
$\left(0,0,-\frac{1}{2} b\right),\left(0,-\frac{1}{2} b, 0\right)$ and $\left(\frac{1}{2} b, 0,0\right)$. The sum of these interactions may be interpreted as the $X l$ contribution to the three-center integral for the Mo $d$ Bloch state $j$. Since, at the $X$ site considered, there is no overlap from any third octahedron, the Mo $d$-like bands are still expressed by (24) with $G(\overrightarrow{\mathrm{k}})$ unchanged, with $S^{\text {intra }}$ equal to the respective cluster level in Fig. 9, and with

$$
S^{\mathrm{inter}}=S_{\mathrm{Mo} d}^{\mathrm{inter}}+\sum_{t l \neq \mathrm{Mo} d} 2 \operatorname{Re}\left(S_{t l}^{\mathrm{Mo} d,} \overrightarrow{\mathrm{T}}^{t} S_{\mathrm{Mo} d}^{t l}\right)_{j} P_{t l}^{-1}
$$

In this expression also the intercluster hybridization with the Mo $s$ and $p$ waves has been included.
Of the corresponding two equivalent bonds one goes from the Mo $d$ orbital at $\left(0,0, \frac{1}{2} b\right)+\overrightarrow{\mathrm{T}}$, via the Mo $s$ and $p$ waves at $\left(0,0,-\frac{1}{2} b\right)$, and to the four Mo $d$ orbitals at $\left( \pm \frac{1}{2} b, 0,0\right)$ and $\left(0, \pm \frac{1}{2} b, 0\right)$.

If it were exactly true that $l_{\mathrm{Mo}-X}^{\text {inter }}=m_{\mathrm{Mo}-X}^{\text {inter }}=0$ (see text to Fig. 1 and Table I), the $X s$ and $X p$ intercluster contribution to the $E_{g}$ states would vanish as mentioned in Sec. IV A. For the actual values of $l_{\text {Mo-X }}^{\text {inter }}$ and $m_{\text {Mo-X }}^{\text {inter }}$ given in Table $\mathrm{I}$, the $X s$ mixing may still be neglected, but owing to the proximity of the $X p$ band and the correspondingly small value of $P_{X p}$ (Table IV), the broadening of the $E_{g}$ band caused by $X p$ intercluster mixing is significant. To first order in $l_{\mathrm{Mo}-X}^{\text {inter }}$, we specifically find

$$
\begin{aligned}
& 2 \operatorname{Re}\left(S_{X p}^{\mathrm{Mo} d, \overrightarrow{\mathrm{T}}} S_{\mathrm{Mo} d}^{X p}\right)_{j} \\
& =2 \operatorname{Re} S\left(x^{2}-y^{2}, x ; \overrightarrow{\mathrm{R}}_{\mathrm{Mo}-X}^{\mathrm{inter}}\right)\left[S\left(x, z^{2}-x^{2} ;-\frac{1}{2} b^{\prime}, 0, \frac{1}{2} b^{\prime}\right) \exp \left(-\frac{2}{3} \pi i\right)+S\left(x, x^{2}-y^{2},-\frac{1}{2} b^{\prime}, \frac{1}{2} b^{\prime}, 0\right)\right] \\
& =3 S\left(x^{2}-y^{2}, x ; \overrightarrow{\mathrm{R}}_{\mathrm{Mo}-\mathrm{X}}^{\mathrm{inter}}\right) S\left(x, x^{2}-y^{2} ;-\frac{1}{2} b^{\prime}, \frac{1}{2} b^{\prime}, 0\right) \\
& =3\left(l_{\text {Mo-X }}^{\text {inter }} / \sqrt{2}\right) 180\left(s / R_{\text {Mo-X }}^{\text {inter }}\right)^{4}\left(s / R_{\text {Mo-X }}^{\text {intra }}\right)^{4} \\
& =3.32 l_{\mathrm{Mo}-X}^{\text {inter }}\left(b^{\prime} / b\right)^{-8}\left(\sqrt{2} R_{\mathrm{Mo}-X}^{\text {inter }} / b^{\prime}\right)^{-4} \\
& =1.06,0.82,0.90,0.67 \text {, and } 0.39 \text {. }
\end{aligned}
$$

\begin{tabular}{|c|c|c|c|c|c|}
\hline & $\mathrm{Mo}_{6} \mathrm{~S}_{8}$ & $\mathrm{Mo}_{6} \mathrm{Se}_{8}$ & $\mathrm{PbMo}_{6} \mathrm{~S}_{7.5}$ & $\mathrm{PbMo}_{6} \mathrm{Se}_{8}$ & $\mathrm{Mo}_{6} \mathrm{Te}_{8}$ \\
\hline \multicolumn{6}{|c|}{$E_{g}\left(x^{2}-y^{2}\right)$} \\
\hline$S_{\text {Mo d }}^{\text {inter }}$ & 0.53 & 0.31 & 0.28 & 0.18 & 0.14 \\
\hline $2 \operatorname{Re}\left(S_{X p}^{M o d}, \overrightarrow{\mathrm{T}} S_{M 0 d}^{X p}\right) / P_{X p}$ & 0.23 & 0.26 & 0.23 & 0.23 & 0.20 \\
\hline $2 \operatorname{Re}\left(S_{X d}^{\mathrm{Mo} d}, \overrightarrow{\mathrm{T}}_{S_{\mathbf{M O} d}^{X d}}\right) / P_{X d}$ & 0.14 & 0.15 & 0.11 & 0.14 & 0.15 \\
\hline$S^{\text {inter }}$ (total) & 0.90 & 0.72 & 0.62 & 0.55 & 0.49 \\
\hline Bandwidth (mRy) & 65 & 50 & 45 & 40 & 35 \\
\hline$N(0)[$ states/(spin Mo-atom Ry)] & & 7 & 11 & 8 & \\
\hline \multicolumn{6}{|c|}{$A_{u}\left[x^{2}-y^{2}+\beta(x-y) z / \sqrt{2}\right]$} \\
\hline$S_{\text {Mo } d}^{\text {inter }}$ & $\approx 0.1$ & $\approx 0.1$ & & & $\approx 0.1$ \\
\hline $2 \operatorname{Re}\left(S_{X p}^{\mathrm{Mod} d}, \overrightarrow{\mathrm{T}}_{S_{\mathrm{Mo} d}^{X p}}\right) / P_{X p}$ & 0.5 & 0.4 & & & 0.3 \\
\hline $2 \operatorname{Re}\left(S_{X d}^{M o d} d, \overrightarrow{\mathrm{T}}_{S_{\mathrm{Mo} d}^{X d}}\right) / P_{X d}$ & $\approx 0.1$ & $\approx 0.1$ & & & $\approx 0.1$ \\
\hline$S^{\text {inter }}$ (total) & 0.7 & 0.6 & & & 0.5 \\
\hline Bandwidth (mRy) & 50 & 40 & & & 35 \\
\hline \multicolumn{6}{|c|}{$A_{1 g}\left(3 z^{2}-r^{2}\right)$} \\
\hline$S_{\text {Mo } d}^{\text {inter }}$ & 0.77 & 0.53 & 0.49 & 0.36 & 0.30 \\
\hline $2 \operatorname{Re}\left(S_{X p}^{\mathrm{Mo} d} d \overrightarrow{\mathrm{T}}_{S_{\mathrm{Mo} d}^{X p}}\right) / P_{X p}$ & 4.3 & 3.6 & 3.2 & 3.0 & 2.6 \\
\hline$S^{\text {inter }}$ (total) & 5.1 & 4.1 & 3.7 & 3.3 & 2.9 \\
\hline Bandwidth (mRy) & 360 & 290 & 260 & 230 & 200 \\
\hline
\end{tabular}

Using the values of $P_{X p}$ given in Table IV, we obtain the values for the $X p$ contribution to the intercluster coupling in the $E_{g}$ band shown in Table V. As may be seen from Table $\mathrm{V}$, the $X d$ intercluster mixing also contributes significantly to the $E_{g}$ bandwidth. ${ }^{14}$ For this interaction we may neglect $l_{\mathrm{Mo}-X}^{\text {inter }}$ and $m_{\mathrm{Mo}-X}^{\text {inter }}$, and find

TABLE V. Mo $d$-subband widths. 


$$
\begin{aligned}
& 2 \operatorname{Re}\left(S_{X d}^{\mathrm{Mo} d} d \overrightarrow{\mathrm{T}} S_{\left.\mathrm{MO}_{d}{ }^{X}\right)_{j}}=2 S\left(x^{2}-y^{2}, x^{2}-y^{2} ; 0,0, R_{\mathrm{Mo}-\mathrm{X}}^{\mathrm{inter}}\right)\left[S\left(x^{2}-y^{2}, z^{2}-x^{2} ;-\frac{1}{2} b^{\prime}, 0, \frac{1}{2} b^{\prime}\right) \exp \left(-\frac{2}{3} \pi i\right)\right.\right. \\
& +S\left(x^{2}-y^{2}, x^{2}-y^{2} ;-\frac{1}{2} b^{\prime}, \frac{1}{2} b^{\prime}, 0\right) \\
& \left.+S\left(x^{2}-y^{2}, y^{2}-z^{2} ; 0, \frac{1}{2} b^{\prime}, \frac{1}{2} b^{\prime}\right) \exp \left(\frac{2}{3} \pi i\right)\right] \\
& =-1200\left(s / R_{\mathrm{Mo}-X}^{\text {inter }}\right)^{5}\left(s / R_{\mathrm{Mo}-X}^{\text {intra }}\right)^{5} \\
& =-3.19\left(b^{\prime} / b\right)^{-10}\left(\sqrt{2} R_{M 0-X}^{\text {inter }} / b^{\prime}\right)^{-5} \\
& =-12.2,-6.3,-7.2,-4.2 \text {, and }-2.5 \text {. }
\end{aligned}
$$

The Mo $s$ intercluster hybridization may be shown to vanish and the Mo $p$ contribution is negligible. The total values of $S^{\text {inter }}$ and the $E_{g}$ bandwidths, $2 \Delta_{\text {Mo } d}\left|S^{\text {inter }}\right|$, given in Table $V$ are thus enhanced over the direct Mo $d$-Mo $d$ values by factors varying from 1.7 in $\mathrm{Mo}_{6} \mathrm{~S}_{8}$ to 3.5 in $\mathrm{Mo}_{6} \mathrm{Te}_{8}$. The $E_{8}$ bandwidth in $\mathrm{Mo}_{6} \mathrm{~S}_{8}$ is therefore only 1.8 times the width in $\mathrm{Mo}_{6} \mathrm{Te}_{8}$, although the direct Mo-Mo intercluster distances differ by a factor of 1.2.

From Fig. 9 it is seen that the $A_{u}$ level in the binaries is nearly degenerate with the $E_{g}$ level. The direct contribution to the $A_{u}$ band width was previously found to be extremely small and, for the indirect contribution, we now find

$$
\begin{aligned}
& 2 S_{X_{p}}^{M_{0} d,}{ }^{T} S_{M_{0} d}^{X p}=2\left(1+\beta^{2}\right)^{-1} S\left(\beta(x-y) z / \sqrt{2},(x-y) / \sqrt{2} ; 0,0, R_{M_{0}-X}^{\text {inter }}\right) \\
& \times\left[S\left((x-y) / \sqrt{2}, x^{2}-z^{2}+\beta(x-z) y / \sqrt{2} ;-\frac{1}{2} b^{\prime}, 0, \frac{1}{2} b^{\prime}\right)\right. \\
& \left.+S\left((x-y) / \sqrt{2}, y^{2}-z^{2}+\beta(y-z) x / \sqrt{2} ; 0, \frac{1}{2} b^{\prime}, \frac{1}{2} b^{\prime}\right)\right] \\
& =2 \beta(1-\beta / \sqrt{2})\left(1+\beta^{2}\right)^{-1} 180\left(s / R_{\mathrm{Mo}-X}^{\text {inter }}\right)^{4}\left(s / R_{\mathrm{Mo}-X}^{\text {intra }}\right)^{4} \\
& =2.2,1.4 \text {, and } 0.6
\end{aligned}
$$

in $\mathrm{Mo}_{8} \mathrm{~S}_{8}, \mathrm{Mo}_{8} \mathrm{Se}_{8}$, and $\mathrm{Mo}_{8} \mathrm{Te}_{8}$, respectively. Therefore, the indirect contribution dominates the intercluster interaction and the band widths of this singly degenerate band are nearly equal to those of the doubly degenerate $E_{g}$ band (Table V).

For the $A_{16}$ state, and with the usual approximations,

$$
\begin{aligned}
& 2 S_{X p}^{\mathrm{Mo}} d, \overrightarrow{\mathrm{T}} S_{\mathrm{Mo} d}^{X p} \\
& =2 S\left(3 z^{2}-r^{2}, z ; 0,0, R_{M_{0}-X}^{\text {inter }}\right) \\
& \times\left[S\left(z, 3 y^{2}-r^{2} ;-\frac{1}{2} b^{\prime}, 0, \frac{1}{2} b^{\prime}\right)\right. \\
& \left.+S\left(z, 3 x^{2}-r^{2} ; 0, \frac{1}{2} b^{\prime}, \frac{1}{2} b^{\prime}\right)\right] \\
& =2 \sqrt{3} \sqrt{\frac{3}{2}} 180\left(s / R_{\mathrm{Mo}-\mathrm{X}}^{\text {inter }}\right)^{4}\left(s / R_{\mathrm{Mo}-\mathrm{X}}^{\mathrm{intra}}\right)^{4} \\
& =19,12,13,8 \text {, and } 5 \text {. }
\end{aligned}
$$

The width of the $A_{1 g}$ band is therefore completely dominated by the $X p$ intercluster mixing. The $X s$ and $X d$ contributions turn out to be negligible and so is the intercluster Mo $s$ hybridization. For the latter interaction we specifically find

$$
\begin{aligned}
& 2 S_{\text {Mo } s}^{\text {Mo } d}, \overrightarrow{\mathrm{T}} S_{\text {Mo } d}^{\text {Mo } s} \\
& =2 S\left(3 z^{2}-r^{2}, s ; \overrightarrow{\mathrm{R}}_{\mathrm{Mo}-\mathrm{Mo}}^{\text {inter }}\right) \\
& \times\left[4 S\left(s, 3 x^{2}-r^{2} ; \frac{1}{2} b, 0, \frac{1}{2} b\right)\right. \\
& \left.+S\left(s, 3 z^{2}-r^{2} ; 0,0, b\right)\right] \\
& =2 \sqrt{10}\left(\sqrt{20}+\sqrt{\frac{5}{2}}\right)\left(s / R_{\mathrm{Mo}-\mathrm{Mo}}^{\text {inter }}\right)^{3}\left(s / R_{\mathrm{Mo}-\mathrm{Mo}}^{\mathrm{intra}}\right)^{3}=0.22
\end{aligned}
$$

for $\mathrm{PbMo}_{6} \mathrm{~S}_{7.5}$. With $P_{\mathrm{Mo} s}=0.5$ (see Fig. 10) and the previously found value $S_{\mathrm{Mo} s}=\mathbf{- 5 . 2}$ for the pure $A_{1 g}$ Mo $s$ level, we find the contribution to (31)

$$
2\left(S_{\text {Mo } s}^{\text {Mo } d}, \overrightarrow{\mathrm{T}} S_{\text {Mo }}^{\text {Mo }} s\right) /\left(P_{\text {Mo } s}-S_{\text {Mo } s}\right) \approx 0.04,
$$

which is clearly negligible.

At the bottom of Fig. 9 we have sketched the extents of the Mo $d$-like $A_{1 g}, E_{g}$, and $A_{u}$ subbands as just estimated and listed in Table V. The results on energy scale, that is, along the estimated potential paths, may be found in Fig. 11, where we specifically show the $\mathrm{Mo}_{6} X_{14}$ levels in the vicinity of the respective Fermi levels together with the densities of states for the Mo $d$-like $A_{1 g}$, $E_{u}$ and $A_{u}\left(T_{2 u}\right)$, and $E_{g}$ subbands, neglecting their interactions.

As explained in Sec. IV A, the uncertainty of the band positions, obtained with our finite-size cluster model, can be expressed as an uncertainty in the position of the potential path and, for $\mathrm{PbMo}_{6} \mathrm{~S}_{7.5}$, it is given by the two limiting paths indicated in Fig. 10 by the solid and the dot-dashed curves. Only the positions of the $A_{1 g}$ bands are uncertain, and the two limiting $A_{1 g}$ densities of states are shown in Fig. 11. Going from one limit to the other, we realize that the uppermost $A_{1 g}$ band starts off Mo $d$-like, occupied with 1.4 electrons per cell. It then rises, empties its electrons into the $E_{g}\left(E_{u}\right.$ or $\left.A_{u}\right)$ bands, and becomes 
Mo $s$-like. Its role as the Mo $d$-like $A_{1 g}$ band is taken over by the $A_{1 g}^{\prime}$ band, which eventually rises to about the point when its top reaches the Fermi level. The associated uncertainties in the Fermi levels are also indicated in Fig. 11. We now summarize the conclusions which may be read from this figure.

In $\mathrm{PbMo}_{6} \mathrm{~S}_{7.5}$ and $\mathrm{PbMo}_{6} \mathrm{Se}_{8}$, where the distortion of the octahedra is small, a broad, singly degenerate $A_{1 g}$ band is presumably the only band overlapping the $E_{g}$ band. The strong hybridization between a broad and a narrow band is local in $\vec{k}$ space and the density of states for the ternaries is therefore essentially that of the pure $E_{g}$ band shown in Fig. 7. For a stoichiometric compound like $\mathrm{PbMo}_{6} \mathrm{Se}_{8}$ the number of electrons in the $E_{g}$ band equals the number of holes in the $A_{1 g}$ bands, which is between 0.6 and 2.2. For $\mathrm{PbMo}_{6} \mathrm{~S}_{7.5}$, where on the average one-half $X$ state per cell is missing, there are between 1.6 and 3.2 electrons in the $E_{g}$ band.

There is experimental evidence (see Sec. V) that the Fermi level in $\mathrm{Pb}_{0.92} \mathrm{Mo}_{6} \mathrm{~S}_{7.5}$ lies near a Van Hove singularity and that the Fermi surface has some Mo $s$ character. We now fix the positions of the $A_{1 g}$ bands accordingly. Hence we assume that there are $2.9 E_{g}$ electrons in $\mathrm{Pb}_{0.92} \mathrm{Mo}_{6} \mathrm{~S}_{7.5}$ and, consequently, 3.1 and $2.1 E_{g}$ electrons in, respectively, $\mathrm{PbMo}_{6} \mathrm{~S}_{7.5}$ and $\mathrm{PbMo}_{6} \mathrm{Se}_{8}$. The density of states $N(0)$ per spin and per Mo atom at the respective Fermi levels and for the respective $E_{g}$ bandwidths are given in Table $\mathrm{V}$.

In $\mathrm{Mo}_{6} \mathrm{Se}_{8}$ and $\mathrm{Mo}_{6} \mathrm{~S}_{8}$ the narrow, singly degenerate $A_{u}$ band is raised sufficiently above the position of the $T_{2 u}$ level by the distortion of the octahedra that the $A_{u}$ band overlaps the $E_{g}$ band. The hybridization between the $A_{u}$ and $E_{g}$ bands must be fairly strong and will create additional band broadening and structure in the sum of the density-ofstates curves shown in Fig. 11. For $\mathrm{Mo}_{6} \mathrm{Se}_{8}$ we have given a crude estimate of $N(0)$ in Table $\mathrm{V}$, but for $\mathrm{Mo}_{6} \mathrm{~S}_{8}$, where also the $E_{u}$ band seems to overlap the $E_{g}$ band, even a crude estimate is not possible. The octahedra in $\mathrm{Mo}_{6} \mathrm{Te}_{8}$ are nearly as distorted as in $\mathrm{Mo}_{6} \mathrm{~S}_{8}$ and in $\mathrm{Mo}_{6} \mathrm{Se}_{8}$, but the covalent Mo $d-X p$ mixing is smaller owing to the larger size of the Te atom ( $b^{\prime}$ is larger). This may be seen from the values of $\frac{1}{30}\left|S_{X p}^{\text {Mo } d}\right|^{2} / P_{X p}$ listed in Table IV. In $\mathrm{Mo}_{6} \mathrm{Te}_{6}$ the $E_{g}$ band therefore lies closer to the $T_{2 u}$ level such that it overlaps the $A_{u}$ as well as the $E_{u}$ band and a common fivefold-degenerate band is formed.

\section{Comparison with previous cluster calculations}

Using molecular-orbital LCAO methods, Cotton and Haas, ${ }^{15}$ and later Guggenberger and Sleight, ${ }^{16}$ studied the bonding in cubic (i.e., $b=b^{\prime}$ and $c=0$ ) molybdenum halogen clusters with the formula $\mathrm{Mo}_{6} X_{8}{ }^{4+}$.

Cotton and Haas calculated the pure Mo $d$ states for an isolated octahedron using Slater-type orbitals with varying orbital exponent and they included the mixing with the $X p$ states only to the extent that they excluded the Mo $x y$ orbitals from their basis set. This is not a bad approximation, because, with the inclusion of any reasonable amount of $X p$ intracluster mixing, all the $x y$-like $A_{2 u}, E_{u}$, and $T_{2 g}$ levels will appear above the Fermi level. This may be seen by comparison of Figs. 3 and $9(\mathrm{~d})$. Excluding therefore these levels, our Mo $d$-level scheme in Fig. 3 is qualitatively similar to that of Cotton and Haas for a wide range of orbital exponents and, below the gap, the only difference between the two unhybridized schemes is an inversion of the $T_{2 g}$ and $T_{1 u}$ levels. Our final results including the covalent mixing are, however, significantly different.

Guggenberger and Sleight specifically studied $\mathrm{Mo}_{6} \mathrm{Br}_{8}{ }^{4+}$, which is nearly cubic, and used the extended Hückel molecular-orbital procedure including all the Mo $(5 s, 5 p, 4 d)$ and $\mathrm{Br}(4 s, 4 p)$ atomic orbitals. The Mo $4 d$-orbital exponent was first optimized, to a value consistent with the decay of our muffin-tin orbital, 11 and the Mo valencestate ionization energies, which are analogous to but not equivalent with the negative of our C's were then determined self-consistently with the charge transfer within the $\mathrm{Mo}_{6} \mathrm{Br}_{8}{ }^{4+}$ cluster.

As regards the Mo $d$-like levels below the gap, the results of Guggenberger and Sleight are similar to our cluster results when we neglect the splittings due to trigonal distortion. Contrary to Cotton and Haas, Guggenberger and Sleight found the $T_{2 u}$ level below the $E_{g}$ level and they ascribed this to ligand effects. This is in accord with our description, where, specifically for cubic clusters, we found the $E_{g}-T_{2 u}$ level crossing to take place at $P_{X p}=6$ (estimate for $\mathrm{Mo}_{6} \mathrm{Te}_{8}$ in Sec. IV A).

As regards the Mo $s$-like levels, Guggenberger and Sleight found all six levels well above the Mo $d$ band. This is to be expected from our discussion in Sec. IV A. Moreover, owing to the net positive charge of $\mathrm{Mo}_{6} \mathrm{Br}_{8}{ }^{4+}$, the energies of the $\mathrm{Br} 4 p$ and Mo $5 s$ states, relative to those of the more localized Mo $4 d$ states, are higher than in neutral or slightly negatively charged clusters like the ones we have considered. In particular, we judge that $C_{\mathrm{Mod}}-C_{\mathrm{Br} p} \approx 0.25 \mathrm{Ry}$ and $C_{\mathrm{Mod}}-C_{\mathrm{Mo} s} \approx-0.1 \mathrm{Ry}$ in the Guggenberger-Sleight halogen calculation and, compared with our values in Table III, their potential path lies slightly to the left of ours in Figs. 9 and 10.

Concerning further differences between the 
$\mathrm{Mo}_{6} \mathrm{Br}_{6}{ }^{4+}$ results and our results, it should be noted that our Figs. 9 and 10 apply to noncubic clusters $\left(b^{\prime} / b=0.87-0.92\right)$ and that they include six extra $X$ atoms. These $X$ atoms give rise to the $E_{g}$ and $A_{g}$ levels at the very top of the $X p$ band and, in the Mo $d$ band above the gap, the $3 z^{2}-r^{2}$ derived $T_{1 u}$ and $E_{g}$ levels are pushed upwards, while the $x y$ and $x^{2}-y^{2}$ derived $A_{2 u}, T_{2 g}, E_{u}$, and $A_{2 g}$ levels are unaffected by hybridization from these atoms.

Most recently Mattheiss and Fong ${ }^{17}$ presented a cluster model for the electronic structure of $\mathrm{PbMo}_{6} \mathrm{~S}_{8}$ obtained by means of a tight-binding calculation for a cubic $\mathrm{Mo}_{6} \mathrm{~S}_{8}$ cluster. In this calculation the Mo $5 s$ and $4 d$ and $S 3 s$ and $3 p$ orbitals were included. The interaction parameters, analogous with our C's and two-center integrals (15), were derived in a rather indirect way, namely, from a tight-binding fit to the results of a nonself-consistent augmented-plane-wave (APW) energy-band calculation for a hypothetical $\mathrm{Mo}_{3} \mathrm{~S}$ crystal. In this crystal $\mathrm{Mo}$ is at the face centers and $S$ is at the corners of a fcc lattice with lattice parameter $b$.

The Mattheiss-Fong model for $\mathrm{PbMo}_{6} \mathrm{~S}_{8}$ and our result (Figs. 10 and 11 ) for the properly distorted $\mathrm{Mo}_{6} \mathrm{~S}_{14}$ cluster are similar in general but differ in the important detail that Mattheiss and Fong find the $T_{2 u}$ level slightly above the $E_{g}$ level. These authors therefore predict that, if band broadening and trigonal splittings were included, the Fermi level would lie near the top of a fairly narrow ( $\approx 50 \mathrm{mRy}$ ) complex of five $d x^{2}-y^{2}$ subbands. We now try to understand this discrepancy.

The strong influence of the Mo $d-X p$ covalent mixing on the relative positions of the $T_{2 u}$ and $E_{g}$ levels was demonstrated in Sec. IV A, and was referred to in the discussion of the Cotton-Haas and the Guggenberger-Sleight calculations. First of all, in cubic $\mathrm{Mo}_{6} X_{8}$ clusters, such as those considered by Mattheiss and Fong and (correctly) by Guggenberger and Sleight, we believe that the $X p-$ Mo $d$ two-center integrals are reduced by $\left(b^{\prime} / b\right)^{3}$ $=0.73$ relative to those in $\mathrm{PbMo}_{6} \mathrm{~S}_{7.5}$ and not by $b^{\prime} / b=0.90$ as supposed by Mattheiss and Fong. A consequence of this structure-constant effect is that, in a cubic cluster, the $E_{g}$ level will lie above the $T_{2 u}$ level only if $P_{X p} \lesssim 6$. This seems to be the case for the Guggenberger-Sleight potential path. In the Mattheiss-Fong calculation, there must be a second effect causing their potential path to be shifted in the positive $P_{X p}$ direction. This is consistent with the fact that Guggenberger and Sleight find the $A_{2 g}$ level below the $A_{2 u}$ level [see Fig. $9(d)]$, whereas the reverse is true in the MattheissFong level scheme. A direct comparison of our potential parameters with the two-center para- meters listed in Table I of Mattheiss and Fong, using our Eq. (15) with $b^{\prime} / b=1$, reveals that $C_{\text {Mo } d}$ $-C_{\mathrm{S} p} \approx 0.3 \mathrm{Ry}$ in both calculations and that the Mo $d-$ Mo $d$ interactions are nearly equal, but that the Mattheiss-Fong values for the $\mathrm{S} p-\mathrm{S} p$ and the $\mathrm{S} p-$ Mo $d$ two center integrals are, respectively, five times smaller than and slightly smaller than our values. This is probably connected with the curvature of the potential path: At the center of the $\mathrm{S} p$ levels our value of $\Delta_{\mathrm{S} p}$ is only 0.7 times its value at the center of the Mo $d$ levels but, in a first-principles linear combination of orthogonalized atomic orbitals (LCOAO) calculation, which neglects the three-center integrals, the potential path must be a straight line, whereas, in an LCOAO fit, the curvature is taken approximately into account by using different values of $\Delta$ in the direct and hybridization two-center integrals. In conclusion, we believe that the Mo $d-X p$ covalent mixing is somewhat underestimated in the Mattheiss-Fong model and that this causes an incorrect ordering of the $T_{2 u}$ and $E_{g}$ levels.

Concerning the Mo $s$-like levels, the MattheissFong model, which includes the $s$ orbitals at the eight $X$ sites, corresponds to the dot-dashed potential path in Fig. 10; i.e., all six Mo s-like levels are above the Mo $d$ band.

We originally believed that the turn angle $\phi$, characteristic of the Mo chalcogenides, was determined by the contribution to the total energy from the electrons in the partially filled $E_{g}$ band. This contribution will have a minimum when the $E_{g}$ bandwidth is at its maximum and, as seen in Fig. 8, the correlation between $S_{\text {Mo } d}^{\text {inter }}$ and the observed turn angles is quite good. The $\phi$ dependence of the indirect (Mo $d-X p-$ Mo $d$ ) contribution to $S^{\text {inter }}$ is, however, non-negligible. Moreover, in the following section, VB, we shall estimate the frequency of the $\phi$ torisonal mode and therefore calculate the $\phi$ dependence of the total energy. We find that this is dominated by the sum of the intercluster Mo $d-X p$ interactions over all occupied bands, and our original belief concerning the factor determining the equilibrium value of $\phi$ was therefore an oversimplification. Mattheiss and Fong have come to a similar conclusion, and they furthermore propose that the turn angle $\phi$ is such as to optimize the intercluster Mo $\left(3 z^{2}-r^{2}\right)-X p$ interactions, i.e., that $\phi$ is such as to maximize $n_{\text {Mo-X }}^{\text {inter. }}$

\section{PHYSICAL PROPERTIES}

We shall now discuss our band-structure model in the light of known physical properties of Chevrel-phase compounds and, as a first step, we shall try to use experimental evidence to eliminate the 
uncertainty in the position of the Mo $s$-like bands obtained with our model. The fact ${ }^{18}$ that the pressure dependence of the superconducting transition temperature is abnormally large in $\mathrm{Pb}_{0.92} \mathrm{Mo}_{6} \mathrm{~S}_{7.5}$ leads us to place the Fermi level in this compound at a pronounced Van Hove singularity. Using Fig. 11 , we therefore choose the singularity in the upper half of the $E_{g}$ band and hence assume that the number of $E_{g}$ electrons is 2.9 in $\mathrm{Pb}_{0.92} \mathrm{Mo}_{6} \mathrm{~S}_{7.5}$ (Fig. 7) and, consequently, $2.1+2(m-1+x)$ in $M_{m} \mathrm{Mo}_{6} X_{8-x^{\circ}}$. This leaves 0.1 holes in the $A_{1 g}^{\prime}$ band and thus places the potential path near the dotdashed curve in Fig. 10. NMR studies ${ }^{19}$ in $\mathrm{Eu}_{0.5} \mathrm{Sn}_{0.5} \mathrm{Mo}_{6} \mathrm{~S}_{8}$ have shown a (negative) $s$ polarization at the Mo site and hence indicate some Mo $s$ character at the Fermi surface. This is consistent with the presence of an $A_{1 g}^{\prime}$-hole sheet. [It would, however, have been even more consistent with the presence of an $A_{18}$-electron sheet which, according to Fig. 10, would have a much higher Mo-s probability. We can therefore not entirely rule out the possibility that the potential path lies near the full curve in Fig. 10, that the Fermi level in $\mathrm{Pb}_{0.92} \mathrm{Mo}_{6} \mathrm{~S}_{7.5}$ lies at the Van Hove singularity in the lower half of the $E_{g}$ band, and that the number of $E_{g}$ electrons in $M_{m} \mathrm{Mo}_{6} X_{8-x}$ is $0.3+2(m-1+x)$.]

\section{A. Paramagnetic susceptibility and electronic-specific-heat coefficient}

The paramagnetic susceptibilities of $\mathrm{Mo}_{6} \mathrm{~S}_{8}$, $\mathrm{PbMo}_{5.1} \mathrm{~S}_{6}$, and $\mathrm{SnMo}_{5} \mathrm{~S}_{6}$, as measured by Chevrel et al.,${ }^{3}$ Bader et al.,${ }^{20}$ and Morton et al.,${ }^{21}$ yield $N_{\mathrm{x}}(0)=15,21$, and 19 states/(spin Mo-atom Ry) for the density of states at the Fermi level when the expression for the unenhanced Pauli spin susceptibility is used. For the above-mentioned $\mathrm{Pb}$ and Sn compounds the experimental electronicspecific-heat coefficients ${ }^{20}$ yield $N_{\gamma}(0)=40$ and 35 states/(spin Mo-atom Ry) when the effect of electron-phonon enhancement is neglected.

From self-consistent, spin-polarized bandstructure calculations, ${ }^{22}$ employing the local-spindensity approximation for exchange and correlation, we have found the value $I_{x c}=40 \mathrm{mRy}$ for the effective exchange-interaction parameter in Mo metal. Since this interaction is essentially intraatomic, its value must be nearly the same in the Mo chalcogenides and, for the bare band density of states in $\mathrm{Mo}_{6} \mathrm{~S}_{8}, \mathrm{PbMo}_{5.1} \mathrm{~S}_{6}$, and $\mathrm{SnMo}_{5} \mathrm{~S}_{6}$, we therefore derive $N(0)=\left[N_{\chi}(0)^{-1}+I_{x c}\right]^{-1}=9.4,11.4$, and 10.8 states/(spin Mo-atom Ry), assuming that the measured susceptibilities arise from spin only. The susceptibility enhancements are therefore around 1.7 and the electron-phonon enhancements in the ternaries are $1+\lambda \approx 3.5$.

In $\mathrm{Mo}_{6} \mathrm{~S}_{8}$ the substantial overlap between the
$E_{g}, A_{u}$, and $E_{u}$ subbands prevented us from estimating $N(0)$ theoretically, but the above-mentioned value of 9.4 has the order of magnitude to be expected from Fig. 11. In the nonstoichmetric compounds $\mathrm{PbMo}_{5.1} \mathrm{~S}_{6}$ and $\mathrm{SnMo}_{5} \mathrm{~S}_{6}$ the $\mathrm{Mo}_{6}$ octahedra are probably not intact and it is therefore difficult to make comparisons with our model for a quantity as sensitive as $N(0)$. The above-mentioned values of 11.4 and 10.8 nevertheless equal our theoretical value of 11 for $\mathrm{PbMo}_{6} \mathrm{~S}_{7.5}$.

$$
\text { B. Torsional mode of } \mathrm{Mo}_{6} \mathrm{X}_{8} \text { units }
$$

Heat-capacity and inelastic-neutron-scattering studies ${ }^{5}$ of the phonon spectra of $\mathrm{Mo}_{6} \mathrm{Se}_{8}, \mathrm{PbMo}_{5.1} \mathrm{~S}_{6}$, and $\mathrm{SnMo}_{5} \mathrm{~S}_{6}$ are claimed to indicate that the $\mathrm{Mo}_{6} X_{8}$ clusters can be regarded as quasirigid units for which the frequencies of the three torsional modes are about $12 \mathrm{meV} \approx 140 \mathrm{~K}$.

We shall estimate the frequency of the $\phi$ mode (see Fig. 1) at zero wave vector by calculating the $\phi$ dependence of the sum of the one-electron energies for fixed potential parameters $C_{t l}$ and $\Delta_{t i}$. The hard-core repulsion, which in a self-consistent calculation ${ }^{22}$ employing a local approximation for exchange and correlation ${ }^{9}$ would mainly enter through $\phi$-dependent band positions $C_{t l}$, will be treated empirically. At equilibrium the interclus-

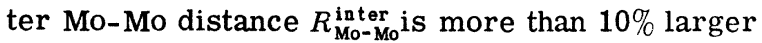
than the intracluster (and the metallic) Mo-Mo distance, whereas the intercluster Mo- $X$ distance $R_{\text {Mo-X }}^{\text {inter }}$ is only a few percent larger than the intracluster Mo- $X$ distance (see Table I). Moreover, the $\phi$ dependence of $R_{\text {Mo- } X}^{\text {inter }}$ is much stronger than that of $R_{\mathrm{Mo}-\mathrm{Mo}}^{\mathrm{inter}}$. We shall therefore represent the repulsion by the Lennard-Jones form $A\left(s / R_{\mathrm{Mo}-X}^{\mathrm{inter}}\right)^{12}$, where $A$ is an adjustable parameter.

Upon increasing $\phi$ and hence decreasing $R_{\mathrm{Mo}-X}^{\mathrm{inter}}$ the $X p$ band energies will fall owing to the increased mixing with the Mo $d$ band and, summing over the entire $X p$ band by means of (21) and (22), we obtain the attractive term $-B_{X p}\left(s / R_{\mathrm{Mo}-X}^{\text {inter }}\right)^{8}$, where

$$
\begin{aligned}
B_{X p} & =-2 \Delta_{X p} P_{\text {Mo } d}\left(C_{X p}\right)^{-1} \times 900 \times 6 \\
& =2 \Delta_{X p} \Delta_{\text {Mo } d}\left(C_{\text {Mo } d}-C_{X p}\right)^{-1} \times 5400 .
\end{aligned}
$$

The factor of 2 arises from the spin degeneracy, the potential parameters refer to the center of the $X p$ band, and the factor of 6 arises because each of the six $X$ atoms at general positions carries one intercluster Mo- $X$ bond. This attraction is counterbalanced by the corresponding increase of the Mo $d$ energies to the extent that these are occupied. For each of the 9 or 10.5 occupied Mo $d$ subbands we therefore compute the shift given by (17a). The sum of these terms, however, differs insignificantly from the result $B_{\text {Mo } d}\left(s / R_{\text {Mo-X }}^{\text {inter }}\right)^{8}$, obtained 
by using (22) instead of (18a). The $\phi$ dependences not included in $R_{\mathrm{Mo}-X}^{\mathrm{inter}}$ consequently even up. The constant $B_{\text {Mo } d}$ equals $9 / 30$ or $10.5 / 30$ times the formal expression for $B_{X p}$ with potential parameters referring to the occupied part of the Mo $d$ band. The resulting attraction arising from the Mo $d-X p$ intercluster hybridization is therefore $-B\left(s / R_{\mathrm{Mo}-X}^{\text {inter }}\right)^{8}$, and we find that $B=B_{X p}-B_{M o d} \approx 40$ Ry for both $\mathrm{Mo}_{6} \mathrm{Se}_{8}$ and $\mathrm{PbMo}_{6} \mathrm{~S}_{7.5}$.

Also the Mo $d$-Mo $d$ interactions contribute to the intercluster attraction through broadening of the Mo $d$ band; more specifically, through broadening of, and repulsion between, the Mo $d$ subbands as discussed at the beginning of Sec. III. Taking again the average over all subbands, the attractive contribution is seen to be

$$
\begin{aligned}
&-2 n_{\text {Mo } d}\left(1-\frac{1}{30} n_{\text {Mo } d}\right) \frac{1}{4}\left(\partial W^{2} / W^{2}\right) W \Delta_{\text {Mo } d} \\
& \approx-0.4 \mathrm{Ry}\left(R_{\text {Mo-Mo }}^{\text {intra }} / R_{\text {Mo-Mo }}^{\text {inter }}\right)^{10},
\end{aligned}
$$

where $n_{\text {Mo } d}$ is the number of occupied Mo $d$ bands, i.e., 9 or 10.5. For the equilibrium distances given in Table I, the Mo $d-X p$ intercluster attraction considered above is $-0.5 \mathrm{Ry}$, while the Mo $d-$ Mo $d$ attraction considered here is only $-0.06 \mathrm{Ry}$, and it may therefore be neglected. Also interactions involving Mo $s$ states may be neglected.

With the total energy in the form

$$
U=A\left(s / R_{\mathrm{Mo}-X}^{\text {inter }}\right)^{12}-B\left(s / R_{\mathrm{Mo}-X}^{\text {inter }}\right)^{8},
$$

we now adjust $A$ such that the minimum occurs at the observed $R_{\mathrm{Mo}-x}^{\mathrm{inter}}$ and then find the force constant $d^{2} U / d \phi^{2}=10 \mathrm{Ry}$ for both compounds. Finally, a calculation of the moments of inertia $J$ about the threefold axis for the $\mathrm{Mo}_{6} \mathrm{Se}_{8}$ and $\mathrm{Mo}_{6} \mathrm{~S}_{8}$ units yields the respective frequencies

$$
h \nu=\left[\left(d^{2} U / d \phi^{2}\right) J^{-1}\right]^{1 / 2}=10 \text { and } 15 \mathrm{meV} .
$$

This is in excellent agreement with the experiments.

It may be noted that changing the power in the repulsive term between 10 and 15 will change the frequencies by only $\pm 25 \%$. Moreover, we have neglected interactions with the $\mathrm{Pb}$ and $\mathrm{Sn}$ atoms.

\section{Average electron-phonon matrix element}

The experimental superconducting transition temperatures for the compounds listed in Table $\mathrm{V}$ are $T_{c}=1.6,6.3,13,3.9$, and $0 \mathrm{~K}$, respectively. One usually assumes that $T_{c}$ depends primarily on the magnitude of the electron-phonon mass enhancement, which in McMillan's approximation ${ }^{23}$ is $\lambda=N(0)\left\langle I^{2}\right\rangle / M\left\langle\omega^{2}\right\rangle$. Here, $\left\langle I^{2}\right\rangle$ is the square of the electron-phonon matrix element averaged over the Fermi surface, $M$ is the atomic mass, and $\left\langle\omega^{2}\right\rangle$ is an appropriate average of the squared phonon frequency.
For the Mo chalcogenides of high $T_{c}$ we have seen that $N(0)$ essentially derives from the Mo $d$ like $E_{g}$ band and that it depends sensitively on the geometry, through the intercluster coupling constant (Table V), and on the stoichiometry, through the position of the Fermi level within the $E_{g}$-band density-of-states curve shown in Fig. 7. For $\mathrm{PbMo}_{6} \mathrm{~S}_{7.5}$ we estimated that $N(0)=11$ states $/$ (spin Mo-atom Ry) and this may be compared with the value of 4 for bcc Mo metal ${ }^{24,25}$ where $T_{c}=0.9 \mathrm{~K}$ and $\lambda=0.4$. We shall now estimate $\left\langle I^{2}\right\rangle$.

Using the Gaspari-Gyorffy approximation ${ }^{26}$ with rigid atomic spheres, it may be $\operatorname{shown}^{24}$ that

$$
\begin{array}{rl}
s_{t}^{2} N^{2}\left\langle I_{t}^{2}\right\rangle=\sum_{l} & 2 \tilde{l}(2 \tilde{l}+1)^{-1}(2 l+1)^{-1}\left(\partial n / \partial D_{t l}\right)\left(\partial n / \partial D_{t}\right) \\
& \times\left[\left(D_{t l}-l\right)\left(D_{t} \tilde{l}+\tilde{l}+1\right)+\left(E_{F}-v_{t}\right) s_{t}^{2}\right]^{2}
\end{array}
$$

where $N \equiv N(0) \equiv N\left(E_{F}\right)$ and we have considered the contribution from the transition atoms only ( $t=\mathrm{Mo})$. Moreover, $\tilde{l} \equiv l+1$, the value of the one-electron potential at the transition-atom sphere is $v_{t}\left(s_{t}\right)$ $\equiv v_{t}$, and the value of the logarithmic derivative function [see (2)] at the Fermi energy is $D_{t l}\left(E_{F}\right)$ $\equiv D_{t l}$. The derivatives of the canonical number of states function [see (7a) and (9)] with respect to the logarithmic derivative may be related to the projected density of states and the normalized partial wave, evaluated at the sphere, through

$$
\partial n / \partial D_{t l}=N_{t l}\left(E_{F}\right) / \dot{D}_{t l}\left(E_{F}\right)=-s_{t} \phi_{t l}^{2}\left(E_{F}, s_{t}\right) N_{t z}\left(E_{F}\right) \text {. }
$$

However, we prefer the canonical formulation in which the atomic and structural dependences are separated, and therefore write

$$
\begin{aligned}
\partial n / \partial D_{t l} & =\left(d P_{t l} / d D_{t l}\right) n_{t l}^{\prime}(\overrightarrow{\mathrm{P}}) \\
& =-2(2 l+1)^{2}\left(s / s_{t}\right)^{2 l+1}\left(D_{t l}-l\right)^{-2} n_{t l}^{\prime} \\
& =-\frac{1}{2}\left(s_{t} / s\right)^{2 l+1}\left(D_{t l}+l+1\right)^{-2} P_{t l}^{2} n_{t l}^{\prime} .
\end{aligned}
$$

For transition atoms only the $p d$ and the $d f$ terms contribute significantly to (32) because the expression in the square brackets vanishes if both the $l$ and the $\tilde{l}$ electrons at the Fermi energy are free-electron-like with respect to $v_{t}$ [Eq. (2.30) in Ref. 6] and because the $d$ projected density of states usually dominates the total density of states. Retaining the $p d$ and $d f$ contributions only, using (33), and dropping the subscripts $t$, the electronphonon matrix element may be written

$$
\begin{aligned}
\left\langle I^{2}\right\rangle \approx & \frac{\left(N_{d} / N\right)^{2}}{\mu_{d}^{2} s_{t}^{6}} \\
\times & \left\{\frac{20}{3}\left(\frac{s_{t}}{s}\right)^{8} \frac{P_{p}^{2} n_{p}^{\prime}}{n_{d}^{\prime}}\left[\frac{\left(D_{p}-1\right)\left(D_{d}+3\right)+\left(E_{F}-v\right) s_{t}^{2}}{\left(D_{p}+2\right)\left(D_{d}-2\right)}\right]^{2}\right. \\
& \left.+\frac{30}{7}\left(\frac{s_{t}}{s}\right)^{12} \frac{P_{f}^{2} n_{f}^{\prime}}{n_{d}^{\prime}}\left[1+\frac{\left(E_{F}-v\right) s_{t}^{2}}{\left(D_{f}+4\right)\left(D_{d}-2\right)}\right]^{2}\right\} .
\end{aligned}
$$


If, at the Fermi level, we use the approximation of weak hybridization (Sec. II D), which, loosely speaking, amounts to describing the Mo $p$ and Mo $f$ waves by the tails of the Mo $d$ orbitals, we realize from (19b) and (19c) that the structural quantities $P_{l^{\prime}}^{2} n_{l^{\prime}}^{\prime} / n_{d}^{\prime}$ with $l^{\prime}=1$ or 3 , are the average of the hybridization structure constants over the Fermi surface, i.e.,

$$
\begin{aligned}
P_{l^{\prime}}^{2} n_{l^{\prime}}^{\prime} / n_{d}^{\prime} \\
\quad=\langle| S_{\left.\left.\mathrm{Mo}_{l^{\prime}}(\overrightarrow{\mathrm{k}})\right|_{j} ^{2}\right\rangle}^{\mathrm{M}} \\
\quad=\frac{\sum_{j} V_{\mathrm{BZ}}^{-1} \int d^{3} k \delta\left(f_{j}\left(\overrightarrow{\mathrm{k}}, \overrightarrow{\mathrm{P}}\left(E_{F}\right)\right)\right)\left|S_{\mathrm{Mo} l^{\prime}}^{\mathrm{Mo}}(\overrightarrow{\mathrm{k}})\right|_{j}^{2}}{\sum_{j} V_{\mathrm{BZ}}^{-1} \int d^{3} k \delta\left(f_{j}\left(\overrightarrow{\mathrm{k}}, \overrightarrow{\mathrm{P}}\left(E_{F}\right)\right)\right)} .
\end{aligned}
$$

These are the quantities which make $\left\langle I^{2}\right\rangle$ for the Chevrel-phase differ significantly from that of Mo metal.

Let us first consider the average of the hybridization structure constants over the entire $d$ band. From (22),

$$
\left.\begin{array}{l}
\frac{1}{30}\left|S_{\mathrm{Mo} p}^{\mathrm{Mod}}\right|^{2} \\
\frac{1}{5}\left|S_{\text {Mo } p}^{\mathrm{Mod}}\right|^{2}
\end{array}\right\}=180 \sum_{\overrightarrow{\mathrm{R}} \neq 0}^{\infty}\left(\frac{s}{R}\right)^{8}=\left\{\begin{array}{c}
6.7 \mathrm{Chevrel} \\
20 \mathrm{fcc} \\
20 \mathrm{bcc}
\end{array},\right.
$$

where, in the fcc and bcc structures with one atom per primitive cell, there are 5 rather than $30 d$ bands. Moreover,

$$
\left.\begin{array}{c}
\frac{1}{30}\left|S_{\text {Mo } f}^{\text {Mo } d}\right|^{2} \\
\frac{1}{5}\left|S_{\text {Mo } f}^{\text {Mo }}\right|^{2}
\end{array}\right\}=5880 \sum_{\overrightarrow{\mathrm{R}} \neq 0}^{\infty}\left(\frac{s}{R}\right)^{12}=\left\{\begin{array}{l}
20 \text { Chevrel } \\
58 \mathrm{fcc} \\
61 \mathrm{bcc} .
\end{array}\right.
$$

Hence, the constants are nearly identical in the closely packed structures, provided that $s$ is the Wigner-Seitz radius, but the constants in the Chevrel phase are about three times smaller than in the fcc phase because the Chevrel phase has 4 rather than 12 nearest neighbors.

For the Fermi-surface averages in bcc Mo, it has been found ${ }^{24,25}$ that $P_{p}^{2} n_{p}^{\prime} / n_{d}^{\prime} \approx 20$ and $P_{f}^{2} n_{f}^{\prime} / n_{d}^{\prime}$ $\approx 120$, which are, respectively, the same as and twice as large as the averages given above. In the Chevrel phase we shall now use (35) and should therefore calculate $\left|S_{\mathrm{Mo} l^{\prime}}^{\mathrm{Mod}}(\overrightarrow{\mathrm{k}})\right|_{E_{g}}^{2}$ for the two $E_{g}$ bands from (18b). When expanding the $d$ orbitals from the neighbors in $p$ and $f$ waves about a given site it is reasonable to neglect all but the nearestneighbor orbitals and hence to retain the intracluster contributions only. With this approximation, the average of $\left|S_{M_{0} l^{\prime}}^{\mathrm{Mod}}(\overrightarrow{\mathrm{k}})\right|_{E_{g}}^{2}$ over the three $\overrightarrow{\mathrm{k}}$ points on the Fermi surface which are related by the threefold axis [i.e., those obtained from a given $\overrightarrow{\mathrm{k}}=\left(k_{i}, k_{j}, k_{k}\right)$ by cyclically permuting $k_{i}, k_{j}$, and $k_{k}$ ] is independent of $\overrightarrow{\mathrm{k}}$ and the band index, and equals $\left|S_{\text {Mo } l^{\prime}}^{\text {Mod }}\right|_{E_{g}}^{2}$ as given by (18a) for an $E_{g}$ state of the octahedron (Fig. 4). We therefore obtain

$$
\begin{aligned}
\left\langle\left|S_{\text {Mo } p}^{\text {Mo } d}(\overrightarrow{\mathrm{k}})\right|_{E_{g}}^{2}\right\rangle & \\
= & \left|S_{\text {Mo }_{p}}^{\text {Mo } d}\right|_{E_{g}}^{2}=\frac{6}{6}\left[\mid 2 S\left(z, y^{2}-z^{2} ; \frac{1}{2} b, 0, \frac{1}{2} b\right) \exp \left(-\frac{2}{3} \pi i\right)\right. \\
& +2 S\left(z, z^{2}-x^{2} ; 0, \frac{1}{2} b, \frac{1}{2} b\right) \exp \left(\left.\frac{2}{3} \pi i\right|^{2}\right. \\
& \left.+|2 S(y, \ldots) \ldots|^{2}+|2 S(z, \ldots) \ldots|^{2}\right] \\
= & \left|2 S\left(z, y^{2}-z^{2} ; \frac{1}{2} b, 0, \frac{1}{2} b\right)(-i \sqrt{3})\right|^{2}+0+0 \\
= & \left(12 \times \frac{180}{32}\right)\left(s / R_{\text {Mo-Mo }}^{\text {intra }}\right)^{8}=0.6 .
\end{aligned}
$$

This Fermi-surface average only amounts to $\frac{3}{32}$ of the average (36) over the entire $d$ band, and the neglect of the intercluster contributions may therefore lead to a large relative error in the $p d$ term which, however, is insignificant compared with the $d f$ term. For the $d f$ contribution,

$$
\begin{aligned}
\left\langle\left|S_{\text {Mo } f}^{\text {Mo } d}(\overrightarrow{\mathrm{k}})\right|_{E_{g}}^{2}\right\rangle= & \left|S_{\text {Mo } f}^{\text {Mo } f}\right|_{E_{g}}^{2} \\
= & \mid 2 S\left(\left(5 z^{2}-3 r^{2}\right) z, y^{2}-z^{2} ; \frac{1}{2} b, 0, \frac{1}{2} b\right) \\
& \times\left.\left[\exp \left(-\frac{2}{3} \pi i\right)-\exp \left(\frac{2}{3} \pi i\right)\right]\right|^{2} \\
& +\mid 2 S\left(\left(x^{2}-y^{2}\right) z, y^{2}-z^{2} ; \frac{1}{2} b, 0, \frac{1}{2} b\right) \\
& \times\left.\left[\exp \left(-\frac{2}{3} \pi i\right)+\exp \left(\frac{2}{3} \pi i\right)\right]\right|^{2} \\
= & 16264\left(s / R_{\text {Mo-Mo }}^{\text {intra }}\right)^{12}=13 .
\end{aligned}
$$

This amounts to 0.7 times the average (37) over the entire $d$ band.

As a result, the values of the structural quantities $P_{p}^{2} n_{p}^{\prime} / n_{d}^{\prime}$ and $P_{f}^{2} n_{f}^{\prime} / n_{d}^{\prime}$ are smaller in the Chevrel phase than in the bcc phase by factors of 30 and 9 , respectively.

Returning now to the remaining quantities in (34), the ratio of the $d$ to the total density of states, $N_{d} / N$, takes the value 0.79 in bcc $\mathrm{Mo}^{24,25}$ and 0.85 in the Chevrel phase (Sec. IV A). The values of $\mu_{d}$ and $s_{t}$ (Table III) are the same in the Chevrel and the bcc phases, and so are the values of the quantities entering the square brackets of (34). Specifically: From Fig. 11, Table III, and Eq. (2) we find $D_{d}=-3.5$ and $D_{p}=0.2$. Moreover, $\left(E_{F}-v\right) s_{t}^{2}$ $=7.4$. [This is twice the value $\left(\frac{9}{4} \pi\right)^{2 / 3}$ which is appropriate for the Fermi energy corresponding to one free $s p$ electron per Mo atom and which nearly equals $\left(E_{F}-V_{s}\right) s_{t}^{2} \approx\left(E_{F}-V_{p}\right) s_{t}^{2}$, the $V^{\prime} s$ being the square-well pseudopotentials defined in (12). As pointed out by Pettifor, ${ }^{24}$ it is the large effective core of the Mo ion which pushes the $s p$ pseudopotential far above the value $v$ of the oneelectron potential at the sphere and thereby enhances the (small) $p d$ contribution to $\left\langle I^{2}\right\rangle$.] As a result,

$$
\frac{\left[\left(D_{p}-1\right)\left(D_{d}+3\right)+\left(E_{F}-v\right) s_{t}^{2}\right]^{2}}{\left[\left(D_{p}+2\right)\left(D_{d}-2\right)\right]^{2}}=0.40 .
$$

The $f$-potential parameters are $\left(V_{f}-v\right) s_{t}^{2}=-0.9$, $\tau_{f}=1$, and $\gamma_{f}=\frac{11}{252}$ (this is the free-electron value ${ }^{6}$ ) and the logarithmic derivative at the Fermi level 
is therefore $D_{f}=2.0$. As a result,

$$
\left[1+\left(E_{F}-v\right) s_{t}^{2} /\left(D_{f}+4\right)\left(D_{d}-2\right)\right]^{2}=0.60 .
$$

We can finally evaluate (34). For bcc Mo metal

$$
\begin{aligned}
\left\langle I^{2}\right\rangle & =0.79^{2} /\left(4.0 \times 2.92^{3}\right)^{2} \\
& \times\left(\frac{20}{3} \times 20 \times 0.40+\frac{30}{7} \times 120 \times 0.60\right) \\
& =6.3 \times 10^{-5}(53+310) \\
& =23 \times 10^{-3}(\mathrm{Ry} / \text { Bohr radius })^{2},
\end{aligned}
$$

which equals the value of Butler. ${ }^{25}$ For $\mathrm{PbMo}_{6} \mathrm{~S}_{7.5}$

$$
\begin{aligned}
\left\langle I^{2}\right\rangle= & 0.85^{2} /\left(4.0 \times 2.92^{3}\right)^{2} \\
& \times\left(\frac{20}{3} \times 1.03^{8} \times 0.6 \times 0.40+\frac{30}{7} \times 1.03^{12} \times 13 \times 0.60\right) \\
& =7.3 \times 10^{-5}(2+48) \\
& =3.6 \times 10^{-3}(\mathrm{Ry} / \text { Bohr radius })^{2},
\end{aligned}
$$

and for $\mathrm{Mo}_{6} \mathrm{Se}_{8}$ and $\mathrm{PbMo}_{6} \mathrm{Se}_{8}\left\langle I^{2}\right\rangle=2.9 \times 10^{-3}$ and $3.5 \times 10^{-3}$ (Ry/Bohr radius) ${ }^{2}$. For the Mo chalcogenides the differences in $\left\langle I^{2}\right\rangle$ are due to the factor $\left(s_{t} / s\right)^{12} \propto b^{-12}$, which is a measure for how closely packed the Mo octahedron is in the various compounds.

For the electronic contribution $\eta \equiv N(0)\left\langle I^{2}\right\rangle$ to the electron-phonon mass enhancement we find, using the values of $N(0)$ given in Table $\mathrm{V}, \eta=1.0,1.9$, and $1.4 \mathrm{eV} / \AA^{2}$ in $\mathrm{Mo}_{6} \mathrm{Se}_{8}, \mathrm{PbMo}_{6} \mathrm{~S}_{7.5}$, and $\mathrm{PbMo}_{6} \mathrm{Se}_{8}$, respectively. For the two latter materials, in which we believe that there is no overlap with the $A_{u}$ band at the Fermi level, these $\eta$ values correlate reasonably with the superconducting transition temperatures.

A somewhat surprising result of Sec. VA was that the electron-phonon enhancement $\lambda$ in the typical, high- $T_{c}$ Mo chalcogenide $\mathrm{PbMo}_{6} \mathrm{~S}_{7.5}$ seemed to be as large as 2.5. By contrast, for bcc Mo metal, ${ }^{25} T_{c} \approx 0.9 \mathrm{~K}$ and $\lambda \approx 0.40$. With $N(0)=4.0$ states/(spin Mo-atom Ry) we find $\eta=4.5 \mathrm{eV} / \AA^{2}$ in bcc Mo. Using these values of $\lambda$ and $\eta$ in McMillan's expression, the ratio between the average phonon frequencies should be

$$
\left(\left\langle\omega^{2}\right\rangle_{\text {chalc }} /\left\langle\omega^{2}\right\rangle_{\text {metal }}\right)^{1 / 2}=[(0.4 / 2.5)(1.9 / 4.5)]^{1 / 2}=0.26 \text {. }
$$

Since the effective Debye temperatures, or rather their high-temperature limits, are about 500 and $400 \mathrm{~K}$ in the Mo chalcogenide ${ }^{5}$ and in Mo metal, ${ }^{25}$ the above-mentioned ratio is only reasonable if low-frequency modes, like the rocking mode with $h \nu \approx 140 \mathrm{~K}$ considered in Sec. V B, are of particular importance for the electron-phonon interaction in the Mo chalcogenides.

More realistic calculations, avoiding the approximations of McMillan and of Gaspari and Gyorffy should soon be possible. ${ }^{27-29}$

\section{Critical fields}

An understanding of the very high critical magnetic fields may be obtained from our band structure. Pair breaking in a strong magnetic field should limit superconductivity within the Clogstonlimit: $H_{p 0}=\Delta_{0}\left(\sqrt{2} \mu_{B}\right)^{-1}=18.4 T_{c} \mathrm{kG} / \mathrm{K}$, which is only $240 \mathrm{kG}$ for $T_{c}=13 \mathrm{~K}$. Here $\mu_{B}$ is the Bohr magneton and $\Delta_{0}$ the gap parameter at $0 \mathrm{~K}$ and zero magnetic field. Fischer ${ }^{2}$ suggested that strong spin-orbit coupling would change the pairing conditions and the Clogston limit could not be valid. Some evidence to support this can be found from estimating $\tau_{\text {so }}$ from a band-structure point of view.

The $E_{g}$ band is shown in the upper panel of Fig. 6 , and in Fig. 12 we have sketched the $E_{g}$ sheet of the Fermi surface estimated in $\mathrm{PbMo}_{6} \mathrm{~S}_{7.5}$. In the first-order approximation, where the Bloch states in the $E_{g}$ band have $x^{2}-y^{2}$ character exclusively, i.e., where there are only $|m|=2$ partial-wave components at each Mo-site, the spin-orbit coupling and the trigonal distortion are both ineffective, so that the electron and hole sheets touch at all the body diagonals of the Brillouin zone. The interband mixing responsible for the subband repulsion found in Sec. III and that arising from the $X p$ covalent mixing and the distortion of the octahedra will, however, reduce the symmetry of the $E_{\mathrm{g}}$ band from

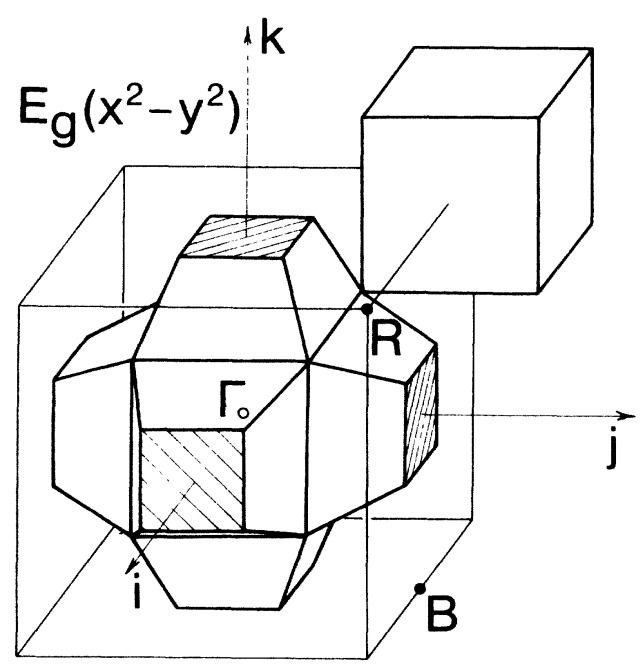

FIG. 12. Sketch of the $E_{g}$ constant-energy surface which holds $1.1 E_{g}$ electrons per cell (see Fig. 7). The hole surface is open along the $\langle 100\rangle$ directions and the electron surface is closed. The surface which holds $2.9 E_{E}$ electrons and which we believe forms the heavy part of the Fermi surface in $\mathrm{Pb}_{0.92} \mathrm{Mo}_{6} \mathrm{~S}_{7.5}$ is topologically equivalent with the surface shown. The open surface will, however, be centered at $R$ and will be an electron surface, while the closed surface will be centered at $\Gamma$ and will be a hole surface. 
cubic to trigonal, and they will produce gaps at all other than the [111] diagonal. They will, furthermore, introduce $d$ components with $|m|=1$ whereby the spin-orbit coupling becomes effective in the small regions near the remaining [111] degeneracy. Being a second-order effect, the resulting splitting $E_{\mathrm{so}}$ is a sensitive function of the local environment in nonperfect crystals and its value is only a fraction of the spin-orbit parameter $\xi_{\text {Mod }}$ $=8 \mathrm{mRy}$. For $\mathrm{PbMo}_{6} \mathrm{~S}_{7.5}$ we find $E_{\mathrm{so}}=1.5 \mathrm{mRy}$ in the central part of the band. It may be noted that the overlap of the $A_{1 g}$ band alone has no effect on the spin-orbit splitting, because the $d$ components from that band have $m=0$.

Introducing the definition $\tau_{\mathrm{so}} \equiv \frac{1}{2} \hbar E_{\mathrm{so}}^{-1}$ into the the ory of $\mathrm{Maki}^{30}$ for $\mathrm{H}_{c 2}$, we obtain the coupling constant $\lambda_{\text {so }}=(4 / 3 \pi)\left(E_{\mathrm{so}} / k_{B} T_{c}\right)$. For $\mathrm{Pb}_{0.94} \mathrm{Mo}_{6} \mathrm{~S}_{7.5}$ and $\mathrm{Sn}_{1.13} \mathrm{Mo}_{6} \mathrm{~S}_{7.5}$, which have $T_{c}=13$ and $12 \mathrm{~K}$, respectively, ${ }^{31}$ we find $\lambda_{\mathrm{so}}=8$ in both cases and this exceeds the usual values considerably. Using the experimental ${ }^{31}$ slopes $\left(-d H_{c 2} / d T\right)_{T_{c}}$, we find the Maki parameters $\alpha=3.25$ and 2.40, and with our values of $\lambda_{\text {so }}$ we then obtain the critical fields $H_{c 2}(0)$ $=420$ and $320 \mathrm{kG}$ for $\mathrm{Pb}_{0.94} \mathrm{Mo}_{6} \mathrm{~S}_{7.5}$ and $\mathrm{Sn}_{1.13} \mathrm{Mo}_{6} \mathrm{~S}_{7.5}$, respectively. The experimental values of $\alpha$ are consistent with our density-of-states curve in Fig. 7 if, as is usually assumed, $\alpha$ is proportional to the density of states squared and if there are about 2.9 and $3.4 E_{\mathrm{g}}$ electrons in $\mathrm{Pb}_{0.94} \mathrm{Mo}_{6} \mathrm{~S}_{7.5}$ and $\mathrm{Sn}_{1.13} \mathrm{Mo}_{6} \mathrm{~S}_{7.5}$, respectively.

We have to remember that Maki's theory applies to weak-coupling superconductors, whereas the specific-heat measurements ${ }^{20}$ mentioned in Sec. $\mathrm{V} A$ indicate a strong-coupling behavior of the Chevrel phases. Rainer and Bergmann ${ }^{32}$ have solved the problem of connecting weak- and strongcoupling theories by calculating an enhancement factor $\eta(T)$ such that

$$
H_{c 2}(T)=\eta(T) H_{c 2}{ }^{\text {weak coupling }}(T) .
$$

This enhancement factor can be estimated from the ratio $T_{c} /\langle\omega\rangle$, where $\langle\omega\rangle$ is an average phonon frequency. If we choose $\mathrm{e}^{5,20}\langle\omega\rangle=\omega_{D}=74 \mathrm{~K}$ for both compounds, we find $\eta(0) \simeq 1.26$, which finally yields $H_{c 2}=530$ and $400 \mathrm{kG}$, in good correspondence with the experimental values ${ }^{31}$ of 540 and $325 \mathrm{kG}$.

In single crystals we would, however, expect strong anisotropy effects because the spin-orbit splitting is effective only in the [111] direction. On purely geometrical reasoning, one could expect a maximum factor of 3 , and this seems to have been observed. ${ }^{33}$

We finally would like to point out that, owing to the strong admixture of $|m|=1$ components in the $T_{2 u}\left(x^{2}-y^{2}\right)$ states (Fig. 5), the spin-orbit splitting in the $T_{2 u}$ bands is of order $10 \mathrm{mRy}$, and the critical fields estimated as above would exceed the ex- perimentally observed fields by more than $100 \mathrm{kG}$. On the other hand, for these bands the spin-orbit coupling is not a sensitive second-order effect and the identification of $\tau_{\mathrm{so}}$ with $E_{\mathrm{so}}$ is not justified. We believe that this confirms our conclusion that the $E_{g}$ band rather than the $T_{2 u}$ band, or an admixture of these five bands, is the conduction band in the high-critical-field Mo chalcongenides.

\section{CONCLUSION}

We have investigated the band structures of the Chevrel-phase molybdenum chalcogenides $M_{m} \mathrm{Mo}_{6} X_{8-x}$, with $M$ being a large, divalent cation, using as a model the atomic-sphere approximation to the KKR and LMTO methods. The canonical properties of this model have allowed us to obtain simple analytical estimates as well as numerical solutions for a continuous range of Mo and $X$ potentials and for the geometries of $\mathrm{Mo}_{6} \mathrm{~S}_{8}, \mathrm{Mo}_{6} \mathrm{Se}_{8}$, and $\mathrm{PbMo}_{6} \mathrm{~S}_{7.5}$ (Figs. 9-11). From the numerical calculations we have obtained approximate, selfconsistent Mo and $\boldsymbol{X}$ potentials.

We have assumed that the $M s$ bands are well below, and the $M p$ bands well above, the region of interest around the Fermi level. The $24 X p$ bands have a total width of about $0.3 \mathrm{Ry}$ and are placed about 0.4 Ry below the center of the 30 Mo $4 d$ bands. The total width of the Mo $d$ bands is $0.5 \mathrm{Ry}$ and this is approximately $1 / \sqrt{3}$ times the $d$-band width in pure Mo metal. Five of the six Mo $s$ bands are well above the top of the Mo $d$ bands, but whether or not the same is true for the lowest Mo $s$-like band, which is strongly repelled by the $X s$ and $X p$ bands, cannot be predicted from our cluster model (Fig. 10).

The Mo $d$ bands are grouped into singly $(A)$, doubly $(E)$, and triply $(T)$ degenerate subbands derived from the individual states of the $\mathrm{Mo}_{6}$ octahedron (Fig. 3). In the binaries, the elongation of the octahedra is sufficiently large that the splitting of the triply degenerate subbands exceeds the subband widths. The relative positions and widths of the Mo $d$ subbands are strongly influenced by the covalent mixing with the $X p$ states (Fig. 9 and Table $\mathrm{V})$. This mixing decreases in the sequence $\mathrm{Mo}_{6} \mathrm{~S}_{8}, \mathrm{Mo}_{6} \mathrm{Se}_{8}, \mathrm{PbMo}_{6} \mathrm{~S}_{7.5}, \mathrm{PbMo}_{6} \mathrm{Se}_{8}$, and $\mathrm{Mo}_{6} \mathrm{Te}_{8}$ (Table IV). The 12 lowest Mo $d$ bands are separated from the 18 highest Mo $d$ bands by a gap of about $0.15 \mathrm{Ry}$.

The Fermi level lies in the $E_{g}$ band below the gap. This band has wave functions of type $x^{2}-y^{2}$ (Fig. 4), its dispersion is given approximately by (26a) and Fig. 6, its state density is shown in Fig. 7 , and its bandwidth is given in Table $\mathrm{V}$ for the various compounds. The $E_{\mathrm{g}}$ bandwidth decreases in the above-mentioned sequence of compounds. 
The $E_{\mathrm{g}}$ states have $85 \%$ Mo $d$ and $14 \%$ intracluster $X p$ character. The important difference between the band structures in the ternaries and the binaries, which we believe is reflected in their physical properties, seems to be that the $E_{g}$ band is distinct in the ternaries but is overlapped with and hybridizes with one or both of the $T_{2 u}$ subbands in the binaries (Fig. 11). A reason for this difference is that the splitting of the $T_{2 u}$ band into $E_{u}$ and $A_{u}$ subbands is small in the ternaries, where the $\mathrm{Mo}_{6}$ octahedra are nearly perfect, and large in the binaries, where the octahedra are elongated by about $4 \%(c / b$ in Fig. 1 and Table I). The position of the $E_{g}$ band above the center of gravity of the $T_{2 u}$ bands depends on the covalent mixing (Fig. 9) and hence on the Mo- $X$ nearest-neighbor distance $\left(b^{\prime} / b\right.$ in Fig. 1 and Table I). The symmetry of the $T_{2 u}$ wave functions is shown in Fig. 5.

We believe that the $E_{\mathrm{g}}$ band is overlapped by a wide Mo $d$ (Mo $s, X p$ )-like $A_{1 g}$ band with wave functions of type $3 z^{2}-r^{2}$, with dispersion $\frac{1}{3}(i+j+k)$ in the notation of (26), and of bandwidth as given in Table V. Owing to the uncertainty in the positions of the Mo $s$-like bands obtained with our cluster model, we cannot decide whether this is the $A_{1 g}$ or the $A_{1 g}^{\prime}$ band (Figs. 10 and 11); nor can we determine the occupancy of this band. Experiments seem to indicate that the broad band is the $A_{1 \mathrm{~g}}^{\prime}$ band and that there are about 0.1 holes in this band. As a consequence, there are about $2.1 E_{\mathrm{g}}$ electrons in $M \mathrm{Mo}_{6} X_{8}$ and about $2.1+2(m-1+x)$ electrons in the $E_{\mathrm{g}}$ band of $M_{m} \mathrm{Mo}_{6} X_{8-x}$ provided that $m \approx 1$ and $x \approx 0$. The estimated Fermi surface of $\mathrm{PbMo}_{6} \mathrm{~S}_{7.5}$ is sketched in Fig. 12.

Our density of states at the Fermi level of 11 states/(spin Mo-atom Ry) for $\mathrm{PbMo}_{6} \mathrm{~S}_{7.5}$ and our theoretical effective exchange-interaction parameter for Mo are in good agreement with the measured paramagnetic susceptibility, yielding an exchange enhancement of 1.8. The measured electronic-specific-heat coefficient is 3.5 times larger than that obtained from our band density of states and this large enhancement may be due to the electron-phonon interaction. The attraction between the $\mathrm{Mo}_{6} X_{8}$ units is dominated by the Mo $d-X p$ co- valency and a calculation of the frequency in one of the rocking modes of these units is in accord with experimental phonon spectra. We find that the average electron-phonon matrix element for the $E_{\text {e }}$ subband is only half the average over the entire Mo $d$ band and depends primarily on the properties of a single octahedron. Its value is seven times smaller than in bcc Mo metal and the electronic contribution to the electron-phonon mass enhancement in $\mathrm{PbMo}_{6} \mathrm{~S}_{7.5}$ is only half the value calculated for Mo metal under the same approximations. The high values observed for the superconducting transition temperature and the electronic-specific-heat coefficient in $\mathrm{PbMo}_{6} \mathrm{~S}_{7.5}$ might therefore be ascribed to low-frequency phonons, such as the rocking modes. We suggest that the high values of the critical fields observed in the ternaries be understood by considering the spin-orbit splitting in the $E_{\mathrm{g}}$ band. In this particular band the spin-orbit coupling is a second-order effect which depends sensitively on the symmetry at the Mo sites and therefore has large spatial fluctuations in non-perfect crystals. We have applied a strong-coupling version of Maki's dirty-limit spin-orbit coupling theory and find good agreement with the measured critical fields.

The model presented here has a number of speculative aspects but, to our knowledge, it does not contradict present experiments and our calculations have been sufficiently explicit and extensive that it should be straightforward to choose alternative routes or add further sophistications. We thus hope to have established a language for future discussions of this most interesting class of materials.

\section{ACKNOWLEDGMENTS}

H. Nohl wishes to thank the Deutsche For schungsgemeinschaft for financial support and the Department of Electrophysics, Technical University of Denmark, for hospitality. The authors acknowledge helpful discussions with Dr. G.B. Jensen, Dr. O. Jepsen, Dr. J. Madsen, and Dr. H. Winter.
${ }^{1}$ R. Chevrel, M. Sergent, and J. Prigent, J. Solid State Chem. $\underline{3}, 515$ (1971). For a review of similar, previously known materials, see H. Schaefer and H. G. Schnering, Angew. Chem. 20, 833 (1964).

${ }^{2} \varnothing$. Fischer, H. Jones, G. Bongi, M. Sergent, and R. Chevrel, J. Phys. C 7, L450 (1974); $\varnothing$. Fischer, A. Treyvand, R. Chevrel, and M. Sergent, Solid State Commun. 17, 721 (1975); $\emptyset$. Fischer, in Proceedings of the 14th International Conference on Low Tempera- ture Physics, Otaniemi, Finland, August 1975, edited by $M$. Krusius and M. Vuorio (North-Holland, Amsterdam, 1975), Vol. 5, p. 172; J. Bolz, G. Crecelius, H. Maletta, and F. Pobell, J. Low Temp. Phys. $\underline{28}$, 61 (1977).

${ }^{3}$ M. Marezio, P. D. Dernier, J. P. Remeika, E. Corenzwit, and B. T. Matthias, Mater. Res. Bull. 8, 657 (1973); O. Bars, J. Guillevic, and D. Grandjean, J. Solid State Chem. $\underline{6}, 48$ (1973); R. Chevrel, M. Ser- 
gent, and J. Prigent, Mater. Res. Bull. 9,1487 (1974).

${ }^{4}$ In the present calculations we assumed that the lattices were cubic, i.e., that $\alpha=90^{\circ}$. For the crystals listed in Table I the real $\alpha$ values are $89.4^{\circ}$ for crystals containing $\mathrm{Pb}$ and $91.5^{\circ}$ for the others.

${ }^{5}$ S. D. Bader, G. S. Knapp, S. K. Sinha, P. Schweiss, and B. Renker, Phys. Rev. Lett. 37, 344 (1976).

${ }^{6}$ O. K. Andersen, Solid State Commun. 13, 133 (1973); Phys. Rev. B 12, 3060 (1975); O. K. Andersen and R. G. Woolley, Mol. Phys. 26, 905 (1973); O. K. Andersen and $\mathrm{O}$. Jepsen, Physica (Utrecht 91B $+\mathrm{C}$, 317 (1977). See also D. G. Pettifor, J. Phys. F 7, 613 (1977); O. Gunnarsson, J. Harris, and R. O. Jones, Phys. Rev. B 15, 3027 (1977).

${ }^{7}$ For a crystal with translation vectors $\overrightarrow{\mathrm{T}}$ and atomic positions $\vec{R}$ in the primitive cell the structure constants are

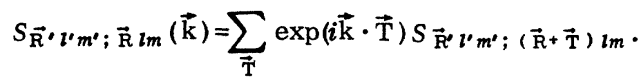

In the present paper we shall generally drop the $\vec{k}$ and thus adopt a notation which does not distinguish between finite clusters and crystals.

${ }^{8}$ The potential parameters, defined by the requirement that (11) and (12) fit (2) in the neighborhood of some arbitrary energy $E_{\nu}$, are related to those defined in Ref. 6 by $C=E_{\nu}+\omega(-l-1), V=E_{\nu}+\omega(l), \mu^{-1}$ $=\frac{1}{2} s_{t}^{3} \Phi^{2}(-l-1), \tau^{-1}=s_{t}^{3} \Phi^{2}(l) /(2 l+3)$, and $\gamma^{-1}$ $=2(2 l+1) \Phi(l) / \Phi(-l-1)$. Moreover, there exists the relation $(2 l+1) s_{t} \Phi(-l-1) \mid \Phi(l)=\omega(-l-1)$ $-\omega(l)$, which is (2.15) in Ref. 6, i.e., $C-V$ $=\left(\mu \gamma s_{t}^{2}\right)^{-1}$, and $\tau=2(2 l+1)^{2}(2 l+3) \mu \gamma^{2}$.

${ }^{9}$ L. Hedin and B. I. Lundqvist, J. Phys. C 4,2064 (1971).

${ }^{10} \mathrm{~J}$. C. Slater and G. F. Koster, Phys. Rev. 94, 1498 (1954).

${ }^{11}$ This conclusion concerns the ratio between the intraand intercluster coupling constants which, in an unhybridized calculation, depends on the decay of the Mo $d$ orbital in the range $\frac{1}{2} R_{\mathrm{MO}-\mathrm{MO}_{0}}^{\mathrm{intra}} \leq r \leq R_{\mathrm{Mo}-\mathrm{MO}_{0}}^{\mathrm{inter}}-\frac{1}{2} R_{\mathrm{Mo}-\mathrm{MO}_{0}}^{\mathrm{inta}}$. For $\mathrm{PbMo}_{6} \mathrm{~S}_{7.5}$ this range is (2.6|3.6) Bohr radii and here our $d$-MTO closely follows the $d$ Slater-type orbital $(d-\mathrm{STO})$ optimized for the cluster $\mathrm{Mo}_{6} \mathrm{Br}_{8}{ }^{4+}$ in Ref. 16. Specifically, the radius at which the MTO and the STO, $r^{l} \exp (-\xi r)$, have the same logarithmic derivative is $r_{0}=(2 l+1) / \xi$, which is $2.8 \mathrm{Bohr}$ radii for the d-STO of Ref. 16. Moreover, for the $d$ bands in transition metals and in transition-metal compounds the unhybridized $d$ bands in the ASA are very good approximations. See, for instance, Ref. 6; O. Jepsen, O. K. Andersen, and A. R. Mackintosh, Phys. Rev. B 12, 3084 (1975); T. Jarlborg and G. Arbman, J. Phys. F $\underline{6}, 189$ (1976); H. Skriver,
Phys. Rev. B 14, 5187 (1976); J. P. Jan and H. Skriver, J. Phys. F 7 , 1719 (1977).

${ }^{12}$ In this section the term "bonding" refers to the coupling between Mo $d$ states. With respect to the Mo $d-X p$ coupling, however, the lowest $d$-like $E_{g}$ and $T_{2 u}$ states will be antibonding.

${ }^{13}$ This includes the $10 \%$ contribution from the hybridization with the $(x z, y z)$ state. The energy unit is $\Delta_{\text {Mod }}$.

${ }^{14}$ The importance of the transition-metal $d$-chalcogen $d$ interaction was pointed out to us by N. J. Doran, G. Wexler, and A. M. Woolley (private communication).

${ }^{15}$ F. A. Cotton and T. E. Haas, Inorg. Chem. 3,10 (1964).

${ }^{16}$ L. J. Guggenberger and A. W. Sleight, Inorg. Chem. 8, 2041 (1969).

${ }^{17}$ L. F. Mattheiss and C. Y. Fong, Phys. Rev. B 15 , 1760 (1977).

${ }^{18}$ R. N. Shelton, A. C. Lawson, and D. C. Johnston, Mater. Res. Bull. 10, 297 (1975).

${ }^{19}$ F. Y. Fradin, G. K. Shenoy, B. D. Dunlap, A. T. Aldred, and C. W. Kimball, Phys. Rev. Lett. 38, 719 (1977).

${ }^{20}$ S. D. Bader, G. S. Knapp, and A. T. Aldred, in Proceedings of the International Conference on Low Lying Lattice Vibrational Modes and Their Relationship to Superconductivity and Ferroelectricity, San Juan, Puerto Rico, December, 1975 (unpublished).

${ }^{21}$ N. Morton, J. G. Booth, and C. F. Woodhead, J. Less Common Met. 34, 125 (1974).

${ }^{22}$ O. K. Andersen, J. Madsen, U. K. Poulsen, O. Jepsen, and J. Kollár, Physica (Utrecht) 86-88B, 249 (1977); U. K. Poulsen, J. Kollár, and O. K. Andersen, J. Phys. F 6, L241 (1976).

${ }^{23}$ W. L. Mc Mïllan, Phys. Rev. 167, 331 (1968).

${ }^{24} \mathrm{D}$. Glötzel and O. K. Andersen (unpublished). See also D. G. Pettifor, J. Phys. F 7, 1009 (1977).

${ }^{25}$ D. A. Papaconstantopoulos, L. L. Boyer, B. M. Klein, A. R. Williams, V. L. Morruzzi, and J. F. Janak, Phys. Rev. B 15, 4221 (1977); W. H. Butler, Phys. Rev. B 15, $52 \overline{67}$ (1977).

${ }^{26}$ G. D. Gaspari and B. L. Gyorffy, Phys. Rev. Lett. 28 , 801 (1972); W. John, J. Phys. F 3, L231 (1973).

${ }^{27}$ B. N. Harmon and S. K. Sinha (unpublished).

${ }^{28}$ M. Peter, J. Ashkenazi, and M. Dacorogna, Helv. Phys. Acta 50, 267 (1977).

${ }^{29} \mathrm{~W}$. Hanke, J. Hafner, and H. Bilz, Phys. Rev. Lett. 37,1560 (1976).

${ }^{30} \mathrm{~K}$. Maki, Phys. Rev. 148, 362 (1966).

${ }^{31} \varnothing$. Fischer (Ref. 2), Fig. 7.

${ }^{32}$ D. Rainer and G. Bergmann, J. Low Temp. Phys. 14, 501 (1974).

${ }^{33} \varnothing$. Fischer (private communication). 